INSTITUTO DE PESQUISAS ENERGÉTICAS E NUCLEARES

Autarquia Associada à Universidade de São Paulo

\title{
MEDIDA DO ESPECTRO DE ENERGIA DOS NÊUTRONS NO NÚCLEO DO REATOR IPEN/MB-01
}

\author{
FERNANDO PRAT GONÇALVES MARTINS
}

Dissertação apresentada como parte dos requisitos para a obtenção do título de Mestre em Ciências na Área de Tecnologia Nuclear - Reatores.

Orientador:

DR. ULYSSES d'UTRA BITELLI

São Paulo

2006 


\section{AGRADECIMENTOS}

Os agradecimentos são direcionados a todos que contribuíram com a elaboração deste trabalho incluindo aqueles que porventura, não estejam relacionados aqui.

Ao Dr. Ulysses d’Utra Bitelli, pela amizade, compreensão, apoio, incentivo e pelas constantes e inestimáveis orientações que foram imprescindíveis para a conclusão deste trabalho.

A minha mulher Andreia, pelo carinho, compreensão e por me dar forças nas horas mais difíceis, clareza sempre que necessário e por estar sempre ao meu lado, me apoiando e motivando. E por ultimo, mas não menos importante, agradeço a meu filho Caique pela paciência e carinho.

Aos meus pais, José Carlos D.G. Martins e Elizabeth Prat E. Martins, principais responsáveis pela minha formação, pelo carinho, apoio e incentivos constantes, que me motivaram para a realização deste trabalho.

A minha irmã, Samantha Eslava G. Martins, pelo constante apoio e incentivo.

A colega Rosangela Ramalho Cacure, pelo auxílio na obtenção de alguns dados para a geração de resultados.

Ao colega César Luiz Veneziani pelo auxílio na obtenção e formatação de gráficos e figuras.

Ao colega Lucas Batista Gonçalves pelo fundamental apoio no cálculo dos fatores de auto-blindagem.

Aos Drs. Aucyone Augusto da Silva e Wilson José Vieira pelas inestimáveis sugestões no aprimoramento deste trabalho.

A todos os colegas do Reator IPEN/MB-01, que de alguma forma contribuíram para a conclusão deste trabalho. 
A todos os professores que me forneceram subsídios fundamentais para a elaboração desta dissertação.

A Deus. 


\title{
MEDIDA DO ESPECTRO DE ENERGIA DOS NÊUTRONS NO NÚCLEO DO REATOR IPEN/MB-01
}

\section{Fernando Prat Gonçalves Martins}

\begin{abstract}
RESUMO
Este trabalho apresenta a medida do espectro de energia dos nêutrons no núcleo do Reator IPEN/MB-01. Para tal, foram inseridos detectores de ativação na forma de diminutas folhas metálicas no núcleo do reator, na região moderadora, utilizando um dispositivo articulado que permite que as folhas fiquem posicionadas na posição central do núcleo do reator, garantindo que todas as folhas sejam irradiadas na mesma posição. Os detectores de ativação foram selecionados de forma a cobrir grande parte do espectro de energia dos nêutrons no Reator, para tanto foram utilizadas folhas de $\mathrm{Au}^{197}, \mathrm{Mg}^{24}, \mathrm{Ti}^{48}$, $\mathrm{In}^{115}, \mathrm{Sc}^{45}$ entre outras. Após a irradiação os detectores de ativação foram submetidos a espectrometria gama num sistema de contagem de Germânio hiper-puro, o que possibilitou a obtenção da atividade de saturação por núcleo alvo, um dos principais dados de entrada do código computacional de desdobramento de espectro "unfolding" SANDBP, que através de um processo iterativo, ajusta o espectro que melhor se adequa ao conjunto de dados de entrada do código, composto principalmente pelas taxas de reação por núcleo alvo obtidas experimentalmente e um espectro inicial de entrada, neste caso obtido à nível celular pelo código Hammer-Technion para a célula representativa do núcleo do Reator, fornecendo assim a solução do espectro.
\end{abstract}




\title{
MEDIDA DO ESPECTRO DE ENERGIA DOS NÊUTRONS NO NÚCLEO DO REATOR IPEN/MB-01
}

\section{Fernando Prat Gonçalves Martins}

\begin{abstract}
This work presents the neutron spectrum measurements in the Reactor IPEN/MB01 using very thin activation detectors in the metallic form, in reactor core, in moderator region. An articulated device allows that the foils are inserted in the central position of reactor core, ensuring that all the foils are irradiated in the same position. The activation detectors of different materials such $\mathrm{Au}^{197}, \mathrm{Mg}^{24}, \mathrm{Ti}^{48}, \mathrm{In}^{115}, \mathrm{Sc}^{45}$ and others, were selected to cover a large range of neutron spectrum. After the irradiation, the activation detectors were submitted to a spectrometry gamma by using a system of counting with high purity Germanium, to obtain the saturation activity per target nuclide. The saturation activity is one of the main data of input of unfolding code SANDBP, that through an iterative adjustment, modify the spectrum that better agree with the dataset of code input, composition mainly for measure reaction rate per target nuclide and a initial input spectrum, calculated for Hammer-Technion code, supplying a solution spectrum.
\end{abstract}




\section{SUMÁRIO}

1. INTRODUÇÃ

1.1. Métodos de Detecção de Nêutrons e Espectrometria..........................................2

1.2. Métodos de Medida de Espectro de Nêutrons.......................................................3

2. MEDIDA DE ESPECTRO PELO MÉTODO DE ATIVAÇÃO..................................5

2.1. Princípio do Método de Ativação por Nêutrons.....................................................5

2.2. Espectrometria de Nêutrons Pela Ativação de Múltiplos Detectores....................9

2.3. Espectro de Nêutrons Térmicos.......................................................................14

2.4. Espectro de Nêutrons Rápidos........................................................................16

2.5. Espectro de Nêutrons Epitérmicos.....................................................................19

2.6. Medidas de Fluxo Térmico e Epitérmico............................................................ 20

2.7. Medidas de Fluxo Rápido.............................................................................23

2.8. Fatores de Correção Para Perturbação do Fluxo de Nêutrons.............................25

3. MATERIAIS E MÉTODOS EXPERIMENTAIS................................................29

3.1. Seleção das Folhas de Ativação.........................................................................29

3.2. Características das Folhas de Ativação............................................................30

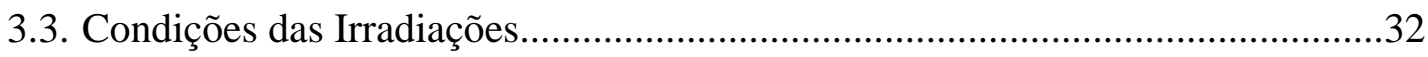

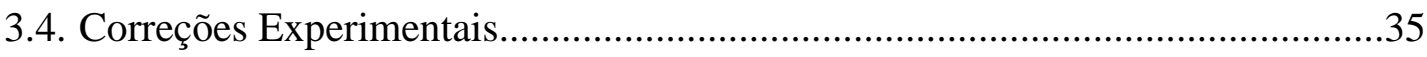

3.4.1. Fator de Normalização............................................................................35

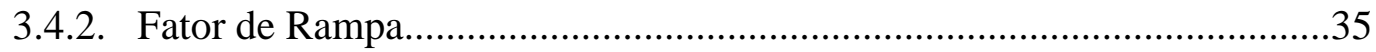

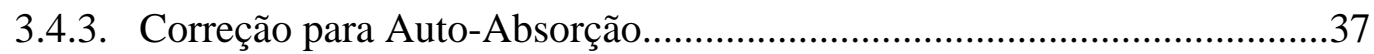

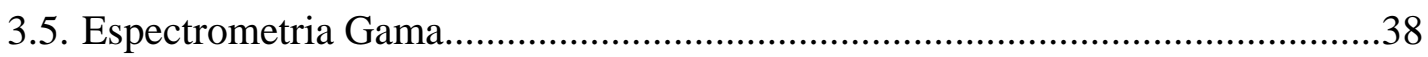

3.5.1. Cálculo da Eficiência do Sistema de Detecção HPGe...............................38

3.5.2. Espectrometria Gama das Folhas Irradiadas...........................................41

3.5.3. Determinação dos Parâmetros de Entrada do Código SANDBP...............41 
3.6. Análise das Incertezas Experimentais. .46

4. UTILIZAÇÃO DO CÓDIGO SANDBP 49

4.1. Introdução. .49

4.2. Procedimento Matemático. .49

5. RESULTADOS EXPERIMENTAIS .54

6. COMPARAÇÃO DOS VALORES MEDIDOS E CALCULADOS. .66

7. CONCLUSÕES .71

ANEXO A - O REATOR NUCLEAR IPEN/MB-01......................................73

ANEXO B - CONJUNTO DE DADOS DE ENTRADA DO CÓDIGO SANDBP (INPUT). .82

ANEXO C - SAIIDA DO CÓDIGO SANDBP. .84

ANEXO D - FLUXO DE NÊUTRONS DO REATOR IPEN/MB-01 E SUAS

INCERTEZAS.

ANEXO E - CURVAS DE SEÇÃO DE CHOQUE DOS MATERIAIS

UTILIZADOS COMO DETECTORES DE ATIVAÇÃO. .91 ANEXO F - CÁLCULO DO FATOR DE AUTO-BLINDAGEM PARA A FOLHA DE OURO NUA E COBERTA COM CÁDMIO PELO MÉTODO DE MONTE-CARLO. .96

REFERÊNCIAS BIBLIOGRÁFICAS. 101 


\section{INTRODUÇÃO}

A determinação da distribuição energética dos nêutrons, ou seja, do espectro de energia dos nêutrons no núcleo de um reator nuclear é de fundamental importância para muitos propósitos de interesse da Física, Engenharia, projeto, segurança e operação de centrais nucleares. Em reatores de potência o espectro de nêutrons a que estão expostos os vasos de pressão, acabam definindo o tempo útil de utilização da planta devido a sua fragilização aos danos por radiação.

O espectro de nêutrons em uma determinada posição interna ao núcleo de um reator pode ser estimado através de cálculos a partir da equação de transporte de nêutrons, que descreve matematicamente o comportamento neutrônico nesta posição. Todavia, as soluções da equação de transporte de nêutrons geralmente envolvem simplificações de cálculo, que acrescidas à imprecisão de dados nucleares, heterogeneidade do reator e dificuldade de modelagens adequadas, levam a uma solução aproximada. Por estas razões, o resultado oriundo do cálculo pode ser, muitas vezes, considerado apenas como uma informação preliminar, que pode ser aperfeiçoada utilizando os resultados experimentais. Sendo assim, é fundamental que se façam medidas experimentais para validação e, se necessário, adequação do cálculo à realidade física do problema.

O espectro de energia dos nêutrons é, por conveniência, dividido em três regiões ou grupos de energia: A região térmica, constituída por nêutrons em equilíbrio térmico com o moderador, a região rápida ou de fissão, na qual os nêutrons de físsão são produzidos, e a região intermediária onde os nêutrons se encontram em processo de moderação de sua energia.

A distribuição energética dos nêutrons no núcleo do reator, bem como a distribuição espacial em grupos de energia térmico, epitérmico ou rápido, é de fundamental importância, pois do seu conhecimento pode-se aferir com razoável precisão uma série de parâmetros neutrônicos como taxas de reação, queima de combustível, reatividade, etc. 


\subsection{Métodos de Detecção de Nêutrons e Espectrometria}

Pelo fato dos nêutrons não possuírem carga elétrica, eles não ionizam os materiais do meio em que interagem; portanto, a detecção dos nêutrons é feita indiretamente através de reações nucleares induzidas por essas partículas. A detecção dos nêutrons térmicos e epitérmicos pode ser feita com base nos seguintes procedimentos:

- Detecção instantânea da carga dos produtos decorrentes de uma reação dos nêutrons com elementos leves. A mais importante reação desta categoria é a reação ${ }^{10} \mathrm{~B}(\mathrm{n}, \alpha)^{7} \mathrm{Li}$; neste caso, a seção de choque da reação tem um comportamento $1 / \mathrm{v}$, onde $\mathrm{v}$ é a velocidade do nêutron, sendo muito utilizada o gás $\left(\mathrm{BF}_{3}\right)$ em detectores proporcionais;

- Detecção dos produtos pela reação de captura radioativa de nêutrons $(n, \gamma)$ ou de fissões induzidas; Os elementos que possuem alta seção de choque para nêutrons térmicos são: $\mathrm{U}^{233}, \mathrm{U}^{235}$ e $\mathrm{Pu}^{239}$. A detecção dos produtos da reação pode ocorrer com detectores termoluminescentes.

A detecção dos nêutrons rápidos geralmente é feita através dos seguintes procedimentos:

- Detecção dos prótons de recuo produzidos pelo espalhamento elástico dos nêutrons rápidos nos materiais hidrogenados. Esta forma de iteração é usada em câmaras de ionização e emulsões nucleares;

- Detecção instantânea da carga dos produtos da reação para elementos leves, tais como ${ }^{3} \mathrm{He}(\mathrm{n}, \mathrm{p})^{3} \mathrm{H}$ e ${ }^{6} \mathrm{Li}(\mathrm{n}, \alpha){ }^{3} \mathrm{H}$;

- Detecção dos produtos de reações de limiar, como $(n, p),(n, \alpha)$,etc..., e fissão nuclear induzida por nêutrons rápidos. Os materiais que apresentam alta seção de choque para nêutrons rápidos, e consequientemente, possuem grande probabilidade de fissionar com a incidência de nêutrons rápidos são: $\mathrm{Th}^{232}, \mathrm{~Np}^{237}$ e $\mathrm{U}^{238}$. 


\subsection{Métodos de Medida do Espectro de Nêutrons}

Diversos métodos foram desenvolvidos para medida do espectro de nêutrons; Cada método apresenta algumas vantagens e desvantagens, e sua aplicação depende principalmente da faixa de energia dos nêutrons.

O espectro de energia de um reator nuclear pode ser dividido em três regiões: térmica, intermediária e rápida, respectivamente em ordem crescente de energia. $\mathrm{O}$ espectro na região térmica leva em conta todos os nêutrons com energia inferior a $0,5 \mathrm{eV}$ e pode ser aproximado por uma maxwelliana. $\mathrm{O}$ espectro intermediário tem a forma $1 / \mathrm{E}$ e engloba nêutrons de energias compreendidas entre $0,5 \mathrm{eV}$ e 0,5 $\mathrm{MeV}$. $\mathrm{O}$ espectro de nêutrons rápidos compreende nêutrons com energia superior a $0,5 \mathrm{MeV}$ e pode ser representado pelo espectro de Watt $^{\prime 20 /}$.

Em reatores o espectro pode ser medido "in-core" por folhas de ativação e "out-ofcore" com detectores do tipo NE-213, bem como seletores de velocidade de nêutrons (“shopper”) em canais de irradiação (“beam-holes”) de reatores de pesquisa.

Para medida do espectro de nêutrons "in-core" o método de ativação por múltiplos detectores é um dos mais importantes e utilizados. Este método utiliza os resultados obtidos pela ativação de folhas irradiadas em determinada posição do reator, para a obtenção do espectro de nêutrons. Este procedimento requer uma informação inicial da forma do espectro de entrada, que será ajustado pelo resultado das medidas experimentais. Este método é um dos mais aceitáveis para medida do espectro "in-core", devida a não interferência do campo gama do núcleo na resposta dos detectores, bem como o pequeno tamanho dos detectores (folhas de ativação).Uma das dificuldades apresentadas por este método é a baixa disponibilidade de detectores de ativação para medida na faixa de energia entre $100 \mathrm{kev}$ e $1 \mathrm{Mev}{ }^{\text {/49/. }}$ Outro problema é a perturbação do fluxo durante a irradiação; o uso de detectores de ativação de materiais com alta seção de choque macroscópica de absorção permite a medida de nêutrons, mas provoca distorções no espectro de nêutrons de interesse. Por outro lado, materiais com baixa seção de choque macroscópica de absorção irão reduzir esta distorção, mas em geral, necessitam de um longo tempo de irradiação. 
Outras técnicas utilizadas para medida de espectro de energia dos nêutrons, como tempo de vôo e prótons de recuo são descritas a seguir:

A técnica do Tempo de Vôo consiste em determinar a energia do nêutron e sua respectiva energia cinética, através do tempo de percurso e caminho de vôo (distância percorrida) pelo nêutron. Ou seja, é necessário conhecer o instante em que o nêutron deixa o ponto inicial e o instante em que alcança o final do percurso, razão pela qual se utiliza dois detectores de nêutrons, um no início e outro no fim do percurso. A principal vantagem desta técnica é a de possibilitar medidas do espectro em regiões de baixa energia, onde outras técnicas muitas vezes não alcançam. A desvantagem está no grande e caro aparato experimental (grandes distâncias para obter boa precisão na medição de nêutrons de alta energia).

A técnica de emulsão nuclear fornece bons resultados na região compreendida entre as energias de $350 \mathrm{keV}$ e $10 \mathrm{MeV}$, mas sua aplicação é restrita ao núcleo de reatores.

A técnica de prótons de recuo se baseia na propriedade dos nêutrons rápidos, ao interagirem com materiais hidrogenados e arrancarem prótons denominados "de recuo". Com a utilização de detectores de cintilação do tipo orgânico, sólido ou líquido, é possível medir os prótons de recuo da interação nêutron-material do cintilador. Estes prótons depositam toda sua energia no cintilador, provocando ionizações ao longo de sua trajetória, que são proporcionais à energia do próton e conseqüentemente do nêutron. ${ }^{/ 3 /}$.

O método de prótons de recuo com a utilização de detectores do tipo contador proporcional é apropriado para a medida do espectro de nêutrons, no entanto, devido às dimensões físicas dos contadores, sua aplicação dentro do núcleo de reatores torna-se muito difícil.

Uma descrição matemática do método de ativação (método utilizado neste trabalho) pode ser vista no Capítulo 2. 


\section{MEDIDA DO ESPECTRO DE ENERGIA PELO MÉTODO DE ATIVAÇÃO}

O fluxo de nêutrons e a medida do espectro em reatores nucleares são usualmente feitos pelo método de ativação, através da irradiação de materiais no campo de nêutrons a ser investigado. A atividade destes materiais (detectores de ativação) será proporcional ao fluxo de nêutrons, a que os mesmos estiveram expostos durante a sua irradiação.

\subsection{Princípio do Método de Ativação por Nêutrons}

Submetido um detector de ativação a um campo neutrônico em estado estacionário durante um tempo de irradiação $t$, a taxa de produção de átomos radioativos é dada por ${ }^{15 /}$ :

$$
\frac{d N}{d t}=N \overline{\boldsymbol{\sigma}}_{a c t} \Phi-N \overline{\boldsymbol{\sigma}}_{a} \Phi-N \lambda
$$

Sendo,
$N \quad$ o número de átomos do radionuclídeo formado,
$\overline{\boldsymbol{\sigma}}_{a c t} \quad$ seção de choque média de ativação,
$\overline{\boldsymbol{\sigma}}_{a} \quad$ seção de choque média de absorção,
$\lambda \quad$ a constante de decaimento do radionuclídeo formado,
$\Phi \quad$ o fluxo de nêutrons.

Na equação (2.1), o primeiro termo após a igualdade refere-se à taxa de produção do radionuclídeo, o segundo termo refere-se a taxa de eliminação por absorção do radionuclídeo e o último termo refere-se à taxa de eliminação por decaimento radioativo.

De forma análoga, a taxa de consumo total dos átomos alvo conhecida como queima “ Burn-up" é dada por:

$$
\frac{d N}{d t}=-N \overline{\boldsymbol{\sigma}}_{t} \Phi
$$


Onde $\bar{\sigma}_{t}$ é a seção de choque média total no espectro, que leva em conta todas as possíveis reações de consumo dos átomos alvos. A solução da equação (2.2) é dada por:

$$
N=N_{0} \cdot e^{-\bar{\sigma}_{t} t}
$$

Sendo $N_{0}$ o número de átomos alvo no início da irradiação. Substituindo (2.3) em (2.1), têm-se que:

$$
\frac{d N}{d t}+N\left(\lambda+\bar{\sigma}_{a} \Phi\right)=N_{0} \overline{\boldsymbol{\sigma}}_{a t v} \Phi \cdot e^{-\bar{\sigma}_{t} \Phi t}
$$

Resolvendo a equação (2.4) pela técnica do fator integrante, que consiste em multiplicar ambos os lados da igualdade da equação por $e^{\left(\lambda+\bar{\sigma}_{a} \Phi\right) t}$, obtém-se a seguinte igualdade:

$$
\frac{d}{d t}\left[N e^{\left(\lambda+\bar{\sigma}_{a} \Phi\right) t}\right]=N_{0} \bar{\sigma}_{a} \Phi e^{-\left[\left(\lambda+\bar{\sigma}_{a} \Phi\right)+\bar{\sigma}_{t} \Phi\right] t}
$$

Integrando a equação (2.5) entre 0 e t, e considerando que $\bar{\sigma}_{a} \Phi<<\lambda$ e $\quad \bar{\sigma}_{t} \Phi<<$ $\lambda$, obtém-se o número de átomos radioativos $n$ formados no tempo de irradiação $t$ :

$$
N=\frac{\overline{\boldsymbol{\sigma}}_{a c t} N^{\prime} V\left(1-e^{-\lambda t}\right)}{\lambda}
$$

Onde $N$ ' é o número de átomos alvo por $\mathrm{cm}^{3}$ do detector irradiado e $V$ é o seu volume. Sendo $A_{0}=n \cdot \lambda$ a atividade inicial do detector ao término de sua irradiação $\left(t=t_{0}\right)$ e $\overline{\boldsymbol{\sigma}}_{a c t} N^{\prime}=\Sigma_{a c t}$ a seção de choque macroscópica média de ativação, pode-se reescrever a equação (2.6) da seguinte forma:

$$
A_{0}=\Sigma_{a c t} \Phi V\left(1-e^{-\lambda t}\right)
$$


Observando a equação (2.7) verifica-se que ao irradiarmos um detector durante um tempo grande (maior que a meia-vida do nuclídeo formado), o termo $e^{-\lambda t}$ tende a zero, de tal forma que $A_{0}$ tenderia a um valor máximo. Este valor máximo é conhecido como atividade de saturação do detector $A^{\infty}$.Para quantificarmos o que foi dito, tempos de irradiação equivalentes a 4 vezes a meia-vida do nuclídeo formado são suficientes para induzir uma atividade $6 \%$ inferior à atividade de saturação ${ }^{122}$. Sendo assim, pode-se reescrever a equação anterior da seguinte forma:

$$
A_{0}=A^{\infty}\left(1-e^{-\lambda t}\right)
$$

Comparando as equações (2.7) e (2.8) conclui-se que:

$$
A^{\infty}=\Sigma_{a c t} \Phi . V
$$

Onde o termo $\Sigma_{a c t} \Phi V$ é numericamente igual ao valor da Taxa de Reação nuclear, que como pode ser visto na equação (2.9) é igual a atividade de saturação.

Na figura (2.1) pode-se visualizar a variação da atividade $A$ do detector em função do tempo $t$; até o tempo $t=t_{0}$ o detector é irradiado, instante no qual é retirado do reator e inicia-se o processo de decaimento radioativo; entre os instantes $t_{1}$ e $t_{2}$ a atividade absoluta do detector é determinada experimentalmente num sistema de detecção calibrado em energia e que apresente uma eficiência global de contagem $\varepsilon$. 


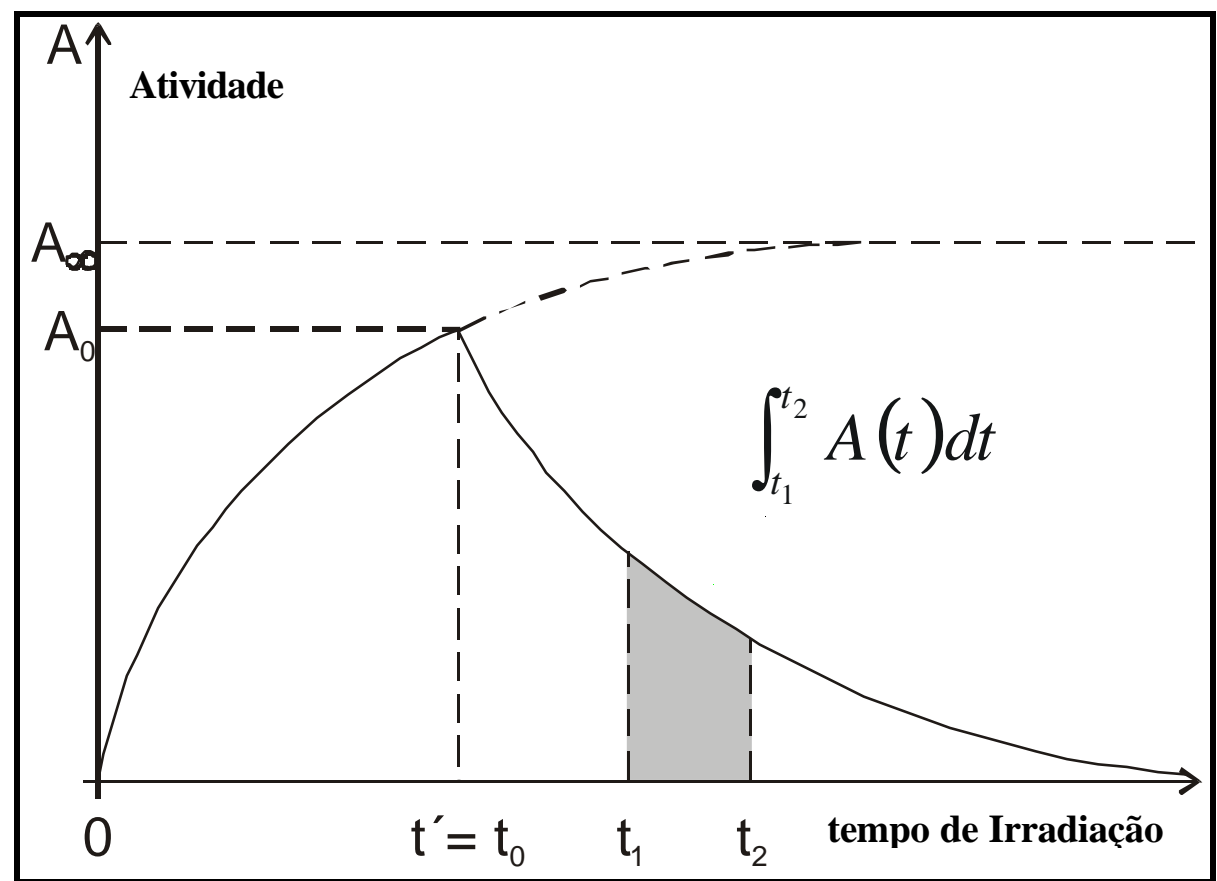

Figura 2.1. Variação da atividade do detector em função do tempo de irradiação.

Assim, a contagem $C$ obtida para um detector cuja atividade é medida entre os instantes $t_{1}$ e $t_{2}$ é dada pela seguinte expressão:

$$
C=\varepsilon \int_{t_{1}}^{t_{2}} A_{0} e^{-\lambda t} d t+B G
$$

Sendo,

$\varepsilon \quad$ eficiência global de contagens do sistema de detecção,

I probabilidade de emissão gama do fotopico contado,

$A_{0} \quad$ atividade ao término da irradiação,

$B G$ radiação de fundo (Background).

Resolvendo a equação (2.10) e definindo $t_{e}$ como sendo o tempo de espera para contagem dado por $t_{1}-t_{0}$ e o tempo de contagem $t_{c}=t_{2}-t_{1}$, chega-se a seguinte expressão: 


$$
C=\frac{\varepsilon \cdot I \cdot A_{0}}{\lambda} e^{-\lambda t_{e}}\left(1-e^{-\lambda t_{c}}\right)+B G
$$

Por fim, substituindo a equação (2.8) em (2.1), obtém-se a atividade de saturação, dada por:

$$
A^{\infty}=\frac{\lambda(C-B G) e^{\lambda t_{e}}}{\varepsilon \cdot I\left(1-e^{-\lambda t}\right)\left(1-e^{-\lambda t_{c}}\right)}
$$

Através da equação (2.12) pode-se obter experimentalmente a taxa de reação e fazer uma comparação com o valor obtido através de uma determinada metodologia de cálculo e sua biblioteca de dados nucleares associada. Podem ser utilizados no cálculo as teorias de difusão, transporte de nêutrons e o método de Monte Carlo, validando assim metodologias de cálculo e bibliotecas de dados nucleares. Isto faz com que esta equação seja de extrema importância para a área experimental de Física de Reatores.

\subsection{Espectrometria de nêutrons pela ativação de múltiplos detectores}

Na década de 60, mais precisamente em 1967, McElroy, Berg e outros desenvolveram um programa computacional conhecido pelo nome de SAND /27/ (“Spectrum Analysis by Neutron Detectors). Tal programa realiza o desdobramento do espectro a partir de dados experimentais, fornecidos pela ativação de diferentes folhas num mesmo campo neutrônico.

Matematicamente, o problema da técnica de desdobramento de espectro pela ativação de várias folhas consiste em resolver um conjunto de equações acopladas do $\operatorname{tipo}^{/ 49 /}$ :

$$
A_{i}=\int_{0}^{\infty} \sigma_{i}(E) \cdot \Phi(E) d E
$$

Sendo, 
$A_{i} \quad$ a atividade de saturação por núcleo alvo determinada experimentalmente para a i-ésima folha,

$\sigma_{i}(E)$ seção de choque em função da energia,

$\Phi(E) \quad$ a densidade de fluxo de nêutrons por unidades de intervalo de energia (espectro de nêutrons) .

A solução da equação (2.13) usualmente é obtida introduzindo a estrutura de grupo na energia dos nêutrons, o que resulta no seguinte sistema de equações lineares:

$$
A_{i}=\sum_{j=1}^{m} \sigma_{i, j} \Phi_{j}
$$

Sendo,
$A_{i} \quad$ a atividade de saturação para a i-ésima reação,
n o número de reações, $\mathrm{i}=1, \ldots, \mathrm{n}$,
m o número de grupos de energia,
$\sigma_{i, j} \quad$ a seção de choque média do j-ésimo grupo para a i-ésima reação,
$\Phi_{j} \quad$ o fluxo de nêutrons do j-ésimo grupo.

$\mathrm{Na}$ equação acima, o número de incógnitas (valores de fluxo) é geralmente muito maior do que o número de equações (determinado pela quantidade de detectores irradiados), assim para encontrar uma única solução é necessário uma informação inicial do espectro de nêutrons (espectro de entrada), onde este espectro deve possuir a provável forma do espectro de nêutrons na posição de interesse.

O procedimento consiste em irradiar o conjunto de detectores de ativação na posição onde deseja-se obter o espectro de energia dos nêutrons, e posterior medida dos valores absolutos de atividade de saturação por núcleo alvo das folhas.

Os valores destas atividades e os dados das seções de choque são então utilizados para ajustar o espectro de entrada. 
Muitos métodos foram desenvolvidos para resolver o sistema de equações lineares dado pela equação (2.14), usando diferentes critérios de aceitabilidade para a solução do espectro. Para tal, grandes esforços foram direcionados para desenvolver e testar diversos códigos computacionais de ajuste de espectro com algoritmos baseados nesses métodos $^{/ 48,53 /}$.

O código computacional SPECTRA / 17, 18 /, utiliza um método de perturbação que modifica o espectro de entrada até que ele concorde com os dados da ativação. A solução é encontrada usando o método dos mínimos quadrados, que minimiza a diferença entre os valores das atividades medidas e calculadas dos detectores de ativação, através da seguinte equação:

$$
S^{k}=\sum_{i=1}^{n} W_{i}\left(\frac{A_{i}-A_{i}^{k}}{A_{i}}\right)^{2}+\sum_{j=1}^{m}\left(\frac{\Phi_{j}^{k}-\Phi_{j}^{k-1}}{\Phi_{j}^{k-1}}\right)^{2}
$$

Onde,

$$
A_{i}^{k}=\sum_{j=1}^{m} \sigma_{i, j} \Phi_{j}^{k}
$$

Sendo,

$$
\begin{array}{ll}
W_{i} & \text { uma função peso; } \\
k & \text { índice que indica saída(output) na k-ésima iteração. }
\end{array}
$$

A solução para o espectro é então obtida por um processo iterativo usando o resultado da expressão da minimização desta diferença. O processo de iteração é interrompido quando o erro médio da atividade dos detectores de ativação torna-se menor que o valor da entrada;

O erro médio da atividade (ERRE) para a k-ésima iteração é calculado da seguinte forma:

$$
\operatorname{ERRE}^{k}=\frac{1}{n} \sum_{i=1}^{n}\left(\frac{A_{i}-A_{i}^{k}}{A_{i}}\right)
$$


O código SPECTRA foi desenvolvido após outro código computacional, conhecido como RFSP $^{/ 11,12 /}$. Esses 2 códigos diferem um do outro principalmente pelas relações matemáticas utilizadas no método de solução, onde o método do código RFSP inclui peso nas incertezas. O método de solução destes dois códigos requer inversão da matriz, e é possível obter o espectro em no máximo de 100 grupos energia.

Outro código de ajuste de espectro conhecido como CRYSTAL BALL ${ }^{121 /}$. utiliza um diferente método de ajuste. Este método é baseado na aproximação do operador delta de Dirac pela combinação linear dos operadores integrais. A solução do espectro $\Phi_{g}(E)$ é necessária para minimizar a seguinte expressão:

$$
S^{2}=\int_{0}^{\infty}\left(\frac{d}{d E} \frac{\Phi_{g}(E)}{\Phi_{i}(E)}\right)^{2} W(E) d E
$$

Onde,

$\Phi_{i}(E)$ é o espectro de entrada;

$W(E)$ é uma função peso.

O código CRYSTALL BALL utiliza um processo iterativo, que é interrompido se o Desvio Médio Relativo (ADR) for menor que um valor especificado. O Desvio Médio Relativo para k-ésima iteração é dado pela seguinte expressão:

$$
A R D^{k}=\frac{1}{n} \sum_{i=1}^{n} \frac{\left(A_{i}-A_{i}^{k}\right)^{2}}{\operatorname{Var}\left(A_{i}\right)}
$$

A solução do espectro obtida usando este método não é sensível ao espectro de entrada, exceto nas faixas de energia que são pouco cobertas pela resposta dos detectores. É importante notar que este método utiliza a variância das taxas de reação medidas como um fator peso na expressão (2.18), o que consiste em uma vantagem no processo iterativo.

Um terceiro código de ajuste de espectro de nêutrons é o código STAY'SL ${ }^{132 /}$. Este código utiliza o princípio dos mínimos quadrados generalizados, assumindo que as taxas de reação, as seções de choque de grupo e as densidades de fluxo de grupo são variáveis 
aleatórias, e suas incertezas associadas seguem uma distribuição normal. Para tal é utilizada a seguinte expressão quadrática de minimização:

$$
X^{2}(\Phi, \sigma, A)=\frac{\left(\Phi^{0}-\Phi\right)^{t}\left(\Phi^{0}-\Phi\right)}{V\left(\Phi^{0}\right)}+\frac{\left(\sigma^{0}-\sigma\right)^{t}\left(\sigma^{0}-\sigma\right)}{V\left(\sigma^{0}\right)}+\frac{\left(A^{0}-A\right)^{t}\left(A^{0}-A\right)}{V\left(A^{0}\right)}
$$

Sendo,

$\Phi \quad$ vetor de saída (solução) dos valores de fluxo de grupo;

$\sigma \quad$ matriz de saída das seções de choque de grupo para todas reações;

A vetor de saída das taxas de reação baseadas em $\Phi e \sigma$,

$V($ ) a matriz covariância dos parâmetros informados dentro dos parênteses;

0 denota os valores de entrada;

$t \quad$ denota matriz transposta.

O método dos mínimos quadrados garante desta forma uma solução verdadeira, utilizando um conjunto de dados de entrada consistente, embora necessite da informação da covariância para todos os parâmetros de entrada. Assim, a principal desvantagem deste código é a preparação do conjunto de dados de entrada.

O código de ajuste de espectro de nêutrons SANDII ${ }^{127 /}$ é outro código de ajuste conveniente para ser utilizado. Embora este código não forneça uma rigorosa solução "best fit" que minimiza uma expressão de mínimos quadrados, cada passo de iteração do código corresponde à um processo de mínimos quadrados para o logaritmo dos valores da atividade e densidade de fluxo.

O código SANDBP, código este utilizado neste trabalho é uma versão aperfeiçoada do código SANDII. Uma descrição detalhada do código SANDBP é dada no capítulo 4.

Resultados obtidos em congressos de códigos de ajuste, indicam o uso de códigos baseados no princípio dos mínimos quadrados generalizados ${ }^{/ 43,44 /}$. 


\subsection{Espectro de Nêutrons Térmicos}

Quando os nêutrons atingem um estado de equilíbrio térmico com os átomos do moderador, suas energias passam a ser determinadas pela distribuição de energia térmica dos átomos do moderador e o espectro de energia dos nêutrons torna-se uma distribuição maxwelliana na temperatura $T$ do material moderador. Desta forma, tanto o fluxo de nêutrons, como a densidade de nêutrons são expressos como função da velocidade dos nêutrons ou da energia dos nêutrons.

Embora a distribuição maxwelliana seja uma boa aproximação do espectro de nêutrons térmicos em algumas posições do reator, os nêutrons só atingem de fato o equilíbrio térmico com o meio em regiões onde a absorção seja pequena, como por exemplo, uma coluna de grafite.

A densidade de nêutrons como função da velocidade $n(v)$ é dada por ${ }^{120 /}$ :

$$
n(v)=\frac{4}{\sqrt{\pi}}\left(\frac{m}{2 k T}\right)^{\frac{3}{2}} v^{2} e^{\left(\frac{-m v^{2}}{2 k T}\right)}
$$

O fluxo de nêutrons em função da energia $\Phi(E)$ é dado por:

$$
\Phi(E)=\frac{E}{(K T)^{2}} e^{\left(\frac{-E}{K T}\right)}
$$

As equações (2.20) e (2.21) são normalizadas por unidade de área, como pode ser visto nas figuras 2.2 e 2.3 : 


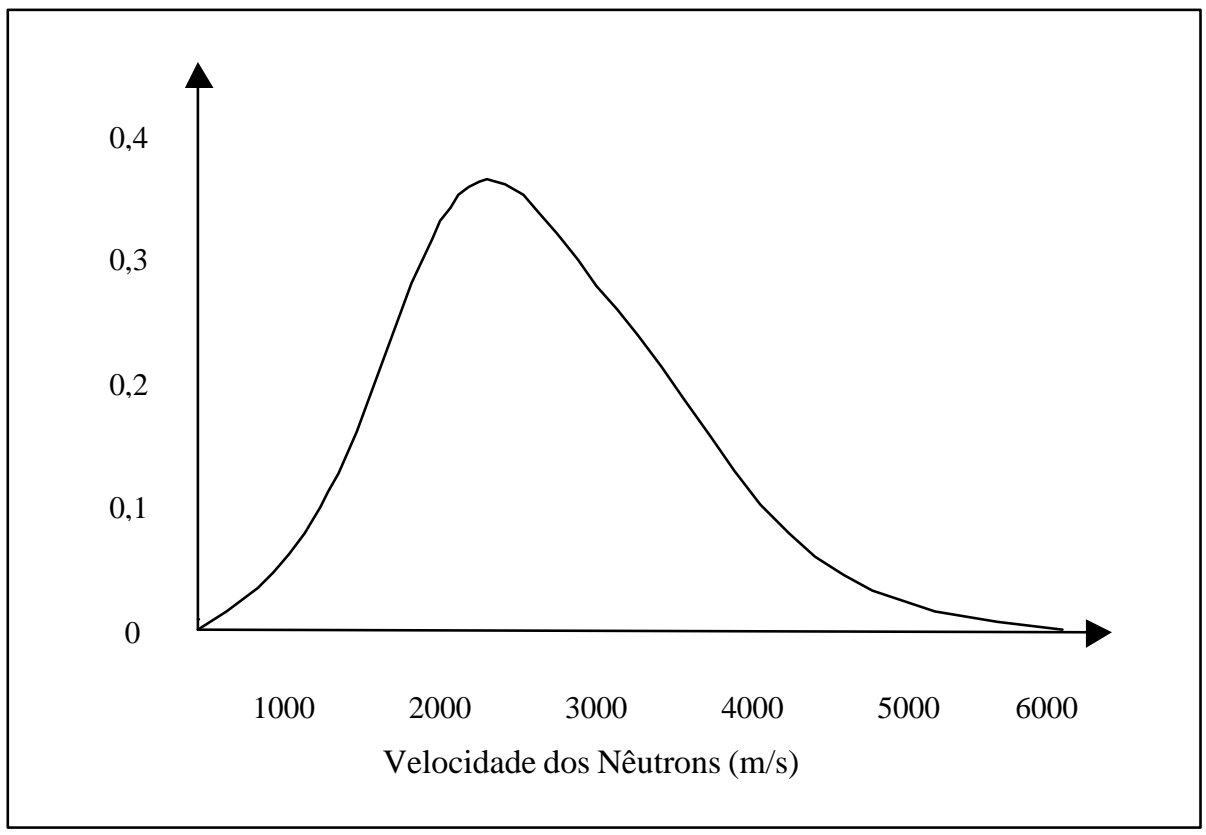

Figura 2.2. Distribuição de Velocidade - Maxwelliana

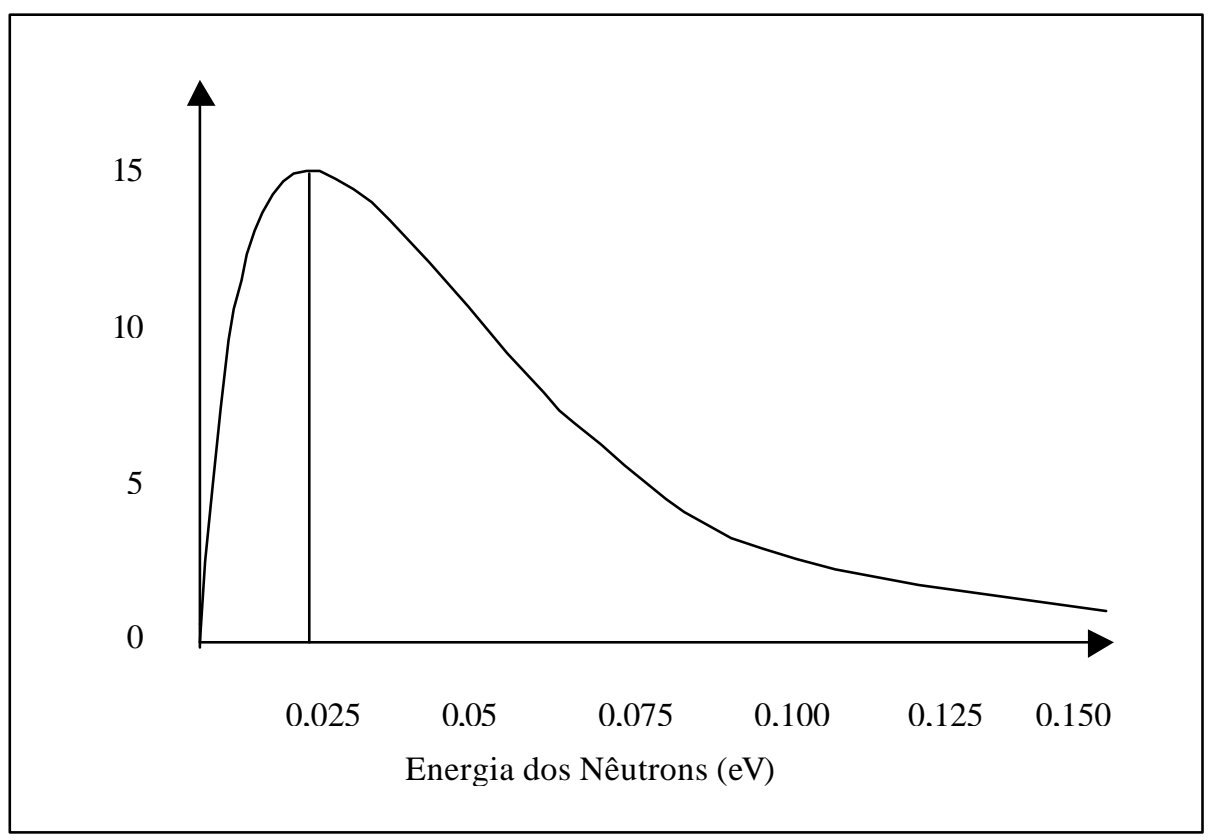

Figura 2.3. Distribuição do Fluxo - Maxwelliana 
Da equação (2.20) têm-se que a velocidade na energia mais provável é dada por:

$$
v_{0}=\left(\frac{2 K T}{m}\right)^{\frac{1}{2}}
$$

o que corresponde à energia de $\frac{m v_{0}^{2}}{2}=K T$;

Para muitos propósitos é adequado utilizar um fluxo convencional $\Phi_{0}$ como:

$$
\Phi_{0}=n v_{0}
$$

Sendo,

$$
\begin{array}{ll}
n & \text { a densidade de nêutrons, } \\
v_{0} & \text { a velocidade na energia mais provável }(2200 \mathrm{~m} / \mathrm{s}) .
\end{array}
$$

Esta velocidade é escolhida por ser a velocidade na energia mais provável de uma distribuição de densidade maxwelliana à uma temperatura de $20,44^{\circ} \mathrm{C}$, e corresponde à uma energia de $0,025 \mathrm{eV}$.

\subsection{Espectro de Nêutrons Rápidos}

A distribuição de energia dos nêutrons produzidos no processo de fissão (rápidos) é mais conhecida como espectro de fissão. No núcleo de um reator o espectro de nêutrons é de forma geral pouco perturbado em relação ao espectro de fissão, mas essa perturbação acentua-se na sua vizinhança, à medida em que a distância entre o ponto considerado e o núcleo do reator aumenta. Existem várias formas de representar o espectro de nêutrons rápidos, sendo que uma das formas mais utilizadas é a de Watt, dada por: ${ }^{141 /}$

$$
S(E)=A e^{-E} \operatorname{senh} \sqrt{2 E}
$$


Sendo,

E a energia em $\mathrm{MeV}$,

$S(E) \quad$ o número de nêutrons por unidade de energia,

A constante de normalização $\sqrt{2 / \pi \cdot e}=0,484$.

Uma forma alternativa de expressar o espectro de fissão, a qual é também frequentemente utilizada é a forma Maxwelliana:

$$
S(E)=a \sqrt{E} e^{\left(\frac{-E}{\varepsilon}\right)}
$$

Onde $\varepsilon$ é a energia característica do processo e $a=2 / \sqrt{\left(\pi \varepsilon^{3}\right)}$.

As figuras 2.4 e 2.5 mostram o espectro de fissão $S(E)$ na forma Maxwelliana:

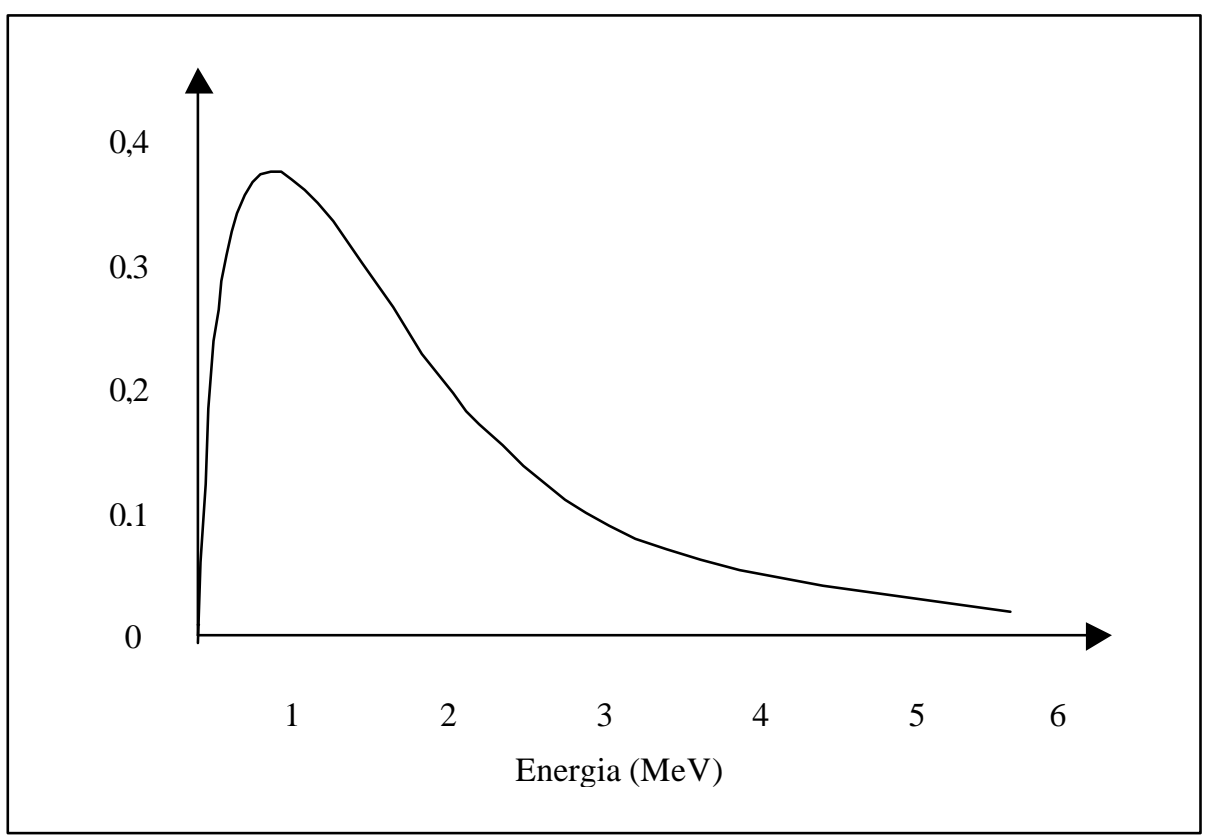

Figura 2.4 - Espectro de fissão $S(E)$ na forma Maxwelliana 


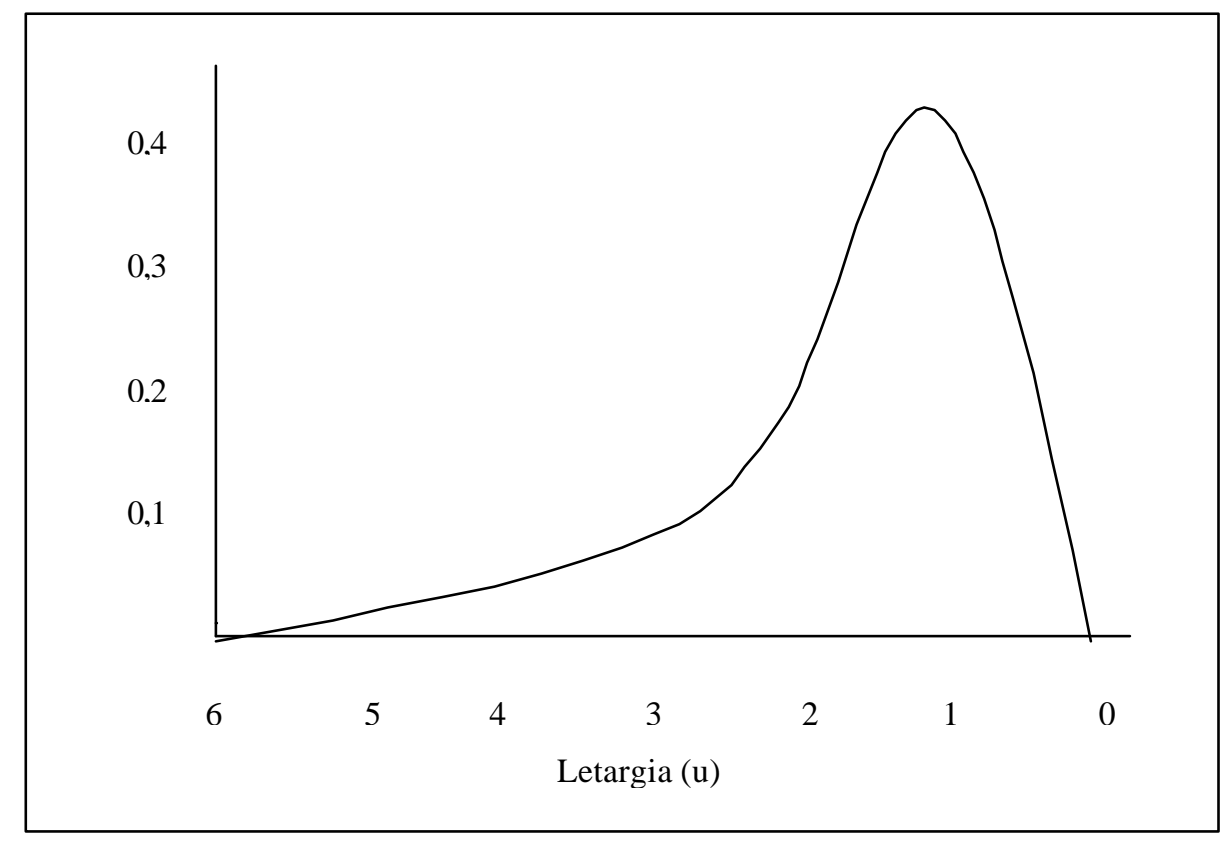

Figura 2.5 - Espectro de fissão $S(E)$ em unidades de Letargia

De forma geral o espectro de energia dos nêutrons de um reator nuclear não é representado pelo espectro de fissão devido ao processo de moderação e fuga de nêutrons, embora ele possa ser uma boa aproximação em regiões próximas a uma fonte de nêutrons para energias acima de $1,5 \mathrm{MeV}$.

Embora $S(E)$ seja o número de nêutrons emitidos por unidade de energia por unidade de tempo, o fluxo de nêutron não é obtido multiplicando-se $S(E)$ pela velocidade do nêutron, conforme pode ser visto a seguir: Considere um cubo $\delta A \delta r$, com $\delta A$ perpendicular a $r$, então o número de nêutrons entrando no cubo em unidade de tempo pode ser expresso matematicamente por:

$$
\omega(E)=\left(\frac{\delta A}{4 \pi \cdot r^{2}}\right) S(E)
$$

O intervalo de tempo que cada nêutron permanece no cubo, assumindo que não haja absorção, é dado por:

$$
\tau(E)=\frac{\delta r}{v}
$$


Onde $v$ é a velocidade correspondente ao nêutron de energia $E$. Portanto a densidade de nêutrons $n(E)$, no volume $\delta A \delta r$ é expressa por:

$$
n(E)=\frac{\omega(E) \tau(E)}{\delta A \delta r}=\frac{1}{4 \pi r^{2}} \frac{S(E)}{v}
$$

Portanto, o espectro de nêutrons rápidos é dado pela seguinte equação:

$$
\Phi(E)=n(E) v=\frac{S(E)}{4 \pi r^{2}}
$$

\subsection{Espectro de Nêutrons Intermediários}

Considerando um meio moderador infinito, homogêneo e não absorvedor, o espectro de nêutrons intermediários pode ser escrito da seguinte forma:

$$
\Phi(E)=\frac{Q}{\xi \eta \sigma_{s}} \frac{1}{E}
$$

Sendo,

$Q \quad$ a densidade de moderação,

$\eta \quad$ a densidade atômica do material,

$\sigma_{s}$ a seção de choque de espalhamento microscópica,

$\xi N \sigma_{s}$ é conhecido como poder de moderação,

$\frac{Q}{\xi N \sigma_{s}} \quad$ é o fluxo por intervalo logaritmo de energia, onde $\xi=\Delta n E$.

Desta forma conclui-se que o espectro de nêutrons intermediários varia na razão inversa de sua energia, ou seja, possui um comportamento $1 / E$. 
Dada a extensão de várias ordens de grandeza do espectro de nêutrons num reator, é normal substituir a energia por uma grandeza adimensional, chamada de letargia. A letargia $u$ é definida pela seguinte equação ${ }^{15 /}$ :

$$
d u=-d(\ln E)=-\frac{d E}{E}
$$

Ou seja:

$$
u=\ln \left(\frac{E_{0}}{E}\right)
$$

Sendo $\Phi(u)$ a densidade de fluxo de nêutrons por intervalo unitário de letargia, para um intervalo infinitesimal $d u$, tem-se que:

$$
\Phi(u) d u=-\Phi(E) d E
$$

O sinal negativo é introduzido na equação acima pelo fato da energia e a letargia serem grandezas anti-correlacionadas. A partir das equações (2.31) e (2.33), conclui-se que:

$$
\Phi(u)=E \Phi(E)
$$

\subsection{Medidas de Fluxo Térmico e Epitérmico}

A atividade de saturação induzida por nêutrons epitérmicos em um detector de nêutrons térmicos pode ser consideravelmente compartilhada do total da atividade de saturação do detector, ou seja, parte da atividade de saturação de uma folha nua irradiada é devida a nêutrons térmicos e parte a nêutrons epitérmicos. É possível descriminar estas duas faixas de energia utilizando-se um filtro neutrônico como uma cobertura de cádmio, por exemplo. 
O cádmio é um material que possui elevada seção de choque de absorção para nêutrons térmicos e praticamente desprezível para nêutrons epitérmicos. Portanto, coberturas de cádmio com espessura a partir de $\sim 0,75 \mathrm{~mm}$ atuam como filtros que absorvem quase todos os nêutrons com energia inferior à $0,5 \mathrm{eV}$, durante a irradiação, enquanto que a grande maioria dos nêutrons acima desta energia não são absorvidos pelo cádmio.

Assim, as taxas de reação podem ser divididas em dois termos, onde o primeiro representa a contribuição dos nêutrons térmicos e o segundo dos nêutrons epitérmicos. Para tal utiliza-se o conceito de Razão de Cádmio.

A razão de cádmio é definida como a razão entre as atividades de saturação da folha irradiada nua e da folha irradiada com cádmio, e é dada por:

$$
R_{C d}=\frac{A_{N u a}^{\infty}}{A_{C d}^{\infty}}
$$

$\mathrm{ou}$

$$
R_{C d}=\frac{A_{T}^{\infty}}{A_{C d}^{\infty}}=\frac{A_{t e r}^{\infty}+A_{C d}^{\infty}}{A_{C d}^{\infty}}=1+\frac{A_{t e r}^{\infty}}{A_{C d}^{\infty}}
$$

Sendo,

$A_{T}^{\infty} \quad$ Atividade de saturação total da folha nua irradiada;

$A_{t e r}^{\infty} \quad$ Atividade de saturação induzida por nêutrons térmicos;

$A_{C d}^{\infty} \quad$ Atividade de saturação da folha irradiada com cobertura de cádmio.

Considerando que o cádmio não é um filtro ideal, ou seja, ele não é totalmente transparente a nêutrons epitérmicos, absorvendo uma pequena fração destes, deve-se fazer a seguinte consideração: 


$$
A_{C d}^{\infty}=\frac{A_{e p i}^{\infty}}{F_{C d}}
$$

Onde $F_{C d}$ é o fator de cádmio, parâmetro dependente da espessura do cádmio utilizado como cobertura e das características da folha ativada.

Substituindo a expressão (2.36) em (2.35), têm-se:

$$
A_{e p i}^{\infty}=A_{\text {nua }}^{\infty} \frac{F_{C d}}{R_{C d}}
$$

e

$$
A_{\text {ter }}^{\infty}=A_{\text {nиа }}^{\infty}\left(1-\frac{F_{C d}}{R_{C d}}\right)
$$

Substituindo a equação (2.37) e (2.38) na equação (2.9) obtém-se o fluxo de nêutrons térmicos e epitérmicos:

$$
\Phi_{t e r}=\frac{A_{\text {nua }}^{\infty}\left(1-F_{C d} / R_{C d}\right)}{N_{A} \cdot \bar{\sigma}_{a c t} K_{t e r}}
$$

$\mathrm{e}$

$$
\Phi_{e p i}=\frac{A_{n u a}^{\infty} \cdot F_{C d}}{N_{A} I_{R} K_{e p i}}
$$

Sendo,

$K_{\text {ter }}$ fator de perturbação do fluxo térmico;

$K_{e p i}$ fator de perturbação do fluxo epitérmico;

O termo $I_{R}$ representa a integral de ressonância , que é definida por: 


$$
I_{R}=\int_{E_{C d}}^{\infty} \sigma_{a c t}(E) \frac{d E}{E}
$$

A integral de ressonância fornece o comportamento da seção de choque de ativação acima da energia de corte do cádmio $\left(E_{C d}\right)$.

O termo $\overline{\boldsymbol{\sigma}}_{a c t}$ é a seção de choque média de ativação, e é obtida através da expressão:

$$
\bar{\sigma}_{a c t}=\frac{\sqrt{\pi}}{2} \sigma_{a c t}\left(E_{0}\right) \sqrt{\frac{T_{0}}{T_{n}}} \cdot g\left(T_{n}\right)
$$

Sendo,

$$
\begin{array}{ll}
\sigma_{a c t}\left(E_{0}\right) & \text { seção de choque de ativação térmica à energia mais provável } \\
& (0,0253 \mathrm{eV}) ; \\
T_{0} & \text { temperatura correspondente à energia mais provável }(293,6 \mathrm{~K}) ; \\
T_{n} & \text { temperatura efetiva dos nêutrons, que nos fornece o desvio da } \\
& \text { distribuição térmica em relação à Maxwelliana; } \\
g\left(T_{n}\right) & \text { fator de Westcott }{ }^{/ 42 /} \text {, que leva em conta o desvio da seção de } \\
& \text { choque da forma } 1 / v .
\end{array}
$$

\subsection{Medidas de Fluxo Rápido}

Certas reações nucleares, como (n,p), (n,a), (n,n'), ocorrem somente se a energia do nêutron for acima de um limiar. Em muitos casos, estas reações lidam com produtos radioativos e podem ser utilizadas para determinar o espectro de energia dos nêutrons na região rápida de um reator. Como a densidade de fluxo de nêutrons rápidos e as seções de choque dos detectores de limiar são na maioria dos casos funções complexas da energia do nêutron, para obter a resposta dos detectores, a forma do espectro de nêutrons deve ser bem conhecida. Para tal, deve-se considerar algumas simplificações. 
A seção de choque de um detector de limiar deve ser representada por uma função que é igual a 0 abaixo do limiar de energia $E_{t}$ e é igual a $\sigma_{0}$ acima de energia $E_{t}$. Sendo assim, a taxa de reação medida pode ser escrita da seguinte forma:

$$
R=\sigma_{0} \int_{E_{t}}^{E} \Phi(E) d E
$$

Mas esta função só é uma boa aproximação para seções de choque reais. Portanto, na prática, outras aproximações são consideradas:

1 - Pode-se definir uma seção de choque média para o espectro de nêutrons de interesse, dada por:

$$
\bar{\sigma}=\frac{\int_{0}^{\infty} \sigma(E) \Phi(E) d E}{\int_{0}^{\infty} \Phi(E) d E}=\frac{R}{\int_{0}^{\infty} \Phi(E) d E}
$$

Da equação (2.44), o fluxo de nêutrons rápidos pode ser calculado:

$$
\int_{0}^{\infty} \Phi(E) d E=\frac{R}{\bar{\sigma}}
$$

2 - Pode-se definir uma seção de choque efetiva $\left(\sigma_{e f f}\right)$ e correspondente limiar de energia efetivo $E_{\text {eff }}$, de tal maneira que a taxa de reação real é dada por:

$$
\int_{0}^{\infty} \Phi(E) \sigma(E) d E=\sigma_{e f f} \cdot \int_{E_{e f f}}^{\infty} \Phi(E) d E
$$

Tanto a seção de choque efetiva, como o limiar de energia efetivo dependem da forma do espectro de nêutrons do reator. Neste caso o fluxo de nêutrons rápidos acima do limiar de energia efetivo é expresso da seguinte forma: 


$$
\Phi_{\text {eff }}\left(E_{\text {eff }}, \infty\right)=\frac{\int_{0}^{\infty} \Phi(E) \sigma(E) d E}{\sigma_{\text {eff }}}=\frac{R}{\sigma_{\text {eff }}}
$$

\subsection{Fatores de Correção para Perturbação do Fluxo de Nêutrons}

As folhas de ativação, devido às suas pequenas dimensões, produzem perturbações no campo neutrônico bem menores que os detectores convencionais. No entanto, quando se deseja obter o valor absoluto do fluxo neutrônico é necessário conhecer os fatores de correção de fluxo, a fim de se determinar o valor do mesmo sem os efeitos de perturbação.

Os fatores de perturbação de fluxo podem ser divididos em dois: Auto-blindagem $(G)$ e depressão de fluxo $(H)$.

O efeito de auto-blindagem se deve à atenuação do fluxo neutrônico das camadas externas da folha para as internas, provocando uma distribuição de fluxo não homogênea no detetor, tendo como consequiência uma diminuição na ativação da folha causada pela diminuição do fluxo neutrônico ${ }^{3 /}$.

O fator de auto-blindagem é definido como sendo a razão entre o fluxo médio no detector e o fluxo na superfície, e é matematicamente expresso por:

$$
G=\bar{\Phi} / \Phi_{S}
$$

O fator de auto-blindagem térmico pode ser calculado, assumindo um fluxo de nêutrons isotrópicos através da seguinte equação ${ }^{133,36 /}$ :

$$
G_{t e r}=\frac{1}{2 x}\left(1-2 E_{3}(x)\right)
$$

Onde $x=\Sigma_{a} e$, sendo $\Sigma_{a}$ a seção de choque macroscópica média de absorção , $e$ é a espessura da folha e $E_{3}$ é a função exponencial de terceira ordem, que pode ser dada por $/ 19 /$ : 


$$
E_{3}(x)=\frac{1}{2}\left[e^{-x}(1-x)+x^{2} E_{1}(x)\right]
$$

Onde $E_{l}$ é a função exponencial de primeira ordem e pode ser expandida em série:

$$
E_{1}(x)=x-\frac{x^{2}}{2.2 !}+\frac{x^{3}}{3.3 !}+\ldots \ldots . . \ln (x)-\gamma
$$

Onde $\gamma$ é a constante de Euler.

$\mathrm{Na}$ região epitérmica a seção de choque de ativação pode ser dividida na componente $1 / v$ e, em uma ou mais ressonâncias descritas pela fórmula de Breit-Wigner. A auto-blindagem da parte $1 / v$ é desprezível quando comparada com a auto-blindagem ressonante, tendo em vista os altos valores da seção de choque na ressonância.

O fator de auto-blindagem epitérmico num campo isotrópico é dado por:

$$
G_{e p i}(\tau)=\frac{\tau}{2} \int_{\tau / 2}^{\infty} z^{-2} e^{-z}\left(I_{0}(z)+I_{1}(z)\right) d z
$$

onde $\tau=\Sigma_{a} e, I_{0} e I_{l}$ são as funções de Bessel modificadas de grau zero e grau um, de primeira ordem.

Uma função de aproximação simples para o fator de auto-blindagem epitérmico, válida para quaisquer espessuras é dada por Bensch ${ }^{/ 1 /}$ :

$$
G_{e p i}=\frac{1}{\sqrt{2 \Sigma_{R} d+1}}
$$

onde $\Sigma_{R}=N \sigma_{R}$ sendo $\sigma_{R}$ o valor máximo da seção de choque de ressonância corrigido quanto ao alargamento Doppler da ressonância, $\mathrm{N}$ o número de átomos alvo/ $\mathrm{cm}^{3}$ e d a espessura do detector.

O efeito de depressão de fluxo se deve ao fato da folha de ativação ocupar um espaço anteriormente ocupado pelo meio moderador; com isso, além da folha não permitir 
no local a mesma taxa de espalhamento, ela absorve nêutrons, provocando ao seu redor uma depressão no fluxo neutrônico.

O fator de depressão de fluxo $H$ pode ser definido como:

$$
H=\Phi_{S} / \Phi_{M}
$$

Sendo,

$\Phi_{S} \quad$ o fluxo de nêutrons junto à superfície do moderador;

$\Phi_{M} \quad$ o fluxo não perturbado no moderador.

O fator de depressão de fluxo dado pela teoria de transporte pode ser escrito $\operatorname{como}^{29 /}$ :

$$
H(\gamma, \tau)=\frac{1}{1+\left(\frac{1}{2}-E_{3}(\tau)\right) g(R, \gamma)}
$$

onde $\gamma=\lambda / \lambda_{s}$ é a razão entre o livre caminho médio total e o livre caminho médio de espalhamento e $g(R, \gamma)$ é o fator de Skyrme modificado por Kitch e Eldridge ${ }^{136 /}$ para uma folha na forma de um disco de raio $r$ tal que:

$$
g(r, \gamma)=\frac{3}{2}\left(\frac{L}{\gamma}\right) S\left(\frac{2 r}{L}\right)-K\left[\left(\frac{2 r}{\lambda}\right) \gamma\right] r_{g}
$$

sendo $L$ o comprimento de difusão, $r_{g}$ o fator de correção de Skyrme-Ritch-Eldridge, $S$ a função de Skyrme, é dada por ${ }^{136 /}$ :

$$
S(x)=1-\frac{4}{x} \int_{0}^{1} e^{-x t}\left(1-t^{2}\right) d t
$$

onde $x=2 r / L$ 
Assim, o fator de perturbação de fluxo térmico $K_{t}$ é dado por,

$$
K_{t e r}=G_{t e r} H
$$

e o fator de perturbação epitérmico $K_{e p i}$ é dado basicamente pelo fator de auto blindagem ressonante $G_{\text {epi }}$.

Neste trabalho os fatores de auto-blindagem foram calculados pelo método de monte carlo a partir da modelagem das folhas de ativação no núcleo do Reator IPEN/MB01, utilizando o programa computacional MCNP-4C ${ }^{8 /}$, conforme podemos visualizar no Anexo F. 


\section{MATERIAIS E MÉTODOS EXPERIMENTAIS}

O Reator IPEN/MB-01 é um reator de potência zero especialmente projetado para a medida de uma grande variedade de parâmetros de Física de Reatores, objetivando correlacionar teoria ao experimento. Após quase 18 anos de operação e mais de 1900 operações (ano 2006), vários dos experimentos realizados no Reator IPEN/MB-01 se tornaram padrões de comparação internacional ("Benchmark").

O núcleo do reator em sua configuração padrão retangular consiste de um arranjo de 28x26 varetas combustíveis, enriquecida a 4,3\% com um encamisamento de aço inox (304), inseridas dentro de um tanque moderador de água leve. Uma completa descrição do reator pode ser obtida no Anexo A.

\subsection{Seleção das Folhas de Ativação}

As folhas de ativação a serem utilizadas para medida do espectro devem ser selecionadas de acordo com os seguintes critérios ${ }^{149 /}$ :

1. Folhas com alto grau de pureza, a fim de se evitar impurezas e consequentemente reações indesejadas,

2. Elementos quimicamente estáveis, na forma metálica,

3. Folhas em pequenas dimensões ou na forma de ligas infinitamente diluídas,

4. As folhas devem ter uma seção de choque de ativação suficiente para se medir uma taxa de contagens no sistema de detecção,

5. Produto formado na reação nuclear,

6. Meia-vida do produto formado,

7. Faixa de Atuação no espectro de energia dos nêutrons. 
Antes da irradiação, as folhas foram devidamente limpas com álcool isopropílico, a fim de se evitar impurezas que possam ocasionar interferências nas medidas. Em seguida foram pesadas em balança de alta precisão. Suas espessuras e diâmetros, são dados normalmente fornecidos pelos fabricantes.

\subsection{Característica das Folhas de Ativação}

As tabelas 3.1, 3.2, 3.3 e 3.4 mostram as características dos 16 detectores de ativação selecionados e utilizados para a obtenção do espectro:

Tabela 3.1. Reações nucleares e faixa de resposta dos detectores de ativação térmicos.

\begin{tabular}{|c|c|c|}
\hline Folhas & Reação Nuclear & $\begin{array}{c}\text { Faixa de Atuação no } \\
\text { Espectro }(\text { MeV })\end{array}$ \\
\hline $\mathrm{Sc}$ & $\mathrm{Sc}^{45}(\mathrm{n}, \gamma) \mathrm{Sc}^{46}$ & $1,0 \times 10^{-9}$ \\
\hline $\mathrm{Au}$ & $\mathrm{Au}^{197}(\mathrm{n}, \gamma) \mathrm{Au}^{198}$ & $1,0 \times 10^{-6}$ \\
\hline
\end{tabular}

Tabela 3.2. Reações nucleares e faixa de resposta dos detectores de ativação intermediários.

\begin{tabular}{|c|c|c|}
\hline Folhas & Reação Nuclear & $\begin{array}{c}\text { Faixa de Atuação no } \\
\text { Espectro }(\mathrm{MeV})\end{array}$ \\
\hline $\mathrm{U}$ & $\mathrm{U}^{238}(\mathrm{n}, \tilde{\mathrm{a}}) \mathrm{U}^{239} \quad \mathrm{~Np}^{239}$ & $1,2 \times 10^{-7} \_2,2 \times 10^{-4}$ \\
\hline Sc & $\mathrm{Sc}^{45}(\mathrm{n}, \gamma) \mathrm{Sc}^{46 *}$ & $2,5 \times 10^{-7} \_4,5 \times 10^{-3}$ \\
\hline $\mathrm{Au}$ & $A u^{197}(n, \gamma) A u^{198 *}$ & $2,8 \times 10^{-6}{ }^{-6} 3,0 \times 10^{-5}$ \\
\hline $\mathrm{U}$ & $\mathrm{U}^{238}(\mathrm{n}, \tilde{\mathrm{a}}) \mathrm{U}^{239} \quad \mathrm{~Np}^{239} *$ & $6,3 \times 10^{-6}{ }^{-6} 2,2 \times 10^{-4}$ \\
\hline
\end{tabular}

*Folhas irradiadas com cobertura de cádmio; 
Tabela 3.3. Reações nucleares e faixa de resposta dos detectores de ativação rápidos.

\begin{tabular}{|c|c|c|}
\hline Folhas & Reação Nuclear & $\begin{array}{c}\text { Faixa de Atuação no } \\
\text { Espectro }(\mathrm{MeV})\end{array}$ \\
\hline In & $\operatorname{In}^{115}\left(\mathrm{n}, \mathrm{n}^{\prime}\right) \operatorname{In}^{115 \mathrm{~m}}$ & $1,0 \times 10^{0} \_6,7 \times 10^{0}$ \\
\hline In & $\operatorname{In}^{115}\left(\mathrm{n}, \mathrm{n}^{\prime}\right) \operatorname{In}^{115 \mathrm{~m}_{*}}$ & $1,0 \times 10^{0}$ \\
\hline $\mathrm{Ni}$ & $\mathrm{Ni}^{58}(\mathrm{n}, \mathrm{p}) \mathrm{Co}^{58}$ & $1,8 \times 10^{0}$ \\
\hline $\mathrm{Ni}$ & $\mathrm{Ni}^{58}(\mathrm{n}, \mathrm{p}) \mathrm{Co}^{58 *}$ & $1,8 \times 10^{0} \_8,2 \times 10^{0}$ \\
\hline $\mathrm{Ti}$ & $\mathrm{Ti}^{47}(\mathrm{n}, \mathrm{p}) \mathrm{Sc}^{47}$ & $1,8 \times 10^{0} \_8,2 \times 10^{0}$ \\
\hline $\mathrm{Ti}$ & $\mathrm{Ti}^{47}(\mathrm{n}, \mathrm{p}) \mathrm{Sc}^{47} *$ & $1,8 \times 10^{0}$ \\
\hline $\mathrm{Ti}$ & $\mathrm{Ti}^{48}(\mathrm{n}, \mathrm{p}) \mathrm{Sc}^{48 *}$ & $5,5 \times 10^{0}$ \\
\hline $\mathrm{Al}$ & $\mathrm{Al}^{27}(\mathrm{n}, \alpha) \mathrm{Na}^{24 *}$ & $6,7 \times 10^{0}$ \\
\hline $\mathrm{Mg}$ & $\mathrm{Mg}^{24}(\mathrm{n}, \mathrm{p}) \mathrm{Na}^{24}$ & $1,3 \times 10^{+1}$ \\
\hline $\mathrm{Mg}$ & $\mathrm{Mg}^{24}(\mathrm{n}, \mathrm{p}) \mathrm{Na}^{24 *}$ & $6,7 \times 10^{0}$ \\
\hline
\end{tabular}

*Folhas irradiadas com cobertura de cádmio;

Tabela 3.4. Massa e espessura das folhas irradiadas.

\begin{tabular}{|c|c|c|}
\hline Folhas Irradiadas & Massa $(\mathbf{g})$ & Espessura $(\mathbf{m m})$ \\
\hline $\mathrm{Au}^{197}$ & 0,02727 & 0,0254 \\
\hline $\mathrm{Au}^{197}$ c/ cádmio & 0,02744 & 0,0254 \\
\hline $\mathrm{U}^{238}$ & 0,12265 & 0,1016 \\
\hline $\mathrm{U}^{238}$ c/ cádmio & 0,11103 & 0,1016 \\
\hline $\mathrm{Sc}^{45}$ & 0,05400 & 0,127 \\
\hline $\mathrm{Sc}^{45} \mathrm{c} /$ cádmio & 0,05218 & 0,127 \\
\hline $\mathrm{Al}^{27} \mathrm{c} /$ cádmio & 0,04460 & 0,127 \\
\hline $\mathrm{Ti}^{48} \mathrm{c} /$ cádmio & 0,14170 & 0,254 \\
\hline $\mathrm{Ni}$ & 0,28500 & 0,254 \\
\hline $\mathrm{Ni}^{58} \mathrm{c} /$ cádmio & 0,28500 & 0,254 \\
\hline $\mathrm{Mg}$ & 0,03152 & 0,127 \\
\hline $\mathrm{Mg}^{24} \mathrm{c} /$ cádmio & 0,03152 & 0,127 \\
\hline $\mathrm{In}$ & 0,12200 & 0,127 \\
\hline $\mathrm{In}^{115} \mathrm{c} /$ cádmio & 0,1208 & 0,127 \\
\hline $\mathrm{Ti}{ }^{47}$ & 0,14463 & 0,254 \\
\hline $\mathrm{Ti}^{47} \mathrm{c} /$ cádmio & 0,14050 & 0,254 \\
\hline
\end{tabular}




\subsection{Condições das Irradiações}

Antes de inserir as folhas no reator, elas foram acopladas à uma régua de acrílico do dispositivo articulado que permite que as mesmas fiquem posicionada exatamente na posição central do núcleo do reator, na região moderadora, região onde têm-se o maior fluxo de nêutrons rápidos, no interior do núcleo. Este dispositivo assegura que todas as folhas sejam irradiadas na mesma posição.

O tempo de irradiação foi calculado levando-se em conta a meia-vida do produto formado na reação nuclear e a seção de choque de ativação do alvo irradiado. Em algumas folhas foram utilizadas "caixas" de cádmio como cobertura, a fim de evitar-se interferência de nêutrons térmicos. As seções de choque de ativação dos produtos formados nas reações nucleares, bem como a meia-vida dos produtos formados podem ser vistos na tabela (3.5):

Tabela 3.5 - Seção de choque de ativação do núcleo alvo, meia-vida dos produtos formados e tempo de irradiação dos detectores.

\begin{tabular}{|c|c|c|c|}
\hline $\begin{array}{c}\text { Reação Nuclear/Produto } \\
\text { Formado }\end{array}$ & $\begin{array}{c}\text { Tempo de } \\
\text { Irradiação } \\
\text { (horas) }\end{array}$ & $\begin{array}{c}\text { Seção de Choque de } \\
\text { Ativação do Núcleo } \\
\text { Alvo (barns) }\end{array}$ & $\begin{array}{c}\text { Meia-Vida dos } \\
\text { Produtos Formados } \\
\text { (horas) }\end{array}$ \\
\hline $\mathrm{Au}^{197}(\mathrm{n}, \gamma) \mathrm{Au}^{198}$ & $1 \mathrm{~h}$ & 99,57 & 64,56 \\
\hline $\mathrm{Au}^{197}(\mathrm{n}, \gamma) \mathrm{Au}^{198 *}$ & $1 \mathrm{~h}$ & 15630 & 64,56 \\
\hline $\mathrm{Sc}^{45}(\mathrm{n}, \gamma) \mathrm{Sc}^{46}$ & $1 \mathrm{~h}$ & 27,21 & 2011,92 \\
\hline $\mathrm{Sc}^{45}(\mathrm{n}, \gamma) \mathrm{Sc}^{46 *}$ & $1 \mathrm{~h}$ & 11,18 & 2011,92 \\
\hline $\mathrm{U}^{238}(\mathrm{n}, \tilde{\mathrm{a}}) \mathrm{U}^{239} \mathrm{~Np}^{239}$ & $1 \mathrm{~h}$ & 2,715 & 56,4 \\
\hline $\mathrm{U}^{238}(\mathrm{n}, \tilde{\mathrm{a}}) \mathrm{U}^{239} \mathrm{~Np}^{239} *$ & $1 \mathrm{~h}$ & 277,4 & 56,4 \\
\hline $\mathrm{Ti}^{48}(\mathrm{n}, \mathrm{p}) \mathrm{Sc}^{48 *}$ & $6 \mathrm{~h}$ & $2,59.10^{-4}$ & 44 \\
\hline $\mathrm{Ti}^{47}(\mathrm{n}, \mathrm{p}) \mathrm{Sc}^{47}$ & $8 \mathrm{~h}$ & $1,76.10^{-2}$ & 80,16 \\
\hline $\mathrm{Ti}^{47}(\mathrm{n}, \mathrm{p}) \mathrm{Sc}^{47 *}$ & $3 \mathrm{~h}$ & $1,76.10^{-2}$ & 1728 \\
\hline $\mathrm{Ni}^{58}(\mathrm{n}, \mathrm{p}) \mathrm{Co}^{58}$ & $2 \mathrm{~h}$ & $62,43.10^{-3}$ & 1728 \\
\hline $\mathrm{Ni}^{58}(\mathrm{n}, \mathrm{p}) \mathrm{Co}^{58 *}$ & $2 \mathrm{~h}$ & $62,43.10^{-3}$ & 15,06 \\
\hline $\mathrm{Mg}^{24}(\mathrm{n}, \mathrm{p}) \mathrm{Na}^{24}$ & $3 \mathrm{~h}$ & $1,47.10^{-3}$ & 15,06 \\
\hline $\mathrm{Mg}^{24}(\mathrm{n}, \mathrm{p}) \mathrm{Na}^{24 *}$ & $6 \mathrm{~h}$ & $1,47.10^{-3}$ & 4,5 \\
\hline $\mathrm{In}^{115}(\mathrm{n}, \mathrm{n}) \mathrm{In}^{115 \mathrm{~m}}$ & $1 \mathrm{~h}$ & $183.10^{-3}$ & 4,5 \\
\hline $\mathrm{In}^{115}\left(\mathrm{n}, \mathrm{n}\right.$ ') $\mathrm{In}^{115 \mathrm{~m} *}$ & $1 \mathrm{~h}$ & $183.10^{-3}$ & 15,06 \\
\hline $\mathrm{Al}^{27}(\mathrm{n}, \alpha) \mathrm{Na}^{24 *}$ & $1 \mathrm{~h}$ & $6,86.10^{-4}$ & \\
\hline
\end{tabular}

*Folhas irradiadas com cobertura de cádmio. 
Todas as irradiações foram realizadas à potência de 100 Watts, na posição central do núcleo do reator, no moderador e foram normalizadas entre sí, através do registro da contagem do canal 10 do reator, durante o tempo de irradiação, distante $40 \mathrm{~cm}$ do núcleo em sua face leste, trata-se de um detector tipo pulso de Boro-10. Com isso qualquer pequena flutuação na potência do reator de uma irradiação para a outra pode ser corrigida e normalizada.

As barras de controle $\mathrm{BC} 1$ e $\mathrm{BC} 2$ são inseridas à mesma altura no núcleo do reator, durante todas as irradiações, para manter as condições de simetria na distribuição do fluxo de nêutrons no núcleo do Reator IPEN/MB-01.

A figura 3.1 mostra a posição de irradiação das amostras, bem como a configuração do núcleo do reator utilizada em todas as irradiações: 


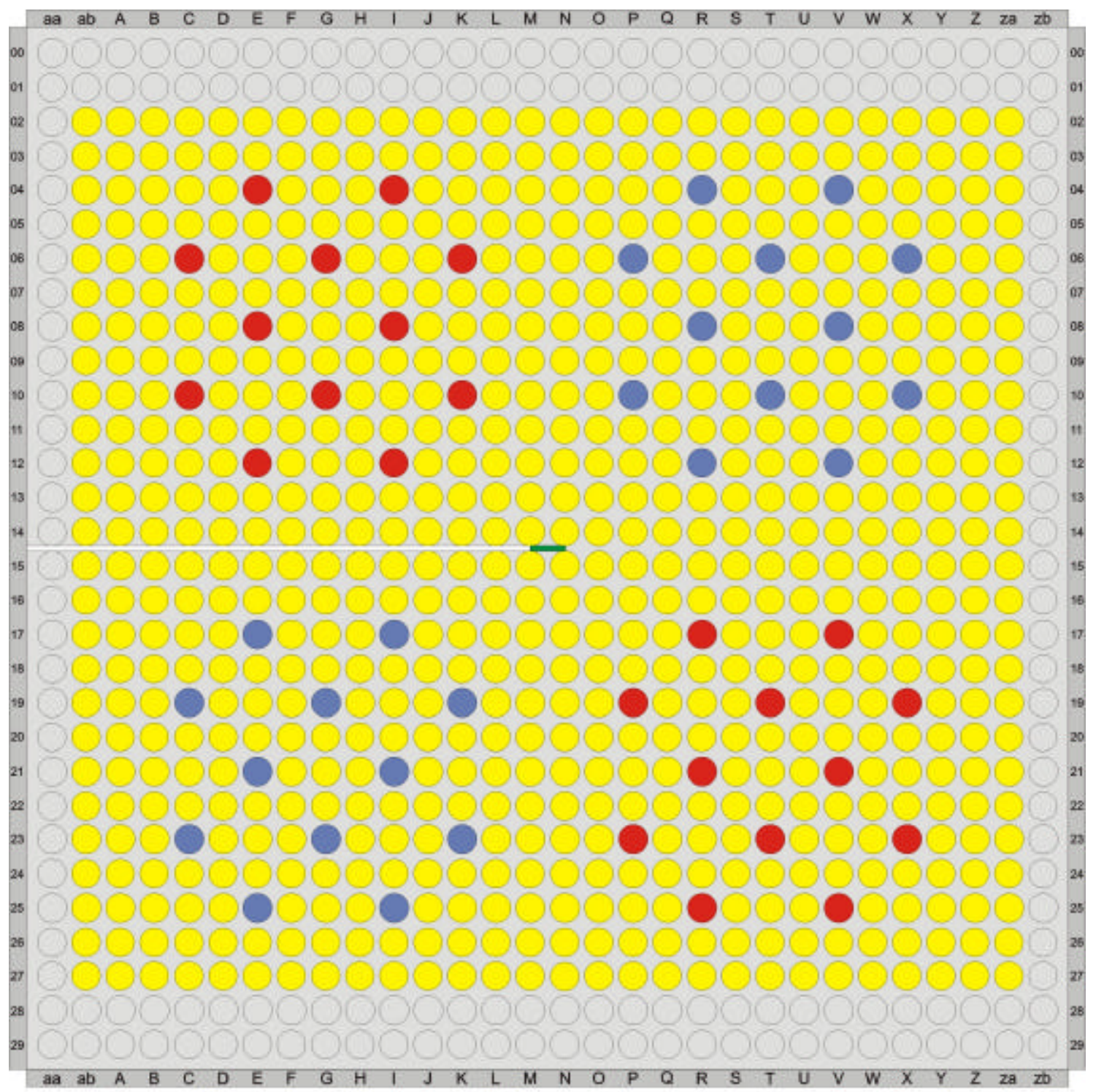

\section{LEGENDA:}

Vareta Combustivel

Vareta Absorvedora de Controle

- Vareta Absorvedora de Segurança

- Posiçăo de Irradiação das Folhas de Ativaçăo

- Règua de Lucite Acoplada ao Dispositivo Articulado

Figura 3.1 Representação da Configuração de Núcleo Retangular (26x28 Varetas) 


\subsection{Correções Experimentais}

Durante as irradiações dos detectores de ativação, devem-se realizar algumas correções experimentais, devido à rampa de subida de potência , ao fator de normalização monitorado pelo canal 10 da instrumentação nuclear, que monitora pequenas diferenças de potências entre irradiações distintas e à auto-absorção nas folhas.

\subsubsection{Fator de Normalização}

Devido às pequenas variações de potência existentes entre as diversas operações de irradiações dos detectores de ativação, é necessário normalizar a condição de irradiação ${ }^{14 /}$.

Este fator é calculado através de aquisições de contagens do canal 10 da instrumentação nuclear, a cada 5 minutos de irradiação em tempos de aquisição de 6 segundos.

É fundamental que durante a irradiação, as barras de controle do reator estejam igualmente inseridas no núcleo do reator, mantendo assim constante a distribuição espacial do fluxo de nêutrons ao longo do comprimento ativo do detector B-10 do canal 10, bem como na folha de ativação irradiada.

\subsubsection{Fator de Rampa}

A rampa de subida de potência do reator varia de irradiação para irradiação, sendo assim é necessário descontar a contribuição na taxa de reação induzida nas folhas de ativação.

Matematicamente o fator de rampa é expresso por ${ }^{/ 4 /}$ : 


$$
F_{R p}=\frac{\frac{1}{\lambda \cdot\left(e^{T r} / T\right) \cdot\left(1-e^{-\lambda t}\right)}}{\lambda\left(e^{T r} / T\right) \cdot\left(1-e^{-\lambda t}\right)+W}
$$

Onde,

$$
W=\frac{T}{(1+\lambda T) \lambda \cdot\left(e^{T r} / T\right) \cdot\left(e^{-\lambda t}\right)-\left(e^{-\lambda T r}\right)}
$$

Sendo,

T Período de subida de potência,

$\lambda$ Constante de decaimento do radionuclídeo formado,

$\operatorname{Tr}$ tempo de subida da rampa de potência,

$t$ tempo de irradiação do detector de ativação.

Por simplicidade pode-se utilizar a equação (3.3), que deriva das equações (3.1) e (3.2) e apresenta resultados muito próximos ao das equações mencionadas:

$$
F r=\frac{1}{1+\frac{T}{t}}
$$

O tempo de subida da rampa de potência foi obtido através de um cronômetro, após a mudança dos canais de controle do reator de partida para potência. Assim, o cronômetro é acionado em 100 miliwatts e travado na potência de irradiação de 100 watts. O período correspondente a rampa de subida de potência é determinado através da seguinte equação:

$$
T=\frac{\operatorname{Tr}}{\operatorname{Ln}\left(\frac{P}{P_{0}}\right)}
$$


Sendo,

$P \quad$ potência final de irradiação $(100 \mathrm{~W})$,

$P_{0} \quad$ potência inicial em que se dá inicio a cronometragem $(100 \mathrm{~mW})$

$\operatorname{Tr} \quad$ Tempo de subida da rampa de potência cronometrado entre $P_{0}$ e $P$.

\subsubsection{Correção para Auto-Absorção}

Outra importante correção, que se aplica aos detectores de ativação irradiados, é a correção quanto à auto-absorção gama. Esta correção é realizada através da seguinte expressão:

$$
f_{a b s}=\frac{1}{T}
$$

Onde,

$$
T=\frac{S\left[1-e^{-(\mu / \rho) \cdot(m / s)}\right]}{\left(\frac{\mu}{\rho}\right) m}
$$

Sendo,

$f_{a b s} \quad$ o fator de auto-absorção gama;

$\mu \quad$ o coeficiente de atenuação linear;

$S \quad$ a área superficial da folha;

$\rho \quad$ a densidade do material da folha;

$m \quad$ a massa da folha;

$\mu / \rho$ Coeficiente de atenuação de massa. 


\subsection{Espectrometria Gama}

Após a irradiação, as folhas de ativação são levadas ao laboratório, para se realizar a espectrometria gama das mesmas, e determinar a taxa de contagem ao término da irradiação, parâmetro diretamente proporcional a atividade da folha neste mesmo período de tempo. Para tal, utiliza-se um sistema de contagem composto de um detector semicondutor de germânio hiper puro com eficiência intrínseca de $40 \%$ e eletrônica associada (amplificador, fonte de alta tensão, multicanal e microcomputador). O sistema é calibrado em energia usando fontes radioativas padrões de Eu-152, Ba-133, Co-60 , Cs137, e sua estabilidade e qualidade são testados rotineiramente com fontes padrões, como também são realizados testes estatísticos como por exemplo o qui-quadrado.

3.5.1. Cálculo da Eficiência do Sistema de Detecção HPGe para as folhas irradiadas

Foi obtida a curva de Eficiência a partir da fonte radioativa padrão de Eu-152, para a $5^{\mathrm{a}}, 6^{\mathrm{a}}$ e $7^{\mathrm{a}}$ gaveta do sistema de detecção HPGE, respectivamente distantes do detector 6,9 $\mathrm{cm}, 5,9 \mathrm{~cm}$ e 4,9 cm. Tal fonte possuía uma atividade de $13,3 \mathrm{kBq}$ em 01 de março de 1991. A eficiência de um sistema de detecção pode ser expressa matematicamente por:

$$
\varepsilon=\frac{\lambda C e^{\lambda t_{e}}}{A I\left(1-e^{-\lambda t_{c}}\right)}
$$

Onde,

$\varepsilon \quad$ é a eficiência do sistema para o material irradiado,

$\lambda \quad$ é a constante de decaimento,

C é o número de contagens líquidas,

$t_{e} \quad$ é o tempo de espera para contagem (intervalo de tempo entre o término da irradiação e o início das contagens no detector),

A é a atividade da fonte,

I é a abundância gama do fotopico de energia,

$t_{c} \quad$ é o tempo de contagem no detector. 
Considerando que o $t_{c}$ e o $t_{e}$ são parâmetros definidos na estratégia do experimento e que $\lambda, A$ e $I$ são obtidos por meio de bibliotecas de dados nucleares, torna-se simples o cálculo da eficiência. A fim de simplificar o cálculo das incertezas dos valores da eficiência pode-se fazer uma aproximação expandindo o termo $e^{-\lambda t_{c}}$ em série de Taylor, até a $1^{\mathrm{a}}$ ordem, conforme descrito abaixo:

$$
e^{-\lambda t_{c}}=1-\lambda t \quad \therefore \quad\left(1-e^{-\lambda t_{c}}\right)=\lambda t_{c} \quad \rightarrow \quad t_{c}=L T(\text { LiveTime }) \quad \therefore \quad 1-e^{-\lambda t_{c}}=\text { LT. } \lambda
$$

Esta aproximação pode ser feita considerando que na maioria dos casos o tempo de contagem é muito menor do que o inverso da constante de decaimento, ou seja, considerase que a atividade do detector é constante durante o tempo de contagem.

Após as devidas substituições a equação da eficiência pode ser reescrita por:

$$
\varepsilon=\frac{C e^{\lambda t_{e}}}{A \cdot I \cdot L T}
$$

O cálculo das incertezas pode ser realizado então, usando o método matemático de propagação de erros, conforme a equação abaixo:

$$
\left(\frac{\boldsymbol{\delta}_{\varepsilon}}{\varepsilon}\right)^{2}=\left(\frac{\boldsymbol{\delta}_{c}}{C}\right)^{2}+\left(\frac{\boldsymbol{\delta}_{A}}{A}\right)^{2}+\left(\frac{\boldsymbol{\delta}_{I}}{I}\right)^{2}+\left(\boldsymbol{\delta}_{\lambda} t_{e}\right)^{2}
$$

Onde $\delta$ representa o desvio-padrão de cada grandeza apresentada na equação (3.7).

As figuras 3.2, 3.3 e 3.4 mostram a curva de eficiência para a $5^{\mathrm{a}}, 6^{\mathrm{a}}$ e $7^{\mathrm{a}}$ gavetas do sistema de detecção, obtidas através da fonte radioativa de Eu-152: 
Figura 3.2 - Curva de Eficiência da $5^{\text {a }}$ gaveta com fonte de Eu-152

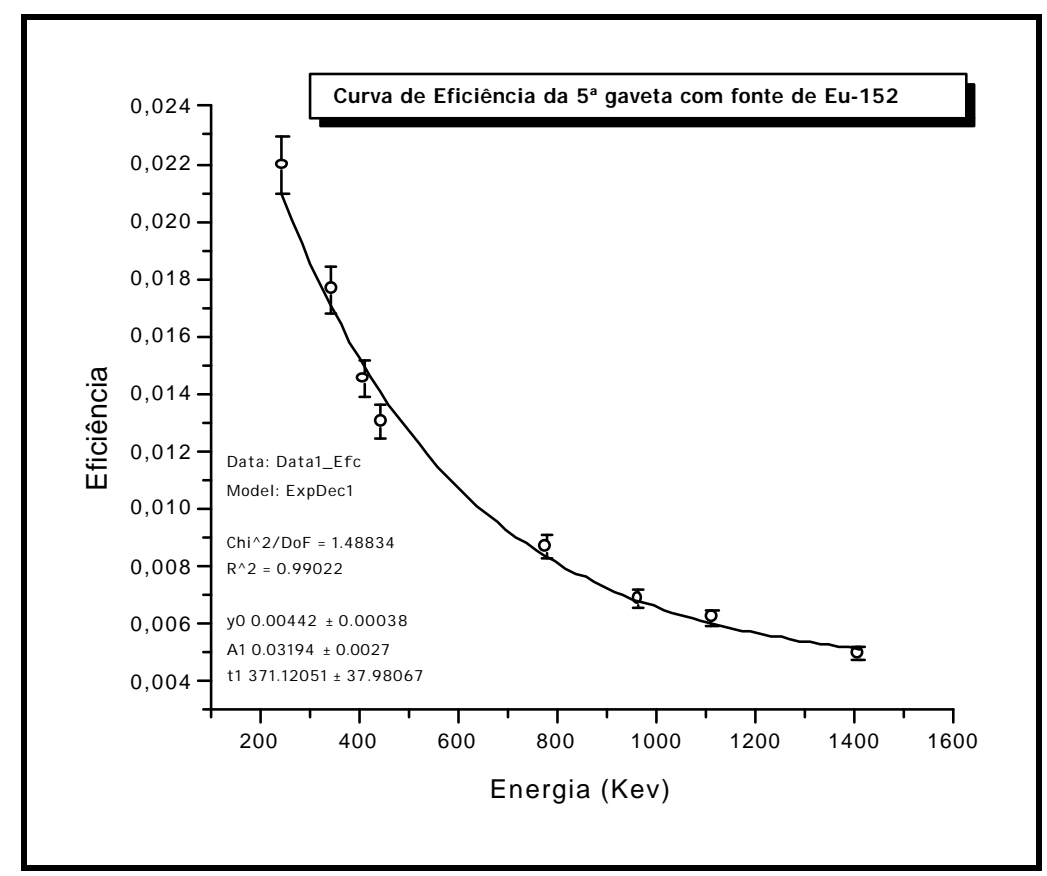

Figura 3.3 - Curva de Eficiência da $6^{\text {a }}$ gaveta com fonte de Eu-152

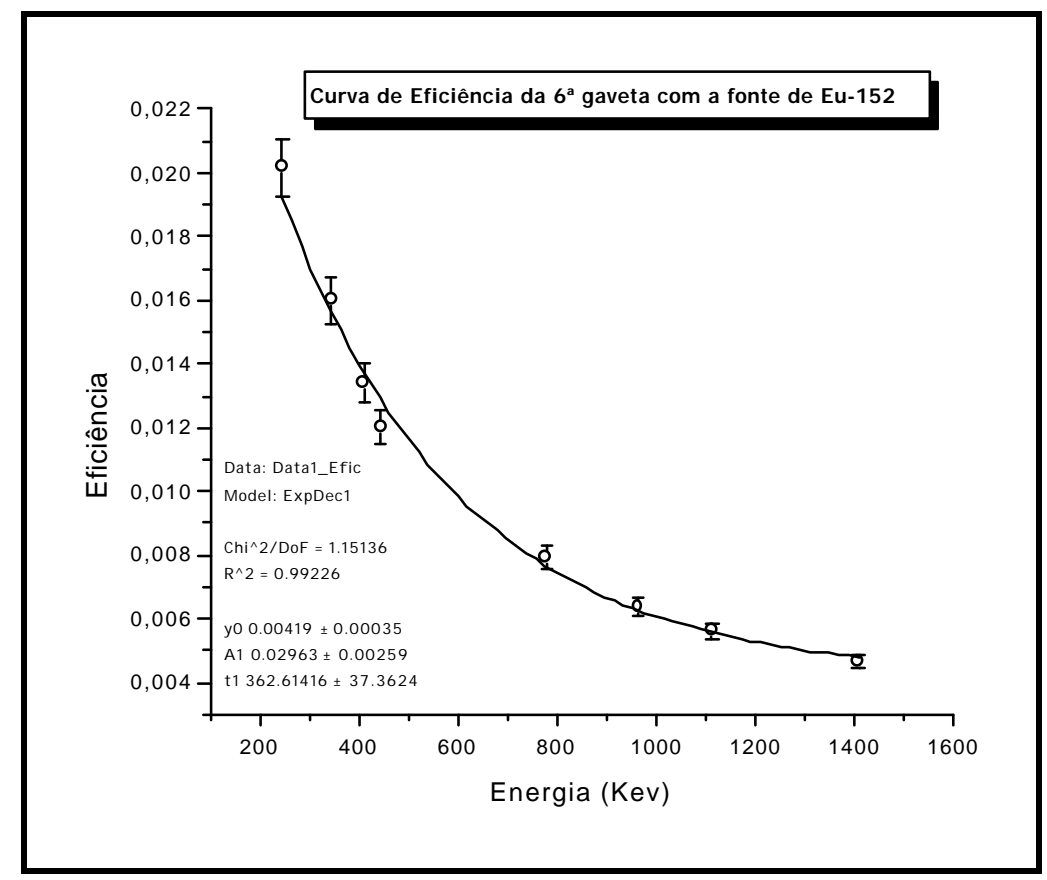


Figura 3.4 - Curva de Eficiência da $7^{\text {a }}$ gaveta com fonte de Eu-152

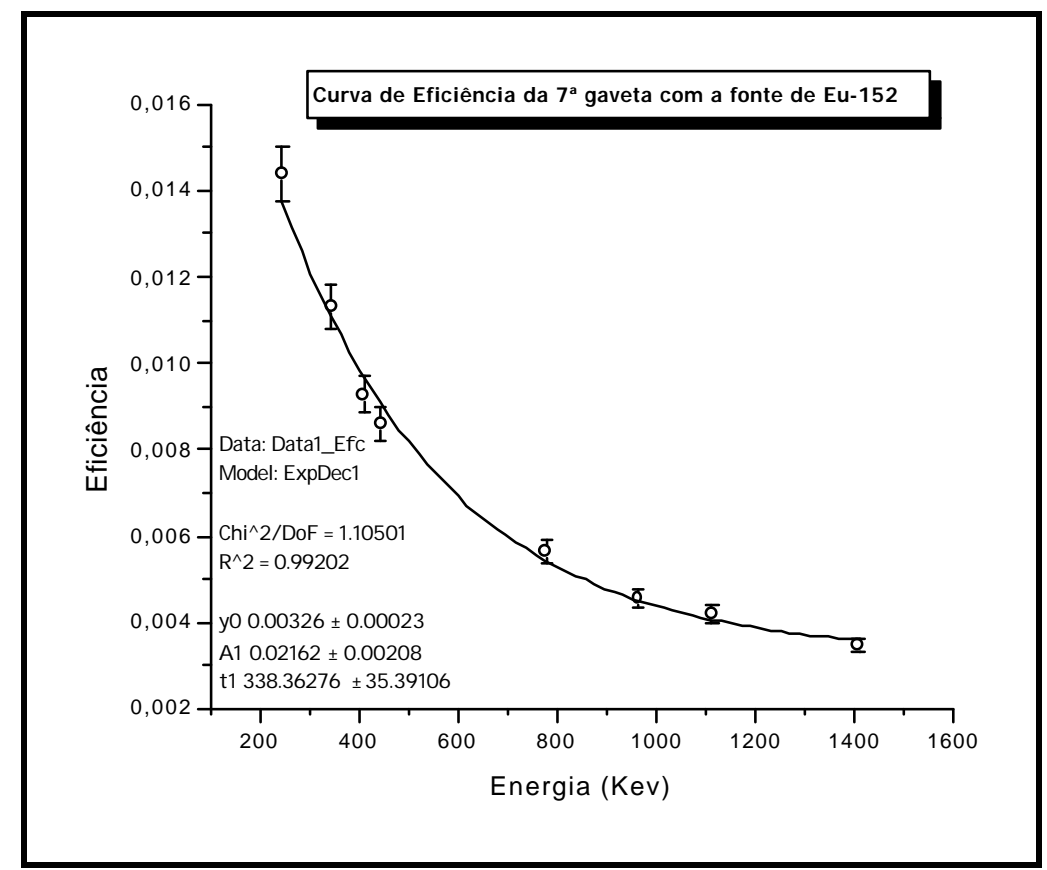

\subsubsection{Espectrometria Gama das Folhas Irradiadas}

Após a irradiação e retirada do dispositivo articulado do núcleo do reator, a folha é removida do dispositivo e levada para contagem no sistema de detecção HPGe, pelo qual é obtida a taxa de contagens líquidas, parâmetro diretamente proporcional à atividade da folha irradiada no mesmo período de tempo. Após determinada a atividade das folhas, é possível determinar a atividade de saturação por núcleo alvo, um dos principais parâmetros que deve ser informado na entrada do código SANDBP.

\subsubsection{Determinação dos parâmetros de entrada do código SANDBP ${ }^{138 /}$}

$\mathrm{Na}$ entrada do código deve ser especificado uma série de parâmetros como: o tipo de iteração realizada, a quantidade de folhas irradiadas, a descrição das folhas, o número de grupos de energia do espectro, o número limite de iterações, os critérios de descarte (quando deve parar o processo iterativo), as extrapolações para as regiões térmica e rápida (quando utilizadas), o tratamento das incertezas (método de Monte Carlo) e principalmente o espectro de entrada e as atividades de saturação por núcleo alvo. 
O espectro de entrada foi obtido através do código HAMMER-TECHNION ${ }^{137 /}$ à nível celular para a célula representativa do núcleo do Reator IPEN/MB-01 em 84 grupos de energia. Utilizou-se para tanto a biblioteca de dados nucleares ENDF/B-V.

É de extrema importância que o valor do espectro de entrada, calculado pelo código HAMMER-TECHNION seja o mais próximo possível à realidade física do problema.

A tabela 3.6 exibe os valores de fluxo obtido através do código Hammer-Techion que foram utilizados na entrada do código SANDBP:

Tabela 3.6. Espectro de Entrada em 84 Grupos de Energia (83 Pontos).

\begin{tabular}{|c|c|c|}
\hline $\begin{array}{c}\text { Pontos de } \\
\text { Energia } \\
\end{array}$ & Energia (MeV) & Fluxo (Unidades Arbitrárias) \\
\hline 1 & $3.1630 \mathrm{E}-10$ & $4.3636 \mathrm{E}+05$ \\
\hline 2 & $1.0753 \mathrm{E}-09$ & $1.9397 \mathrm{E}+06$ \\
\hline 3 & 2.3403E-09 & $4.2155 \mathrm{E}+06$ \\
\hline 4 & 4.1113E-09 & $7.0194 \mathrm{E}+06$ \\
\hline 5 & $6.3883 \mathrm{E}-09$ & $1.0100 \mathrm{E}+07$ \\
\hline 6 & 9.1713E-09 & $1.3174 \mathrm{E}+07$ \\
\hline 7 & $1.2460 \mathrm{E}-08$ & $1.5991 \mathrm{E}+07$ \\
\hline 8 & $1.6255 \mathrm{E}-08$ & $1.8325 \mathrm{E}+07$ \\
\hline 9 & $2.0556 \mathrm{E}-08$ & $2.0049 \mathrm{E}+07$ \\
\hline 10 & $2.5363 \mathrm{E}-08$ & $2.1071 \mathrm{E}+07$ \\
\hline 11 & $3.0676 \mathrm{E}-08$ & $2.1358 \mathrm{E}+07$ \\
\hline 12 & $3.6495 \mathrm{E}-08$ & $2.0986 \mathrm{E}+07$ \\
\hline 13 & $4.2820 \mathrm{E}-08$ & $2.0039 \mathrm{E}+07$ \\
\hline 14 & 4.9651E-08 & $1.8635 \mathrm{E}+07$ \\
\hline 15 & $5.6988 \mathrm{E}-08$ & $1.6903 \mathrm{E}+07$ \\
\hline 16 & $6.5250 \mathrm{E}-08$ & $1.4881 \mathrm{E}+07$ \\
\hline 17 & 7.4939E-08 & $1.2625 \mathrm{E}+07$ \\
\hline 18 & 8.6229E-08 & $1.0305 \mathrm{E}+07$ \\
\hline 19 & $9.9310 \mathrm{E}-08$ & $8.0901 \mathrm{E}+06$ \\
\hline 20 & $1.1411 \mathrm{E}-07$ & $6.1655 \mathrm{E}+06$ \\
\hline 21 & $1.3140 \mathrm{E}-07$ & $4.5848 \mathrm{E}+06$ \\
\hline 22 & $1.5271 \mathrm{E}-07$ & $3.3241 \mathrm{E}+06$ \\
\hline 23 & 1.7932E-07 & $2.3894 \mathrm{E}+06$ \\
\hline
\end{tabular}




\begin{tabular}{|c|c|c|}
\hline 24 & $2.1282 \mathrm{E}-07$ & $1.7399 \mathrm{E}+06$ \\
\hline 25 & 2.5519E-07 & $1.3248 \mathrm{E}+06$ \\
\hline 26 & 3.0889E-07 & $1.0379 \mathrm{E}+06$ \\
\hline 27 & $3.7695 \mathrm{E}-07$ & $8.4207 \mathrm{E}+05$ \\
\hline 28 & 4.6343E-07 & $6.7637 \mathrm{E}+05$ \\
\hline 29 & $5.6800 \mathrm{E}-07$ & $5.4621 \mathrm{E}+05$ \\
\hline 30 & $7.2925 \mathrm{E}-07$ & $4.3254 \mathrm{E}+05$ \\
\hline 31 & $9.7954 \mathrm{E}-07$ & $3.1205 \mathrm{E}+05$ \\
\hline 32 & $1.2825 \mathrm{E}-06$ & $2.3193 \mathrm{E}+05$ \\
\hline 33 & $1.6475 \mathrm{E}-06$ & $1.8103 \mathrm{E}+05$ \\
\hline 34 & 2.1189E-06 & $1.3883 \mathrm{E}+05$ \\
\hline 35 & $2.7207 \mathrm{E}-06$ & $1.0754 \mathrm{E}+05$ \\
\hline 36 & $3.4935 \mathrm{E}-06$ & $8.2660 \mathrm{E}+04$ \\
\hline 37 & 4.4857E-06 & $6.4592 \mathrm{E}+04$ \\
\hline 38 & $5.7598 \mathrm{E}-06$ & $4.9360 \mathrm{E}+04$ \\
\hline 39 & 7.3957E-06 & $3.3981 \mathrm{E}+04$ \\
\hline 40 & $9.4962 \mathrm{E}-06$ & $3.1152 \mathrm{E}+04$ \\
\hline 41 & 1.2194E-05 & $2.4571 \mathrm{E}+04$ \\
\hline 42 & $1.5657 \mathrm{E}-05$ & $1.9360 \mathrm{E}+04$ \\
\hline 43 & $2.0103 \mathrm{E}-05$ & $1.4123 \mathrm{E}+04$ \\
\hline 44 & $2.5813 \mathrm{E}-05$ & $1.2074 \mathrm{E}+04$ \\
\hline 45 & $3.3145 \mathrm{E}-05$ & $8.9860 \mathrm{E}+03$ \\
\hline 46 & $4.2559 \mathrm{E}-05$ & $7.5721 \mathrm{E}+03$ \\
\hline 47 & $5.4647 \mathrm{E}-05$ & $5.8878 \mathrm{E}+03$ \\
\hline 48 & $7.0168 \mathrm{E}-05$ & $4.5773 \mathrm{E}+03$ \\
\hline 49 & $9.0097 \mathrm{E}-05$ & $3.6173 \mathrm{E}+03$ \\
\hline 50 & $1.1569 \mathrm{E}-04$ & $2.8058 \mathrm{E}+03$ \\
\hline 51 & $1.4855 \mathrm{E}-04$ & $2.2440 \mathrm{E}+03$ \\
\hline 52 & $2.2119 \mathrm{E}-04$ & $1.5187 \mathrm{E}+03$ \\
\hline 53 & $3.6468 \mathrm{E}-04$ & $9.3775 \mathrm{E}+02$ \\
\hline 54 & $6.0126 \mathrm{E}-04$ & $5.7456 \mathrm{E}+02$ \\
\hline 55 & $9.9131 \mathrm{E}-04$ & $3.5312 \mathrm{E}+02$ \\
\hline 56 & $1.6344 \mathrm{E}-03$ & $2.1777 \mathrm{E}+02$ \\
\hline 57 & $2.6947 \mathrm{E}-03$ & $1.3435 \mathrm{E}+02$ \\
\hline 58 & 4.4427E-03 & $8.2925 \mathrm{E}+01$ \\
\hline 59 & $7.3250 \mathrm{E}-03$ & $5.1309 \mathrm{E}+01$ \\
\hline 60 & $1.2076 \mathrm{E}-02$ & $3.2351 \mathrm{E}+01$ \\
\hline 61 & $1.9911 \mathrm{E}-02$ & $2.0721 \mathrm{E}+01$ \\
\hline 62 & $3.2828 \mathrm{E}-02$ & $1.3352 \mathrm{E}+01$ \\
\hline 63 & 5.4123E-02 & $9.0580 \mathrm{E}+00$ \\
\hline
\end{tabular}




\begin{tabular}{|c|c|c|}
\hline 64 & 7.6948E-02 & $7.1147 \mathrm{E}+00$ \\
\hline 65 & $9.8803 \mathrm{E}-02$ & $6.0392 \mathrm{E}+00$ \\
\hline 66 & $1.2687 \mathrm{E}-01$ & $5.2084 \mathrm{E}+00$ \\
\hline 67 & $1.6290 \mathrm{E}-01$ & $4.5260 \mathrm{E}+00$ \\
\hline 68 & $2.0917 \mathrm{E}-01$ & $3.9963 \mathrm{E}+00$ \\
\hline 69 & $2.6858 \mathrm{E}-01$ & $3.6356 \mathrm{E}+00$ \\
\hline 70 & $3.4486 \mathrm{E}-01$ & $3.2164 \mathrm{E}+00$ \\
\hline 71 & 4.4281E-01 & $2.5268 \mathrm{E}+00$ \\
\hline 72 & $5.6858 \mathrm{E}-01$ & $2.3802 \mathrm{E}+00$ \\
\hline 73 & 7.3007E-01 & $2.1938 \mathrm{E}+00$ \\
\hline 74 & 9.3743E-01 & $1.6038 \mathrm{E}+00$ \\
\hline 75 & $1.2037 \mathrm{E}-00$ & $1.3561 \mathrm{E}+00$ \\
\hline 76 & $1.5455 \mathrm{E}-00$ & $1.1446 \mathrm{E}+00$ \\
\hline 77 & $1.9845 \mathrm{E}-00$ & 8.3558E-01 \\
\hline 78 & $2.5482 \mathrm{E}-00$ & $6.8713 \mathrm{E}-01$ \\
\hline 79 & $3.2710 \mathrm{E}-00$ & $3.9485 \mathrm{E}-01$ \\
\hline 80 & 4.2012E-00 & 2.2192E-01 \\
\hline 81 & 5.3945E-00 & 1.0888E-01 \\
\hline 82 & $6.9266 \mathrm{E}-00$ & 4.0965E-02 \\
\hline 83 & 8.8940E-00 & $1.3190 \mathrm{E}-02$ \\
\hline
\end{tabular}

As figuras 3.5 e 3.6 mostram graficamente o espectro de entrada para os valores de fluxo exibidos na tabela 3.6, em pontos e grupos de energia respectivamente: 


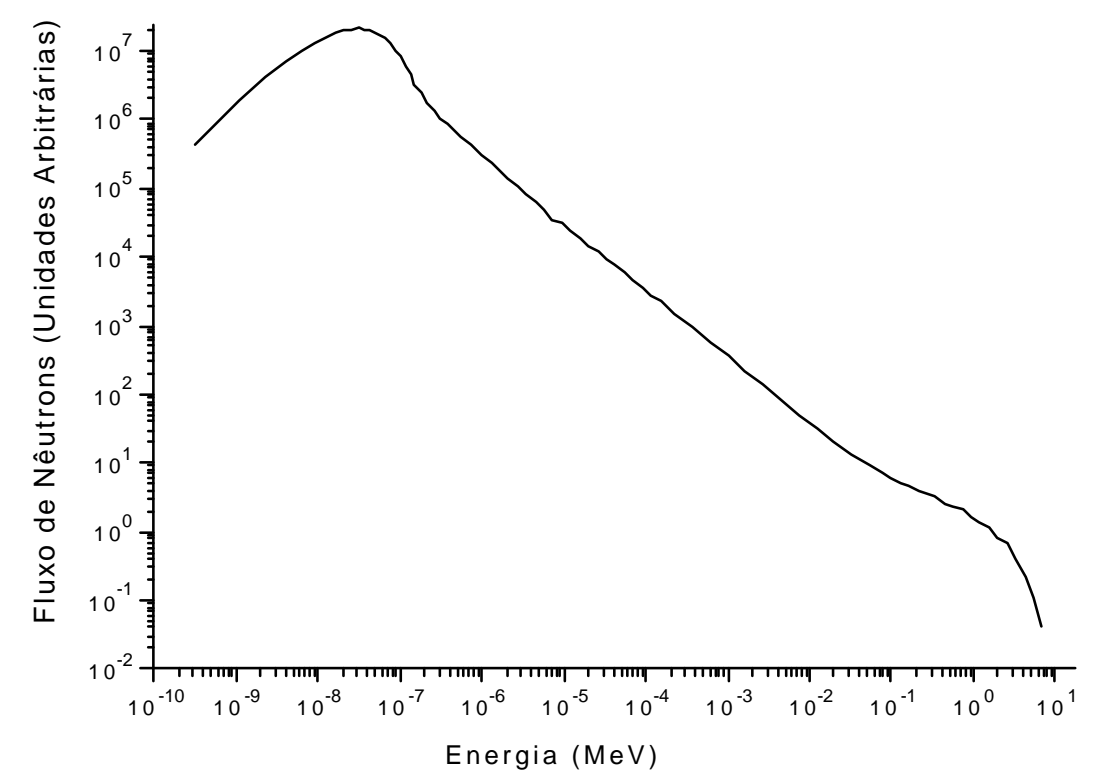

Figura 3.5 - Espectro de entrada obtido via cálculo em 83 pontos de energia

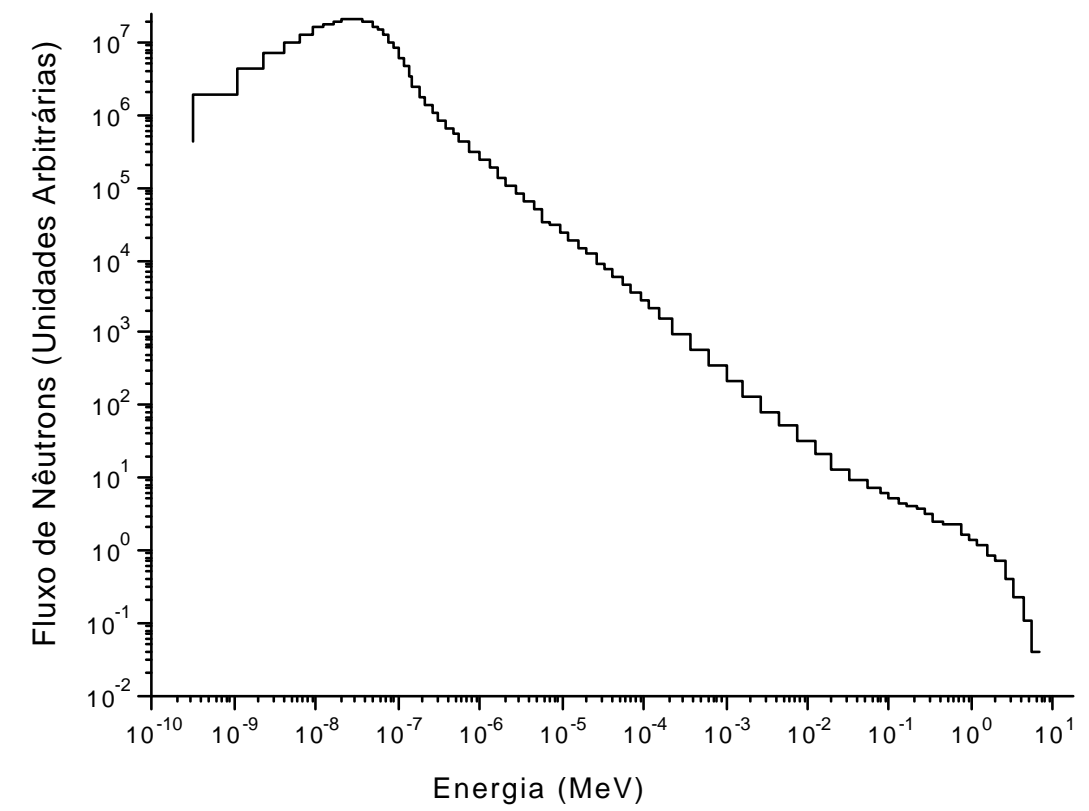

Figura 3.6 - Espectro de entrada obtido via cálculo em 84 grupos de energia 
Para determinar a atividade de saturação por núcleo alvo da amostra (folha de ativação) irradiada, é necessário conhecer o número de núcleos alvos presentes na mostra, que é dado por:

$$
N_{A}=\frac{N_{0}}{\mathrm{~A}} m f_{i s o}
$$

Sendo,

$$
\begin{aligned}
& N_{0} \text { o número de Avogrado, } \\
& m \quad \text { a massa da folha, } \\
& f_{i s o} \quad \text { a fração isotópica do material irradiado, }
\end{aligned}
$$

A o peso atômico.

Dividindo a equação (2.12) pela equação (3.8) e introduzindo-se os fatores de correção $F r$ e $F n$ que se referem a fatores de correção devido a rampa de subida de potência durante a irradiação das folhas e ao fator de normalização monitorado pelo canal 10 da instrumentação nuclear, obtém-se a atividade de saturação por núcleo-alvo:

$$
\frac{A^{\infty}}{N_{A}}=\frac{(C-B G) e^{\lambda t_{e}}}{I \varepsilon_{c}\left(1-e^{-\lambda t_{i}}\right)} \frac{\mathrm{A}}{N_{0} m f_{\text {iso }}} \frac{F r}{F n}
$$

Uma descrição mais detalhada das configurações dos parâmetros de entrada, bem como o conjunto de dados de entrada utilizado neste trabalho, podem ser vistos no Anexo B.

\subsection{Análise das Incertezas Experimentais}

O cálculo das incertezas das atividades de saturação das folhas irradiadas pode ser determinado aplicando-se o método matemático de propagação de erros na equação (2.12):

$$
\frac{\delta A^{\infty}}{A^{\infty}}=\sqrt{\left(\frac{\delta \lambda}{\lambda}\right)^{2}+\left(\frac{\delta(C-B G)}{C-B G}\right)^{2}+\left(\frac{\delta\left(e^{\lambda t_{e}}\right)}{e^{\lambda t_{e}}}\right)^{2}+\left(\frac{\delta \varepsilon}{\varepsilon}\right)^{2}+\left(\frac{\delta}{I}\right)^{2}+\left(\frac{\delta\left(1-e^{-\lambda t}\right)}{1-e^{-\lambda t}}\right)^{2}+\left(\frac{\delta\left(1-e^{-\lambda t_{c}}\right)}{1-e^{-\lambda t_{c}}}\right)^{2}}
$$


A fim de simplificar o cálculo da incerteza, pode-se utilizar o mesmo raciocínio descrito no item 3.3.1, expandindo em série de Taylor o termo $\left(1-e^{-\lambda t_{c}}\right)$ da equação (2.12), e por praticidade faremos o termo $(C-B G)=C$, que é o número de contagens líquidas, já descontada a radiação de fundo (background), sendo assim a equação (2.12) pode ser reescrita da seguinte forma:

$$
A^{\infty}=\frac{C^{\prime} e^{\lambda t_{e}}}{\varepsilon \cdot I \cdot t_{c}\left(1-e^{-\lambda t}\right)}
$$

Após as simplificações, a incerteza da atividade de saturação pode ser expressa da seguinte forma:

$$
\frac{\delta A^{\infty}}{A^{\infty}}=\sqrt{\left(\frac{\delta C^{\prime}}{C^{\prime}}\right)^{2}+\left(\frac{\delta\left(e^{\lambda t_{e}}\right)}{e^{\lambda t_{e}}}\right)^{2}+\left(\frac{\delta \varepsilon}{\varepsilon}\right)^{2}+\left(\frac{\delta I}{I}\right)^{2}+\left(\frac{\delta\left(1-e^{-\lambda t}\right)}{1-e^{-\lambda t}}\right)^{2}+\left(\frac{\delta\left(t_{c}\right)}{t_{c}}\right)^{2}}
$$

Os erros percentuais relativos aos tempos são estimados como sendo a metade da menor divisão dos cronômetros. Assim,

$$
\left(\frac{\boldsymbol{\delta}\left(e^{\lambda t_{e}}\right)}{e^{\lambda t_{e}}}\right)^{2},\left(\frac{\boldsymbol{\delta}\left(1-e^{-\lambda t}\right)}{1-e^{-\lambda t}}\right)^{2},\left(\frac{\boldsymbol{\delta}\left(t_{c}\right)}{t_{c}}\right)^{2}<<0,1 \%
$$

Considerando que os erros percentuais relativos aos tempos são menores que $0,1 \%$, estes são desprezados. Desta forma a equação (3.11) passa a ser escrita:

$$
\frac{\delta A^{\infty}}{A^{\infty}}=\sqrt{\left(\frac{\delta C^{\prime}}{C^{\prime}}\right)^{2}+\left(\frac{\delta \varepsilon}{\varepsilon}\right)^{2}+\left(\frac{\delta I}{I}\right)^{2}}
$$

A equação (3.12) é facilmente resolvida, considerando que a taxa de contagens líquidas, sua incerteza, bem como a eficiência e incerteza, são obtidos experimentalmente, através da espectrometria gama e a abundância gama e sua incerteza associada são obtidas através de bibliotecas de dados. 
O cálculo da incerteza da atividade de saturação por núcleo alvo, é realizado através do mesmo procedimento, ou seja, neste caso diferenciando a equação (3.9) em relação aos termos que compõe sua fórmula, para isso pode-se escrevê-la:

$$
\frac{A^{\infty}}{N_{A}}=\frac{A^{\infty}}{m} K
$$

Onde $K$ é uma constante. Assim diferencia-se a equação (3.13):

$$
\delta\left(\frac{A^{\infty}}{N_{A}}\right)=\left(\frac{A^{\infty}}{N_{A}}\right) \sqrt{\left(\frac{\delta A^{\infty}}{A^{\infty}}\right)^{2}+\left(\frac{\delta m}{m}\right)^{2}}
$$




\section{UTILIZAÇÃO DO CÓDIGO SANDBP ${ }^{138 /}$}

\subsection{Introdução}

O código computacional SANDBP foi desenvolvido no Instituto de Técnicas Nucleares da Universidade Técnica de Budapeste e é uma versão modificada e aperfeiçoada do código SAND-II. O código SANDBP pode ser usado para determinar o espectro de nêutrons pelo método de ativação por folhas. O código faz uma iteração de ajuste, iniciando o processo a partir de um espectro de entrada (inicial). A solução apropriada do espectro será atingida após certo número de iterações, quando as atividades de saturação calculadas comparadas às medidas estiverem apresentando certos desvios especificados na entrada do código. A faixa de energia da solução do espectro é representada por no máximo 640 intervalos de energia e pode-se incluir a possibilidade de calcular a atenuação de fluxo das folhas cobertas com materiais como cádmio, boro e ouro. O código também calcula diferentes funções resposta da solução do espectro e também permite uma análise das incertezas através do método de Monte Carlo, fornecendo a matriz covariância da solução do espectro de nêutrons pode ser encontrada ${ }^{138 /}$.

\subsection{Procedimento Matemático}

O código SANDBP utiliza um algoritmo de ajuste iterativo, dado por:

$$
\Phi_{j}^{k+1}=\Phi_{j}^{k} e^{C_{j}^{k}} \quad \mathrm{j}=1, \ldots, \mathrm{m}
$$

Sendo:

$$
C_{j}^{k}=\frac{\sum_{i=1}^{n} W_{i j}^{k} \ln \left(\frac{A_{i}}{A_{i}^{k}}\right)}{\sum_{i=1}^{n} W_{i j}^{k}}
$$


Onde,

$\Phi_{j}^{k} \quad$ é o espectro de nêutrons na k-ésima iteração, no j-ésimo intervalo de energia,

$C_{j}^{k} \quad$ é o termo de correção de fluxo na k-ésima iteração, para o j-ésimo grupo,

$A_{i}^{k} \quad$ é a atividade de saturação calculada para o i-ésimo detector, na k-ésima iteração do espectro,

$m$ é o número de intervalos de energia,

$n \quad$ é o número de detectores utilizados.

O termo $W_{i j}^{k}$ é a função peso, que leva em conta as incertezas das atividades e seções de choque, e é expressa matematicamente por:

$$
\begin{gathered}
W_{i j}^{k}=\frac{A_{i j}^{k}}{A_{i}^{k} F_{i j}} \\
A_{i j}^{k}=\Phi_{j}^{k} \sigma_{i j}\left[\left(E_{j+1}\right)-\left(E_{j}\right)\right] \\
A_{i}^{k}=\sum_{j=1}^{m} A_{i j}^{k} \\
F_{i j}=\left[\left(\delta A_{i}\right)^{n}\left(\delta \sigma_{i j}\right)^{l}\right]
\end{gathered}
$$

Onde,

$\sigma_{i j} \quad$ é a seção de choque do i-ésimo detector medida no j-ésimo intervalo de energia

$\delta A_{i} \quad$ é o desvio padrão do valor da atividade de saturação,

$\delta \sigma_{i j} \quad$ é o desvio padrão do valor da seção de choque,

l e $n$ são índices da função peso $F_{i j}$, e cada um deles pode assumir os valores 0,1 ou 2 .

O desvio padrão dos valores da atividade medida para calculada são obtidos após cada iteração e expressos em porcentagem, conforme segue: 


$$
D E V_{\%}^{k}=\left[\sum_{i=1}^{N} R D_{i}^{2}-\frac{\left(\sum_{i=1}^{N} R D_{i}^{2}\right)^{2}}{N}\right] \frac{100}{N-1}, \quad R D_{i}=\frac{A_{i m}-A_{i c}^{k}}{A_{i c}^{k}}
$$

Sendo,
$A_{i c}^{k} \quad$ a atividade de saturação calculada para a i-ésima folha detectora na k- ésima iteração,
$A_{i m} \quad$ a atividade medida no i-ésimo detector.

Para mensurar as incertezas dos dados, o termo $D E V$ é expresso em intervalos de confiança, utilizando a seguinte substituição:

$$
C D_{i}=\frac{R D_{i}}{\delta A_{i m}}
$$

Desta forma é possível levar em conta ("pesar")o desvio-padrão da atividade de saturação por núcleo alvo medida

A iteração é interrompida quando o valor do desvio padrão for menor que o valor especificado na entrada do código. É possível ainda introduzir outros parâmetros de controle, como o número máximo de iterações.

O código utiliza ainda o método de análise de incertezas Monte Carlo para calcular a matriz covariância da solução do espectro. Neste cálculo, os valores das atividades de saturação por núcleo alvo especificadas na entrada do código, bem como o espectro de entrada e as seções de choque são modificados, assumindo-se que estas quantidades seguem uma distribuição normal, dada por:

$$
A_{i}^{r}=A_{i}+g \delta A_{i} \quad \mathrm{i}=1, \ldots, \mathrm{n}
$$

Onde,

$A_{i}^{r} \quad$ é o r-ésimo valor da i-ésima atividade $A_{i}$ medida,

$\delta A_{i} \quad$ é o desvio padrão da i-ésima atividade, 
g é o número da distribuição normal padrão.

$$
\Phi_{j}^{r}=\Phi_{j}+g \delta \Phi_{j} \quad \mathrm{j}=1, . ., \mathrm{m}
$$

Onde,

$\Phi_{j}^{r} \quad$ é o r-ésimo valor do j-ésimo grupo,

$\delta \Phi_{j} \quad$ é o desvio padrão de $\Phi_{j}$.

$$
\sigma_{i j}^{r}=\sigma_{i j}+g \delta \sigma_{i j} \quad \mathrm{i}=1, . ., \mathrm{n} ; \mathrm{j}=1, . ., \mathrm{m}
$$

Onde,

$\sigma_{i j}^{r} \quad$ é o r-ésimo valor da i-ésima reação da seção de choque, para o j-ésimo grupo de energia ,

$\delta \sigma_{i j} \quad$ é o desvio padrão de $\sigma_{i j}$.

O código calcula então a covariância da solução do espectro, que é dada por:

$$
S_{j i}=\frac{\sum_{r=1}^{k} \Phi_{j}^{r} \Phi_{i}^{r}}{K}-\frac{\left(\sum_{r=1}^{k} \Phi_{j}^{r}\right)\left(\sum_{r=1}^{k} \Phi_{i}^{r}\right)}{K^{2}}
$$

Sendo,

$S_{j i} \quad$ a covariância entre os valores do espectro,

$\Phi_{j} \quad$ o espectro do j-ésimo grupo,

$\Phi_{i} \quad$ o espectro do j-ésimo grupo,

$r \quad$ o resultado mostrado na r-ésima execução do Monte Carlo,

$K \quad$ o número de execuções.

O código possibilita ainda a execução do método de Monte Carlo para calcular a chamada "A melhor das soluções"(GOS - Goodness of the solution), ou seja, os melhores 
valores para cada grupo de energia. Este resultado mostra o quanto a solução do espectro depende do espectro de entrada.

O cálculo destes valores significa uma análise sensitiva especial que determina a sensitividade dos coeficientes do espectro de saída para o de entrada. O código varia somente o espectro de entrada e dos dados resultantes calcula os valores "GOS", que são dados por:

$$
G O S=\frac{d \Phi_{o u t}}{d \Phi_{i n}}
$$

O código oferece diferentes saídas dos resultados (outputs) do ajuste do espectro de nêutrons e análise das incertezas, como exemplo: Tabelas resumindo os resultados das iterações intermediária e final, a solução do espectro em diferentes grupos de energia, a covariância, matriz correlação e os valores "GOS"

Comparado com outros códigos existentes para ajuste de espectro de nêutrons e análise de incertezas o SANDBP apresenta certas vantagens e desvantagens. Uma importante vantagem é o fato de que o algoritmo iterativo é baseado no método dos mínimos quadrados. O código oferece ainda uma conversão rápida para a solução e tem-se menor esforço na preparação dos dados de entrada (input). Outros códigos que são baseados no método dos mínimos quadrados, como o (STAY'SL ${ }^{132 /}$ ) por exemplo, requerem uma inversão da matriz e mais tempo de cálculo, além de exigirem informações completas da covariância na entrada do código. O código SANDBP não requer uma informação da covariância na entrada para o processo de ajuste. Outra vantagem é a possibilidade de pesar os valores das incertezas das medidas de taxa de reação e seção de choque no processo de iteração; Desta forma o código apresenta uma maior precisão nos dados de entrada. A principal desvantagem é o fato do código não apresentar um valor exato como resultado, fornecendo dados de entrada que nem sempre conduzirão ao mesmo espectro de saída. Este não é o caso de códigos que levam em conta todas as informações da covariância nos dados de entrada. 


\section{RESULTADOS EXPERIMENTAIS}

Os valores das atividades de saturação por núcleo-alvo determinadas experimentalmente e suas incertezas podem ser visualizados na tabela 5.1. Neles estão corrigidos os fatores de rampa, normalização e auto-absorção, bem como os fatores de perturbação de fluxo devido ao efeito de auto-blindagem.

Tabela 5.1-Atividades de Saturação por Núcleo Alvo Determinadas Experimentalmente.

\begin{tabular}{|c|c|}
\hline Folhas & $\begin{array}{l}\text { Atividade de Saturação por Núcleo } \\
\text { Alvo medida (Bq/núcleo alvo) }\end{array}$ \\
\hline $\mathrm{Au}^{197}$ & $2,8680 \cdot 10^{-13} \pm 1,8527 \cdot 10^{-14}$ \\
\hline $\mathrm{Au}^{197 *}$ & $1,9644.10^{-13} \pm 1,2690.10^{-14}$ \\
\hline $\mathrm{Sc}^{45}$ & $1,9445.10^{-14} \pm 6,3079.10^{-16}$ \\
\hline $\mathrm{Sc}^{45 *}$ & $1,4696.10^{-15} \pm 2,0279.10^{-17}$ \\
\hline $\mathrm{U}^{238}$ & $2,6702 \cdot 10^{-14} \pm 2,0480 \cdot 10^{-15}$ \\
\hline $\mathrm{U}^{238 *}$ & $2,7236.10^{-14} \pm 2,0890.10^{-15}$ \\
\hline $\mathrm{Ti}^{48 *}$ & $4,1608 \cdot 10^{-19} \pm 1,9805 \cdot 10^{-20}$ \\
\hline $\mathrm{Ti}^{47}$ & $3,9937.10^{-17} \pm 5,1599 \cdot 10^{-18}$ \\
\hline $\mathrm{Ti}^{47} *$ & $3,3070.10^{-17} \pm 4,2726.10^{-18}$ \\
\hline $\mathrm{Ni}^{58}$ & $2,0113 \cdot 10^{-16} \pm 7,8843.10^{-18}$ \\
\hline $\mathrm{Ni}^{58 *}$ & $1,9710.10^{-16} \pm 7,7263.10^{-18}$ \\
\hline $\operatorname{In}^{115}$ & $3,3111.10^{-16} \pm 1,1122.10^{-17}$ \\
\hline $\operatorname{In}^{115 *}$ & $3,5701.10^{-16} \pm 2,5904.10^{-17}$ \\
\hline $\mathrm{Mg}^{24}$ & $2,2768.10^{-18} \pm 1,1657.10^{-19}$ \\
\hline $\mathrm{Mg}^{24 *}$ & $2,0773 \cdot 10^{-18} \pm 6,4189 \cdot 10^{-20}$ \\
\hline $\mathrm{Al}^{27} *$ & $1,2514.10^{-18} \pm 1,0061.10^{-19}$ \\
\hline
\end{tabular}

*Folhas cobertas com cádmio

Os valores de desvio padrão das atividades de saturação por núcleo alvo medidas, obtidos através da equação (3.14), devem ser expressos em porcentagem, a fim de se adequarem às configurações exigidas na entrada do código SANDBP, conforme tabela 5.2. 
Tabela 5.2 - Atividades de saturação por núcleo alvo determinadas experimentalmente e suas incertezas expressas em porcentagem.

\begin{tabular}{|c|c|c|}
\hline Folhas & $\frac{A^{\infty}}{N_{A}}($ Bqlátomo $)$ & $\sigma\left(\frac{A^{\infty}}{N_{A}}\right)(\%)$ \\
\hline $\mathrm{Au}^{197}$ & $2,8680.10^{-13}$ & 6,460 \\
\hline $\mathrm{Au}^{197 *}$ & $1,9644.10^{-13}$ & 6,460 \\
\hline $\mathrm{Sc}^{45}$ & $1,9445.10^{-14}$ & 3,244 \\
\hline $\mathrm{Sc}^{45 *}$ & $1,4696.10^{-15}$ & 1,380 \\
\hline $\mathrm{U}^{238}$ & $2,6702.10^{-14}$ & 7,670 \\
\hline $\mathrm{U}^{23 * *}$ & $2,7236.10^{-14}$ & 7,670 \\
\hline $\mathrm{Ti}^{48 *}$ & $4,1608.10^{-19}$ & 4,760 \\
\hline $\mathrm{Ti}^{47}$ & $3,9937.10^{-17}$ & 12,92 \\
\hline $\mathrm{Ti}^{47 *}$ & $3,3070.10^{-17}$ & 12,92 \\
\hline $\mathrm{Ni}^{58}$ & $2,0113.10^{-16}$ & 3,920 \\
\hline $\mathrm{Ni}^{58 *}$ & $1,9710.10^{-16}$ & 3,920 \\
\hline $\mathrm{In}^{115}$ & $3,3111.10^{-16}$ & 3,359 \\
\hline $\mathrm{In}^{115 *}$ & $3,5701.10^{-16}$ & 7,256 \\
\hline $\mathrm{Mg}^{24}$ & $2,2768.10^{-18}$ & 5,120 \\
\hline $\mathrm{Mg}^{24 *}$ & $2,0773.10^{-18}$ & 3,090 \\
\hline $\mathrm{Al}^{27 *}$ & $1,2514.10^{-18}$ & 8,040 \\
\hline
\end{tabular}

*Folhas cobertas com cádmio

As Tabelas e figuras a seguir mostram o espectro de energia dos nêutrons do Reator IPEN/MB-01, obtidos experimentalmente através do código SANDBP após três ajustes iterativos, utilizando 16 detectores de ativação: $\mathrm{Au}^{197}, \mathrm{Au}^{197} \mathrm{c} / \mathrm{cd}, \mathrm{Sc}^{45}, \mathrm{Sc}^{45} \mathrm{c} / \mathrm{cd}, \mathrm{U}^{238}$, $\mathrm{U}^{238} \mathrm{c} / \mathrm{cd}, \mathrm{In}^{115}, \mathrm{In}^{115} \mathrm{c} / \mathrm{cd}, \mathrm{Mg}^{24}, \mathrm{Mg}^{24} \mathrm{c} / \mathrm{cd}, \mathrm{Al}^{27} \mathrm{c} / \mathrm{cd}, \mathrm{Ni}^{58} \mathrm{c} / \mathrm{cd}, \mathrm{Ni}^{58}$ e $\mathrm{Ti}^{48} \mathrm{c} / \mathrm{cd}, \mathrm{Ti}^{47}$ e $\mathrm{Ti}^{47} \mathrm{c} / \mathrm{cd}$. 
Tabela 5.3 - Valores Absolutos de Fluxo Diferencial e Integral obtidos em 51 pontos.

\begin{tabular}{|c|c|c|}
\hline Energia $(\mathrm{MeV})$ & $\begin{array}{c}\text { Fluxo Diferencial } \\
\left(\mathrm{n} / \mathrm{cm}^{2} \mathrm{~s} M e V\right)\end{array}$ & Fluxo Integral $\left(\mathrm{n} / \mathrm{cm}^{2} \mathrm{~s}\right)$ \\
\hline $1 \mathrm{E}-10$ & $3,958 \mathrm{E} 14$ & $5,118 \mathrm{E} 9$ \\
\hline $1 \mathrm{E}-9$ & $3,82 \mathrm{E} 15$ & $5,117 \mathrm{E} 9$ \\
\hline $1 \mathrm{E}-8$ & $8,423 \mathrm{E} 15$ & $5,083 \mathrm{E} 9$ \\
\hline $2,8 \mathrm{E}-8$ & $7,369 \mathrm{E} 15$ & 4,931E9 \\
\hline $7,6 \mathrm{E}-8$ & $4,063 \mathrm{E} 15$ & $4,577 \mathrm{E} 9$ \\
\hline $1,15 \mathrm{E}-7$ & $1,203 \mathrm{E} 15$ & 4,419E9 \\
\hline $2,55 \mathrm{E}-7$ & $3,441 \mathrm{E} 14$ & $4,251 \mathrm{E} 9$ \\
\hline $5,5 \mathrm{E}-7$ & $1,425 \mathrm{E} 14$ & $4,149 \mathrm{E} 9$ \\
\hline $1,275 \mathrm{E}-6$ & $6,403 \mathrm{E} 13$ & $4,046 \mathrm{E} 9$ \\
\hline $2,8 \mathrm{E}-6$ & $2,727 \mathrm{E} 13$ & $3,948 \mathrm{E} 9$ \\
\hline $6,3 \mathrm{E}-6$ & $1,255 \mathrm{E} 13$ & $3,853 \mathrm{E} 9$ \\
\hline $1,35 \mathrm{E}-5$ & $5,277 \mathrm{E} 12$ & $3,762 \mathrm{E} 9$ \\
\hline $3 \mathrm{E}-5$ & $2,433 \mathrm{E} 12$ & $3,675 \mathrm{E} 9$ \\
\hline $6,9 \mathrm{E}-5$ & $1,219 \mathrm{E} 12$ & $3,58 \mathrm{E} 9$ \\
\hline $1,35 \mathrm{E}-4$ & $7,292 \mathrm{E} 11$ & $3,5 \mathrm{E} 9$ \\
\hline $2,2 \mathrm{E}-4$ & $4,671 \mathrm{E} 11$ & $3,438 \mathrm{E} 9$ \\
\hline $3,6 \mathrm{E}-4$ & $2,966 \mathrm{E} 11$ & $3,373 \mathrm{E} 9$ \\
\hline $5,75 \mathrm{E}-4$ & $1,842 \mathrm{E} 11$ & 3,309E9 \\
\hline $9,6 \mathrm{E}-4$ & $1,106 \mathrm{E} 11$ & $3,238 \mathrm{E} 9$ \\
\hline 0,0016 & $6,838 \mathrm{E} 10$ & $3,167 \mathrm{E} 9$ \\
\hline 0,0027 & $4,155 \mathrm{E} 10$ & 3,092E9 \\
\hline 0,0045 & $2,6 \mathrm{E} 10$ & 3,017E9 \\
\hline 0,0072 & $1,633 \mathrm{E} 10$ & $2,947 \mathrm{E} 9$ \\
\hline 0,012 & $1,053 \mathrm{E} 10$ & $2,868 \mathrm{E} 9$ \\
\hline 0,019 & $6,791 \mathrm{E} 9$ & $2,795 \mathrm{E} 9$ \\
\hline 0,032 & $4,457 \mathrm{E} 9$ & $2,706 \mathrm{E} 9$ \\
\hline 0,0525 & $3,08 \mathrm{E} 9$ & $2,615 \mathrm{E} 9$ \\
\hline 0,088 & 2,277E9 & $2,506 \mathrm{E} 9$ \\
\hline 0,135 & $1,831 \mathrm{E} 9$ & 2,399E9 \\
\hline 0,19 & $1,573 \mathrm{E} 9$ & $2,298 \mathrm{E} 9$ \\
\hline 0,255 & $1,408 \mathrm{E} 9$ & $2,196 \mathrm{E} 9$ \\
\hline 0,32 & $1,187 \mathrm{E} 9$ & $2,104 \mathrm{E} 9$ \\
\hline 0,4 & $1,03 \mathrm{E} 9$ & 2,009E9 \\
\hline 0,5 & $1,09 \mathrm{E} 9$ & $1,906 \mathrm{E} 9$ \\
\hline 0,6 & $1,158 \mathrm{E} 9$ & $1,797 \mathrm{E} 9$ \\
\hline 0,72 & $1,046 \mathrm{E} 9$ & $1,658 \mathrm{E} 9$ \\
\hline 0,84 & $8,692 \mathrm{E} 8$ & $1,533 \mathrm{E} 9$ \\
\hline 1 & $7,097 \mathrm{E} 8$ & $1,394 \mathrm{E} 9$ \\
\hline 1,4 & $5,732 \mathrm{E} 8$ & $1,11 \mathrm{E} 9$ \\
\hline 1,8 & $4,657 \mathrm{E} 8$ & $8,806 \mathrm{E} 8$ \\
\hline 2,3 & $3,591 \mathrm{E} 8$ & $6,478 \mathrm{E} 8$ \\
\hline 2,9 & $2,355 \mathrm{E} 8$ & $4,323 \mathrm{E} 8$ \\
\hline 3,7 & $1,434 \mathrm{E} 8$ & $2,439 \mathrm{E} 8$ \\
\hline 4,5 & 7,392E7 & $1,292 \mathrm{E} 8$ \\
\hline 5,5 & $2,676 \mathrm{E} 7$ & $5,528 \mathrm{E} 7$ \\
\hline 6,7 & 9,888E6 & $2,316 \mathrm{E} 7$ \\
\hline 8,2 & 3,337E6 & $8,328 \mathrm{E} 6$ \\
\hline 10 & 782400 & 2,322E6 \\
\hline 12,5 & 96200 & 365500 \\
\hline 16 & 7199 & 28790 \\
\hline 20 & 0 & 0 \\
\hline
\end{tabular}




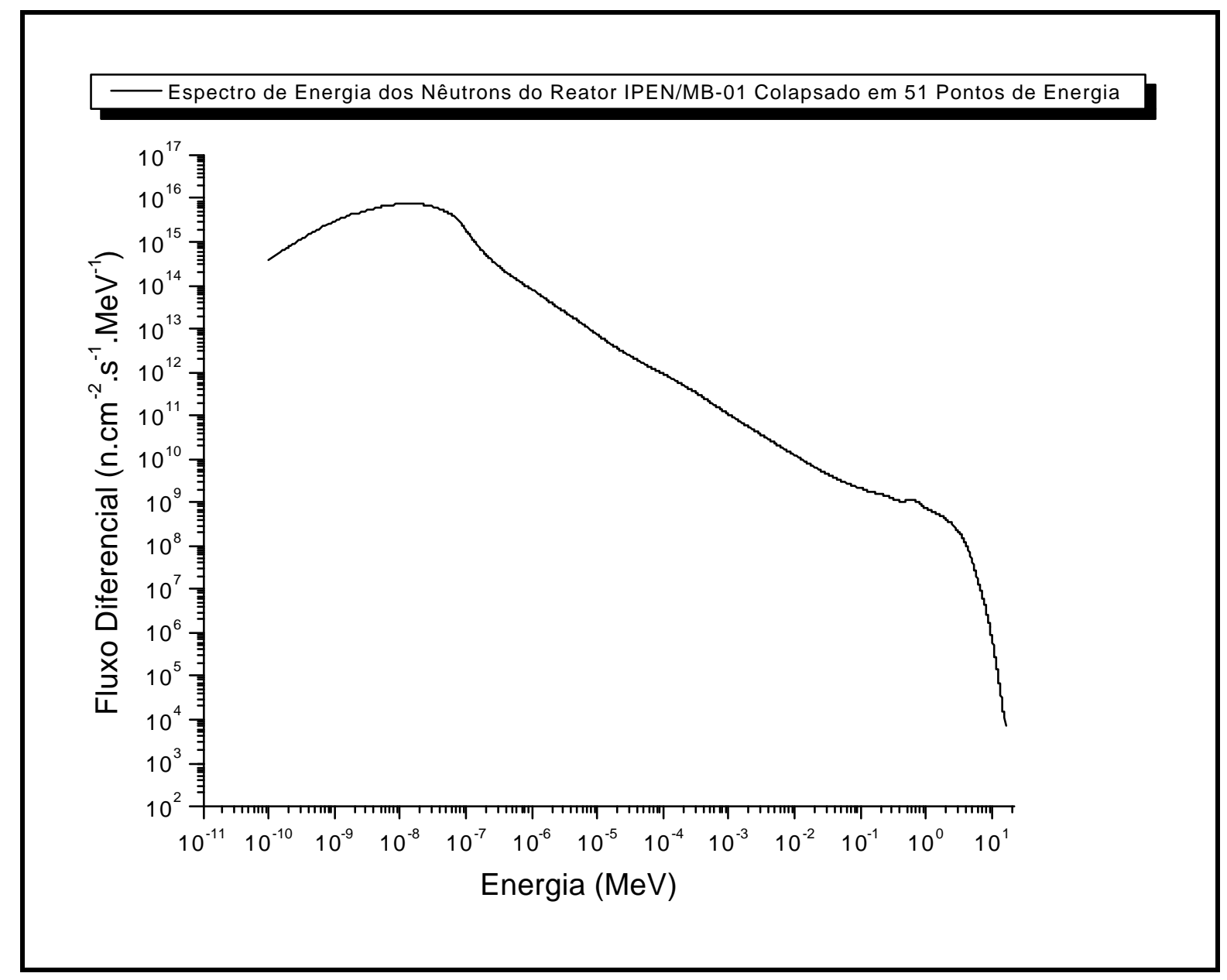

Figura 5.1 - Espectro de Energia dos Nêutrons do Reator IPEN/MB-01 Colapsado em 51 Pontos de Energia. 


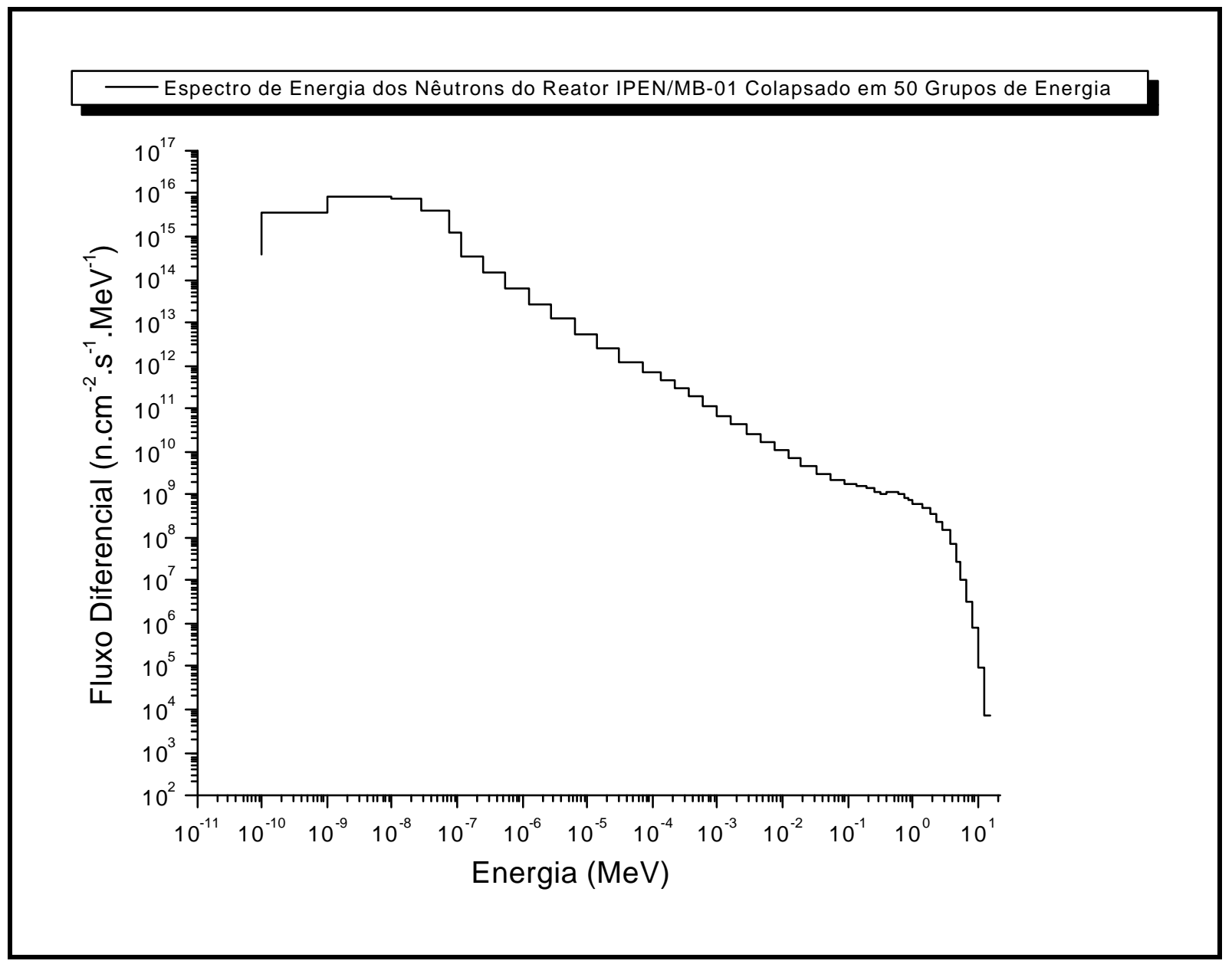

Figura 5.2 - Espectro de Energia dos Nêutrons do Reator IPEN/MB-01 Colapsado em 50 Grupos de Energia, 


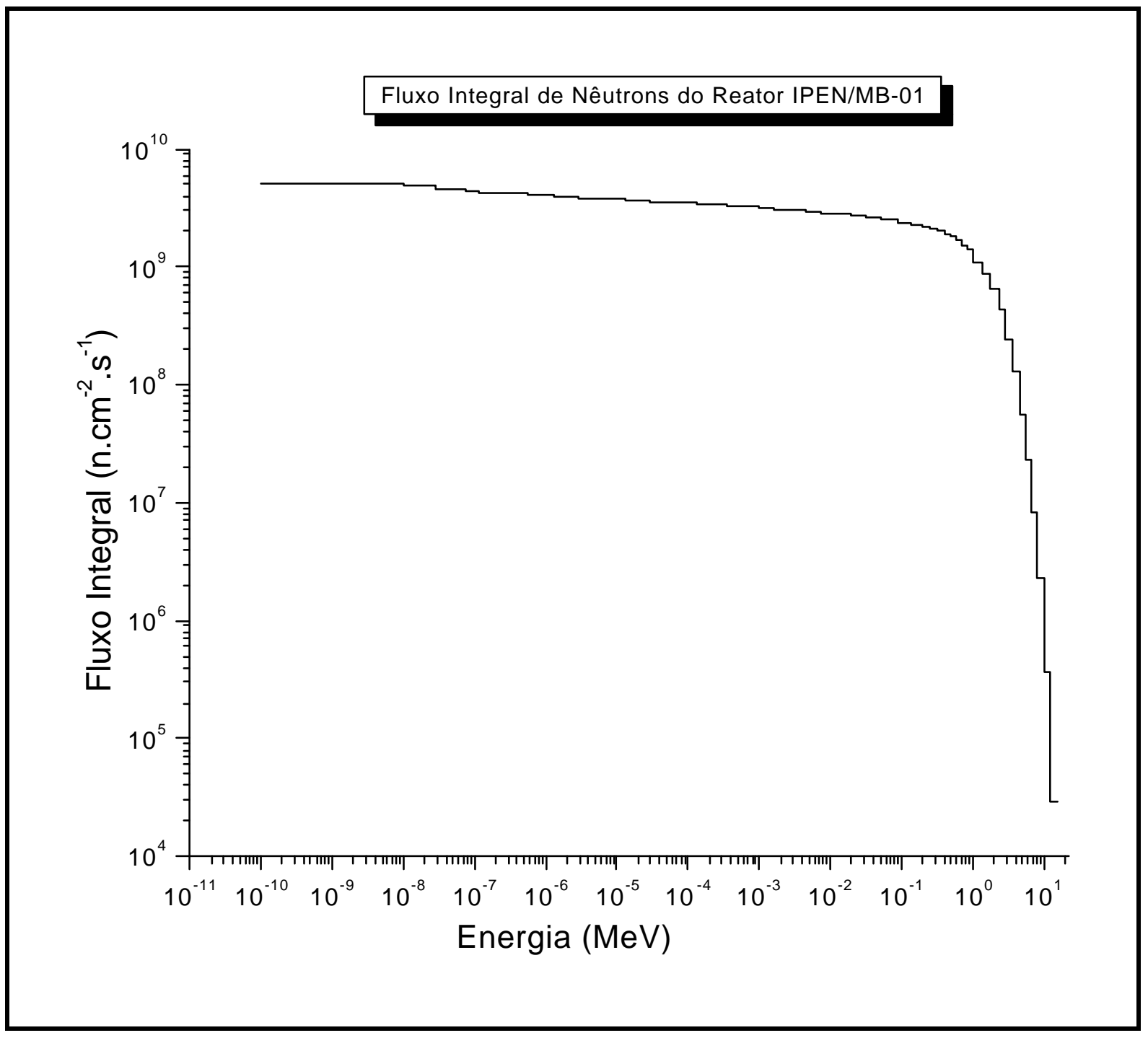

Figura 5.3 - Variação Energética do Fluxo Integral de Nêutrons do Reator IPEN/MB01 em 50 Grupos de Energia. 
Tabela 5.4 - Valores Absolutos de Fluxo Diferencial obtidos em 26 grupos de energia

\begin{tabular}{|c|c|c|}
\hline Grupo & $\begin{array}{c}\text { Energia } \\
(\mathrm{MeV}) \\
\end{array}$ & $\begin{array}{c}\text { Fluxo } \\
\left(\mathrm{n} / \mathrm{cm}^{2} \mathrm{sMeV}\right)\end{array}$ \\
\hline 1 & $1,050.10^{1}$ & $2,658 \cdot 10^{7}$ \\
\hline 2 & $6,500 \cdot 10^{0}$ & $1,724.10^{8}$ \\
\hline 3 & $4,000 \cdot 10^{0}$ & $3,751.10^{8}$ \\
\hline 4 & $2,500.10^{0}$ & $5,34.10^{8}$ \\
\hline 5 & $1,400.10^{0}$ & $4,648 \cdot 10^{8}$ \\
\hline 6 & $8,000.10^{-1}$ & $4,346.10^{8}$ \\
\hline 7 & $4,000.10^{-1}$ & $2,73.10^{8}$ \\
\hline 8 & $2,000 \cdot 10^{-1}$ & $1,961.10^{8}$ \\
\hline 9 & $1,000.10^{-1}$ & $1,634.10^{8}$ \\
\hline 10 & $4,650.10^{-2}$ & $1,359.10^{8}$ \\
\hline 11 & $2,150 \cdot 10^{-2}$ & $1,234.10^{8}$ \\
\hline 12 & $1,000.10^{-2}$ & $1,12.10^{8}$ \\
\hline 13 & $4,650.10^{-2}$ & $1,163.10^{8}$ \\
\hline 14 & $2,150.10^{-3}$ & $1,04.10^{8}$ \\
\hline 15 & $1,000.10^{-3}$ & $1,08.10^{8}$ \\
\hline 16 & $4,650.10^{-4}$ & $1,002.10^{8}$ \\
\hline 17 & $2,150.10^{-4}$ & $1,01.10^{8}$ \\
\hline 18 & $1,000.10^{-4}$ & $9,254.10^{7}$ \\
\hline 19 & $4,650.10^{-5}$ & $8,499.10^{7}$ \\
\hline 20 & $2,150.10^{-5}$ & $8,615 \cdot 10^{7}$ \\
\hline 21 & $1,000.10^{-5}$ & $9,144.10^{7}$ \\
\hline 22 & $4,650.10^{-6}$ & $9,206.10^{7}$ \\
\hline 23 & $2,150.10^{-6}$ & $9,52.10^{7}$ \\
\hline 24 & $1,000.10^{-6}$ & $9,336 \cdot 10^{7}$ \\
\hline 25 & $4,650.10^{-7}$ & $1,204.10^{8}$ \\
\hline 26 & $2,150 \cdot 10^{-7}$ & $8,188.10^{8}$ \\
\hline & $1,000 \cdot 10^{-7}$ & \\
\hline
\end{tabular}




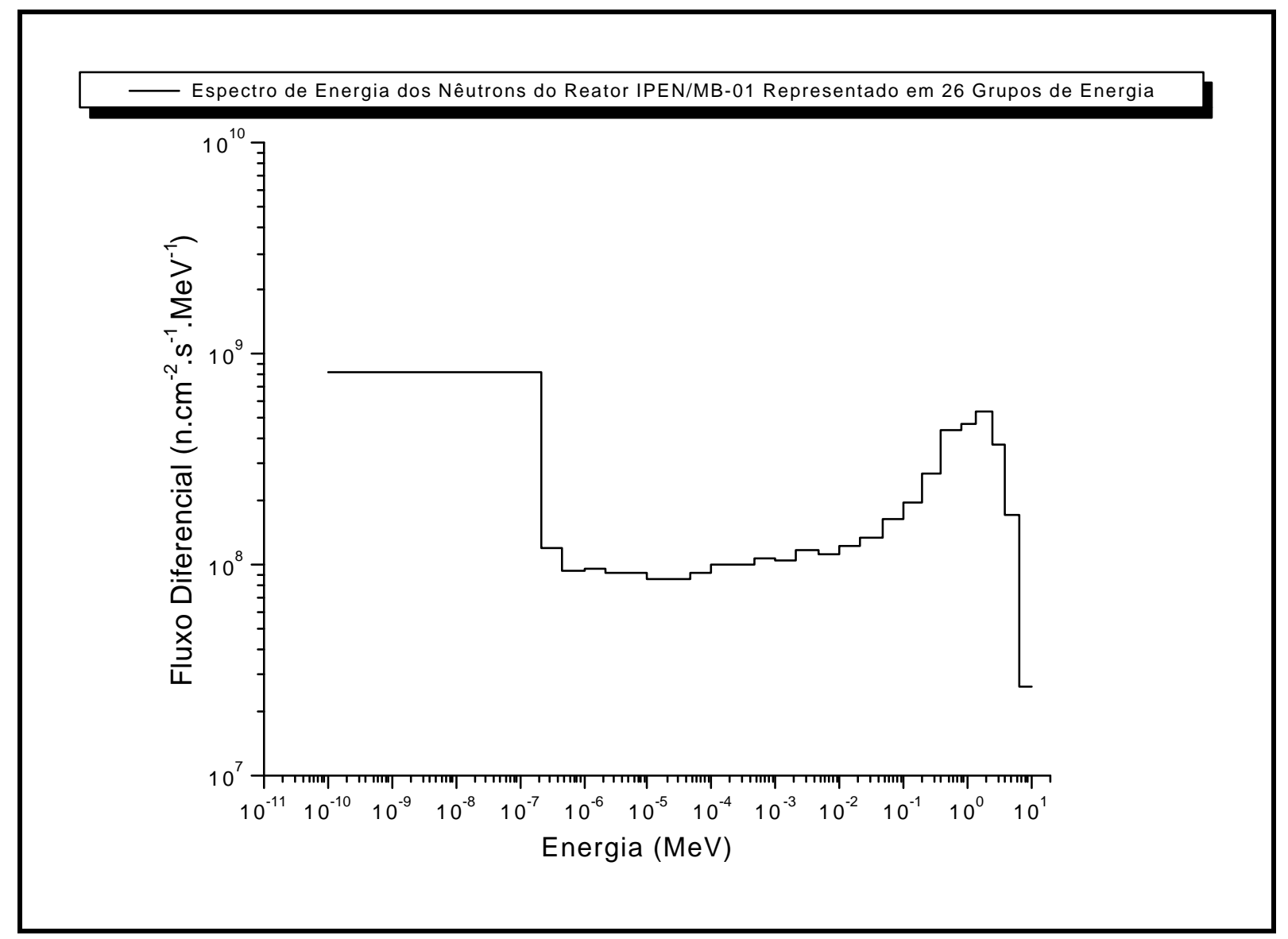

Figura 5.4 - Espectro de Energia dos Nêutrons do Reator IPEN/MB-01 em 26 Grupos de Energia ABBN GROUPS (Representação Abagyjan). 
Tabela 5.5 - Valores Absolutos de Fluxo Diferencial obtidos em 19 grupos de energia

\begin{tabular}{|c|c|c|}
\hline Grupo & $\begin{array}{c}\text { Energia } \\
(\mathrm{MeV})\end{array}$ & $\begin{array}{c}\text { Fluxo } \\
\left(\mathrm{n} / \mathrm{cm}^{2} \mathrm{sMeV}\right)\end{array}$ \\
\hline 1 & 18 & 10800 \\
\hline 2 & 16,5 & 107700 \\
\hline 3 & 14,92 & 88310 \\
\hline 4 & 14 & 96200 \\
\hline 5 & 13 & 439300 \\
\hline 6 & 12 & 782400 \\
\hline 7 & 11 & 782400 \\
\hline 8 & 10 & $3,337.10^{6}$ \\
\hline 9 & 9 & $4,647.10^{6}$ \\
\hline 10 & 8 & $9,888.10^{6}$ \\
\hline 11 & 7 & $1,996 \cdot 10^{7}$ \\
\hline 12 & 6,065 & $3,73.10^{7}$ \\
\hline 13 & 5,2 & $6,609 \cdot 10^{7}$ \\
\hline 14 & 4,4 & $1,051.10^{8}$ \\
\hline 15 & 3,68 & $1,601.10^{8}$ \\
\hline 16 & 3 & $2,716.10^{8}$ \\
\hline 17 & 2,23 & $4,651.10^{8}$ \\
\hline 18 & 1,35 & $3,99.10^{8}$ \\
\hline \multirow[t]{2}{*}{19} & 0,829 & $3,619.10^{8}$ \\
\hline & 0,5 & \\
\hline
\end{tabular}




\section{Espectro de Energia dos Nêutrons do Reator IPEN/MB-01 Representado em 19 Grupos de Energia}

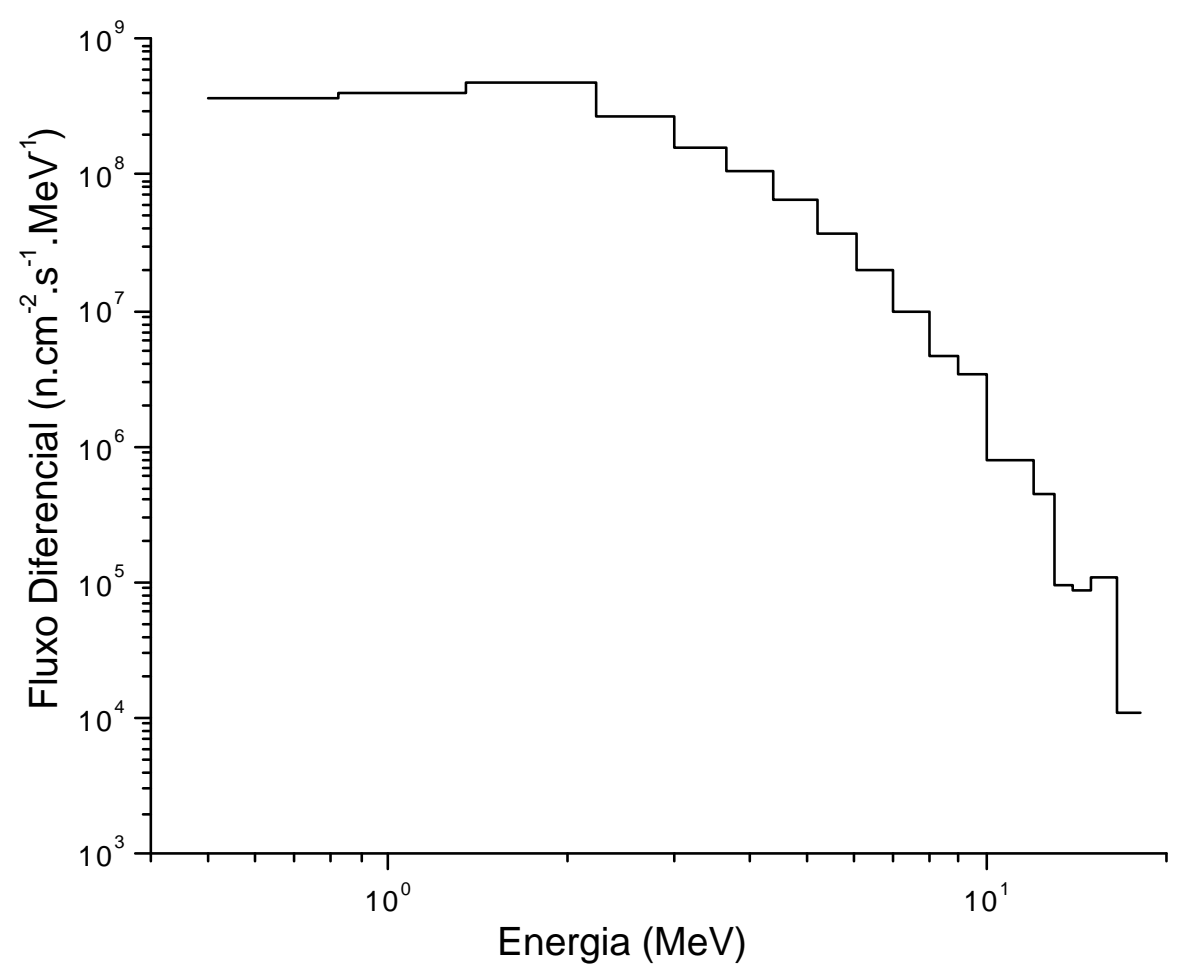

Figura 5.5 - Espectro de Energia dos Nêutrons do Reator IPEN/MB-01 em 19 Grupos de Energia (Representação SABINE-3 REMOVAL GROUPS). 


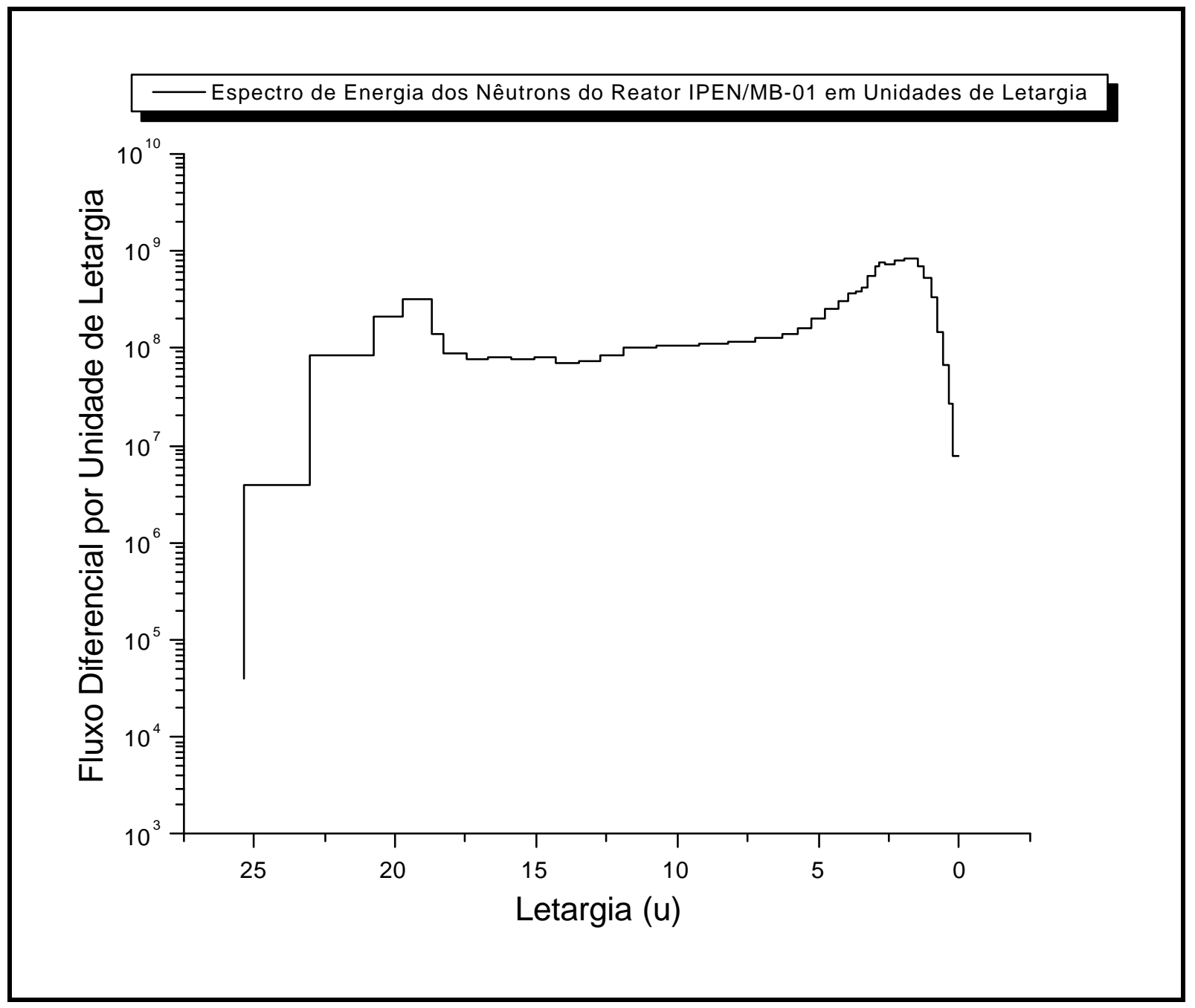

Figura 5.6 - Espectro de Energia dos Nêutrons do Reator IPEN/MB-01 em 50 Grupos em Unidades de Letargia. 
Os valores absolutos de fluxo integral de nêutrons obtidos pelo código SANDBP para o conjunto de entradas descrito no Anexo B, é dado na tabela 5.6:

Tabela 5.6 - Fluxo Integral de Nêutrons Obtido pelo Código SANDBP.

\begin{tabular}{|c|c|}
\hline Energia $(\mathrm{MeV})$ & $\begin{array}{c}\text { Fluxo Integral de } \\
\text { nêutrons }\left(\mathbf{n}_{\mathbf{c}} \mathbf{c m}^{2} \mathbf{s}\right)\end{array}$ \\
\hline$>1.10^{-10}$ & $5,1175.10^{9}$ \\
\hline$<0,20.10^{-6}$ & $8,2773.10^{8}$ \\
\hline$<0,56.10^{-6}$ & $9,7167.10^{8}$ \\
\hline$>0,1$ & $2,4757.10^{9}$ \\
\hline$>0,4$ & $2,0093.10^{9}$ \\
\hline$>0,5$ & $1,9064.10^{9}$ \\
\hline$>1,0$ & $1,3938.10^{9}$ \\
\hline
\end{tabular}

A tabela 5.7 mostra os valores absolutos de fluxo diferencial obtidos para determinados intervalos de energia:

Tabela 5.7 - Fluxo Diferencial de Nêutrons por Faixa de Energia.

\begin{tabular}{|c|c|}
\hline Faixa de Energia (MeV) & Fluxo Diferencial \\
\hline $1.10^{-10}<\Phi<0,2.10^{-6}$ & $8,0050.10^{8}$ \\
\hline $0,2.10^{-6}<\Phi<0,56.10^{-6}$ & $1,6900.10^{8}$ \\
\hline $0,56.10^{-6}<\Phi<0,1$ & $1,6723.10^{9}$ \\
\hline $0,1<\Phi<0,4$ & $4,6640.10^{8}$ \\
\hline $0,4<\Phi<0,5$ & $1,0290.10^{8}$ \\
\hline $0,5<\Phi<1,0$ & $5,1260.10^{8}$ \\
\hline $1,0<\Phi<16,0$ & $1,3938.10^{9}$ \\
\hline$\Phi>16,0$ & 0 \\
\hline
\end{tabular}




\section{COMPARAÇÃO DOS VALORES MEDIDOS E CALCULADOS}

Os valores das atividades de saturação por núcleo alvo medidas experimentalmente

e calculadas pelo código SANDBP, bem como o desvio entre os valore medidos e o calculados são apresentadas na tabela 6.1:

Tabela 6.1-Valores de $A^{\infty} / N_{a t}$ medidos e calculados, e desvio da medida para o cálculo

\begin{tabular}{|c|c|c|c|c|}
\hline \multirow[t]{2}{*}{ Reação Nuclear } & \multicolumn{2}{|c|}{$\begin{array}{l}\text { Valores das Atividades de } \\
\text { Saturação por Núcleo Alvo }\end{array}$} & \multicolumn{2}{|c|}{$\begin{array}{c}\text { Desvio dos Valores Medidos para } \\
\text { os Calculados. }\end{array}$} \\
\hline & Medidos & Calculados & $(\%)$ & (Conf) $* *$ \\
\hline $\mathrm{Mg}^{24}(\mathrm{n}, \mathrm{p}) \mathrm{Na}^{24} *$ & $2,077.10^{-18}$ & $2,287.10^{-18}$ & $-9,16$ & $-2,96$ \\
\hline $\mathrm{Mg}^{24}(\mathrm{n}, \mathrm{p}) \mathrm{Na}^{24}$ & $2,277.10^{-18}$ & $2,309 \cdot 10^{-18}$ & $-1,41$ & $-0,28$ \\
\hline $\mathrm{Al}^{27}(\mathrm{n}, \alpha) \mathrm{Na}^{24} *$ & $1,251.10^{-18}$ & $1,099.10^{-18}$ & 13,86 & 1,72 \\
\hline $\mathrm{Sc}^{45}(\mathrm{n}, \gamma) \mathrm{Sc}^{46 *}$ & $1,470.10^{-15}$ & $1,518 \cdot 10^{-15}$ & $-3,21$ & $-2,32$ \\
\hline $\mathrm{Sc}^{45}(\mathrm{n}, \gamma) \mathrm{Sc}^{46}$ & $1,944.10^{-14}$ & $2,012.10^{-14}$ & $-3,38$ & $-1,04$ \\
\hline $\mathrm{Ti}^{47}(\mathrm{n}, \mathrm{p}) \mathrm{Sc}^{47} *$ & $3,307.10^{-17}$ & $3,324 \cdot 10^{-17}$ & $-0,52$ & $-0,04$ \\
\hline $\mathrm{Ti}^{47}(\mathrm{n}, \mathrm{p}) \mathrm{Sc}^{47}$ & $3,994.10^{-17}$ & $3,360 \cdot 10^{-17}$ & 18,87 & 1,46 \\
\hline $\mathrm{Ti}^{48}(\mathrm{n}, \mathrm{p}) \mathrm{Sc}^{48 *}$ & $4,161.10^{-19}$ & $4,110.10^{-19}$ & 1,24 & 0,26 \\
\hline $\mathrm{Ni}^{58}(\mathrm{n}, \mathrm{p}) \mathrm{Co}^{58 *}$ & $1,971.10^{-16}$ & $1,939 \cdot 10^{-16}$ & 1,64 & 0,42 \\
\hline $\mathrm{Ni}^{58}(\mathrm{n}, \mathrm{p}) \mathrm{Co}^{58}$ & $2,011.10^{-16}$ & $1,959 \cdot 10^{-16}$ & 2,66 & 0,68 \\
\hline $\operatorname{In}^{115}\left(\mathrm{n}, \mathrm{n}^{\prime}\right) \operatorname{In}^{115 \mathrm{~m}} *$ & $3,570.10^{-16}$ & $3,625 \cdot 10^{-16}$ & $-1,51$ & $-0,21$ \\
\hline $\operatorname{In}^{115}\left(\mathrm{n}, \mathrm{n}^{\prime}\right) \operatorname{In}^{115 \mathrm{~m}}$ & $3,311.10^{-16}$ & $3,667 \cdot 10^{-16}$ & $-9,71$ & $-2,89$ \\
\hline $\mathrm{Au}^{197}(\mathrm{n}, \gamma) \mathrm{Au}{ }^{198 *}$ & $1,964.10^{-13}$ & $1,832.10^{-13}$ & 7,23 & 1,12 \\
\hline$A u^{197}(n, \gamma) A u^{198}$ & $2,868 \cdot 10^{-13}$ & $2,545.10^{-13}$ & 12,70 & 1,97 \\
\hline $\mathrm{U}^{238}(\mathrm{n}, \tilde{\mathrm{a}}) \mathrm{U}^{239} *$ & $2,724.10^{-14}$ & $3,041.10^{-14}$ & $-10,45$ & $-1,36$ \\
\hline$U^{238}(n, \tilde{a}) U^{239}$ & $2,670 \cdot 10^{-14}$ & $3,291.10^{-14}$ & $-18,85$ & $-2,46$ \\
\hline $\begin{array}{c}\text { Desvio Padrão } \\
\text { Total }\end{array}$ & & & 9,81 & 1,68 \\
\hline
\end{tabular}

* Folhas com cobertura de cádmio

** Razão entre o desvio entre a medida e o cálculo e o desvio padrão da medida . 
Neste capítulo são comparados ainda todos os resultados experimentais com os resultados calculados, obtidos pela Divisão de Física de Reatores IPEN/CNEN-SP através dos códigos Hammer-Technion e CITATION.

O código Hammer-Technion, utiliza a equação de transporte de nêutrons e gera as seções de choque da célula unitária, representativas do núcleo do Reator IPEN/MB-01, em dois ou quatro grupos de energia. Essas seções de choque são o resultado do colapsamento de 84 grupos de energia.

O código CITATION, que utilizando a equação de difusão de nêutrons, calcula em dois ou quatro grupos de energia a distribuição espacial do fluxo de nêutrons, a distribuição de potência, entre outros parâmetros, para o núcleo do reator.

Os valores absolutos de fluxo integral calculados pelo código CITATION em dois grupos de energia, com limiar térmico em $0,625 \mathrm{eV}$, é dado por Bitelli ${ }^{14 /}$ :

$$
\Phi_{\text {térmico }}=9,0404 \times 10^{8} \mathrm{n} / \mathrm{cm}^{2} . \mathrm{s} \quad \text { e } \quad \Phi_{\text {rápido }}=3,9664 \times 10^{9} \mathrm{n} / \mathrm{cm}^{2} . \mathrm{s}
$$

Esses valores foram calculados para a posição central do núcleo do reator, na condição de potência máxima $(100 \mathrm{~W})$ e, portanto, pode ser comparado com os valores medidos experimentalmente, onde são consideradas as mesmas condições; Uma tabela comparativa entre os fluxos obtidos através do código SANDBP e calculados pelo código CITATION é dada logo abaixo:

Tabela 6.2-Comparação dos Valores Absolutos de Fluxo Integral Medidos e Calculados

\begin{tabular}{|c|c|c|c|}
\hline Energia (MeV) & $\begin{array}{c}\text { Fluxo Integral Medido } \\
\left(\mathbf{n}^{\mathbf{c m}^{2}} \mathbf{s}\right)\end{array}$ & $\begin{array}{c}\text { Fluxo Integral Calculado } \\
\left(\mathbf{n} / \mathbf{c m}^{2} \mathbf{s}\right)\end{array}$ & $\begin{array}{c}\text { Desvio } \\
\text { Cálculo / Medida }\end{array}$ \\
\hline $\boldsymbol{\Phi}_{\text {térmico }}<\mathbf{0 , 6 2 5 . 1 0}{ }^{-6}$ & $9,7950.10^{8}$ & $9,0404.10^{8}$ & $8,3470 \%$ \\
\hline $\boldsymbol{\Phi}_{\text {rápido }}>\mathbf{0 , 6 2 5 . 1 0}^{-\mathbf{6}}$ & $4,1380.10^{9}$ & $3,9664.10^{9}$ & $4,3263 \%$ \\
\hline $\boldsymbol{\Phi}_{\text {total }}$ & $5,1175.10^{9}$ & $4,8704.10^{9}$ & $5,0735 \%$ \\
\hline
\end{tabular}




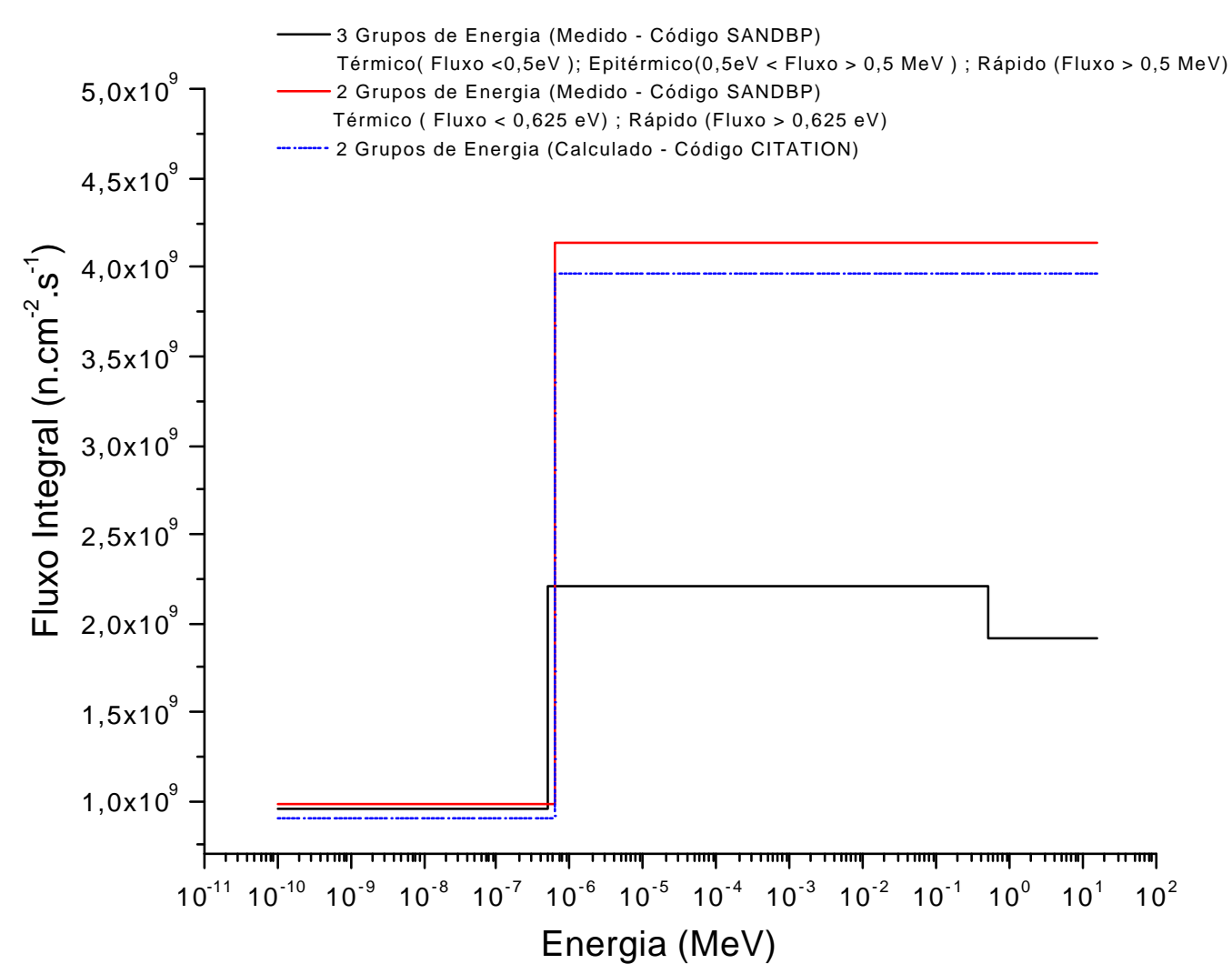

Figura 6.1 - Representação do Fluxo Integral de Nêutrons do Reator IPEN/MB-01

O Espectro de energia dos nêutrons do Reator IPEN/MB-01 medido é comparado com o espectro calculado pelo código Hammer-Technion para a célula representativa do núcleo deste reator, como pode ser visto nas figuras 6.2 e 6.3 : 


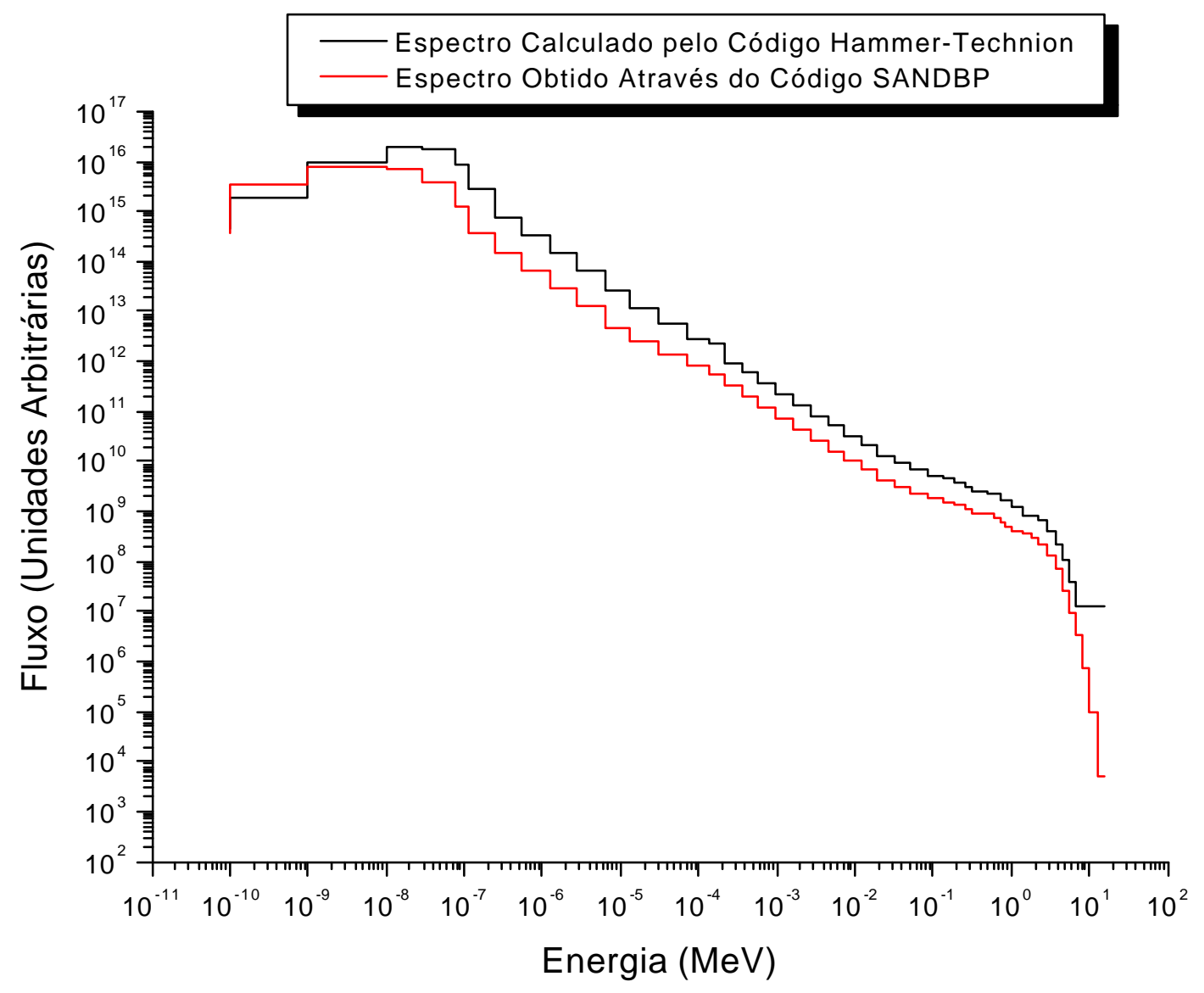

Figura 6.2 - Comparativo do Espectro Calculado pelo Hammer-Technion e Obtido Experimentalmente Através do Código SANDBP.

Na figura 6.2 o espectro que serviu de entrada para o código SANDBP (Calculado pelo Hammer-Technion) está representado em 51 pontos de energia (Primeira Aproximação do Espectro), para que possa ser melhor comparado com a estrutura energética fornecida na saída do código SANDBP.

A figura (6.3) mostra um comparativo do espectro medido e calculado em unidades de letargia: 


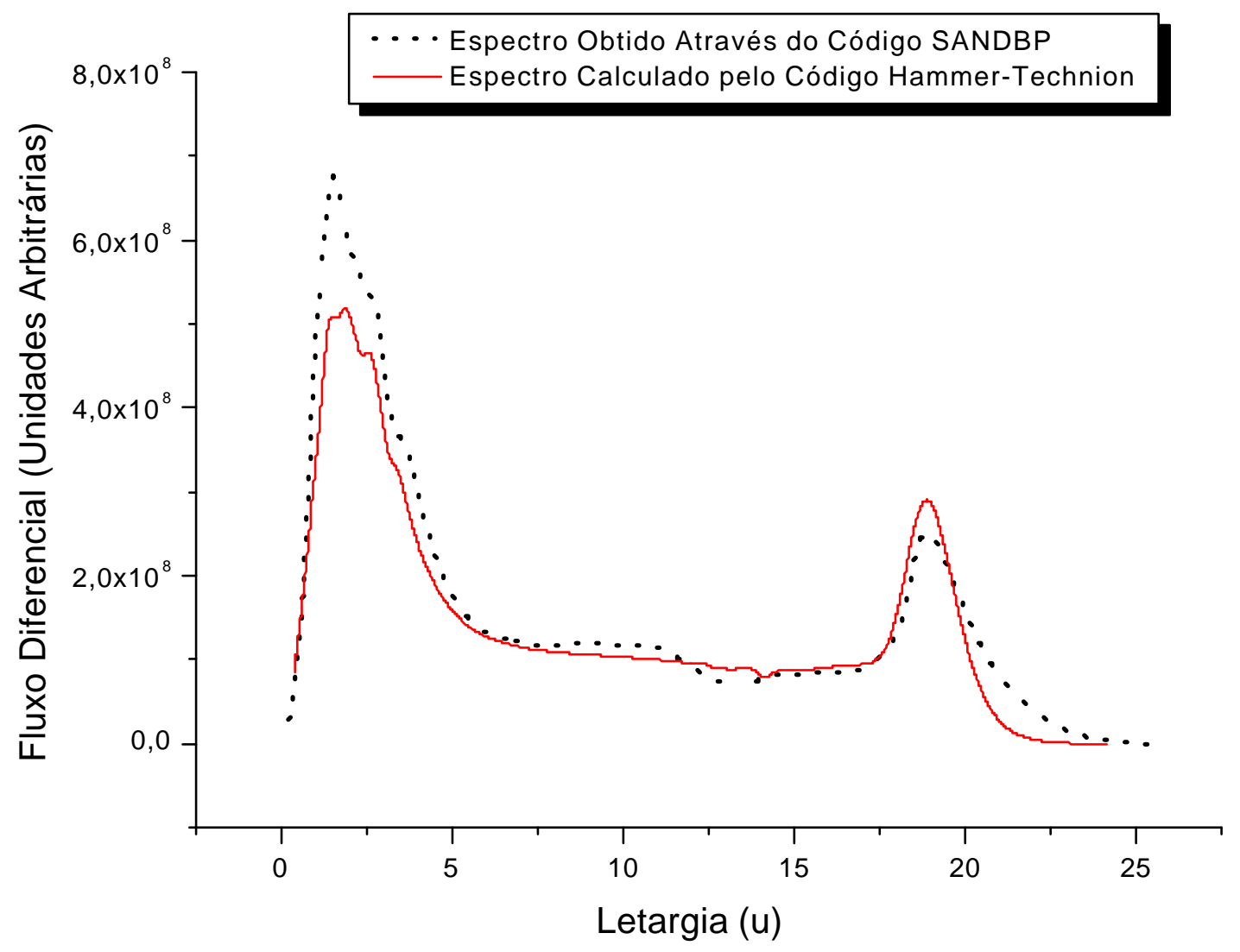

Figura 6.3 - Comparativo do Espectro Calculado pelo Hammer-Technion e Obtido Experimentalmente através do Código SANDBP em unidades de Letargia. 


\section{CONCLUSÕES}

O espectro de energia dos nêutrons obtido pela ativação de múltiplos detectores, apresentou um desvio padrão de 9,81 \% entre as atividades de saturação por núcleo alvo medidas e ajustadas a partir do espectro calculado pelo Hammer-Technion que serviu de entrada ao código SANDBP.

Observando as figuras 6.2 e 6.3, verificou-se que os valores calculados pelo código Hammer-Technion apresentam boa concordância na forma, com relação aos valores medidos pela ativação de folhas, satisfatoriamente em quase todo o espectro de energia dos nêutrons. Na região rápida do espectro o código celular Hammer-Technion parece subestimar a fuga de nêutrons do sistema.

Observando as figuras do capítulo 5 vemos que o espectro de energia dos nêutrons no núcleo do Reator IPEN/MB-01, na posição central, no moderador, é característico de um reator térmico, ou seja, pode ser dividido em três faixas de energia: a região térmica $(<0,5 \mathrm{eV})$ que pode ser aproximado por uma distribuição maxwelliana, a região intermediária $(0,5 \mathrm{eV}$ à $0,5 \mathrm{MeV})$ que tem a forma $1 / \mathrm{E}$ e a região rápida $(>0,5 \mathrm{MeV})$, que pode ser representada pelo espectro de Watt.

Os valores absolutos de fluxo integral de nêutrons do Reator IPEN/MB-01 na posição central, no moderador, foram obtidos através do código SANDBP e foram divididos nos três grupos de energia citados acima. Os resultados obtidos podem ser vistos a seguir:

$$
\begin{array}{lll}
\Phi_{\text {térmico }} & = & 9,5150.10^{8} \mathrm{n} / \mathrm{cm}^{2} . \mathrm{s} \\
\Phi_{\text {epitérmico }} & = & 2,2020.10^{9} \mathrm{n} / \mathrm{cm}^{2} . \mathrm{s} \\
\Phi_{\text {rápido }} & = & 1,9064.10^{9} \mathrm{n} / \mathrm{cm}^{2} . \mathrm{s}
\end{array}
$$




\section{$\Phi_{\text {total }} \quad=\quad 5,1175.10^{9} \mathrm{n} / \mathrm{cm}^{2} . \mathrm{S}$}

O fluxo integral foi ainda determinado em dois grupos de energia e comparado com os valores calculados pelo código CITATION, apresentando desvio de 8,3470 \% e 4,3263 $\%$ para o fluxo térmico e rápido respectivamente. $\mathrm{O}$ desvio do fluxo integral medido pelo código SANDBP e calculado pelo código CITATION em 2 grupos de energia em todo o espectro foi de $5,0735 \%$.

Através da tabela 6.2 e da figura 6.1 verifica-se ainda, conforme acima citado, boa concordância entre os valores medidos pelo código SANDBP e calculados pelo código CITATION.

\section{Proposta de Trabalhos Futuros}

Para trabalhos futuros deve-se irradiar detectores que atuem na faixa de $100 \mathrm{keV}$ à $1 \mathrm{MeV}$; Deve-se utilizar preferencial detectores na forma de ligas infinitamente diluídas, a fim de evitar os efeitos de perturbação de fluxo.

Outra proposta é calcular espectros de entrada do Reator IPEN/MB-01 usando o método de Monte Carlo e fazer uma nova comparação entre medida e cálculo.

Outro trabalho que pode ser feito é medir o espectro de energia dos nêutrons do Reator IPEN/MB-01 no combustível, o que pode ser feito utilizando a vareta experimental, que é uma vareta desmontável que permite a inserção de folhas, como as que foram utilizadas neste trabalho, dentro desta vareta combustível. Desta forma seria conhecido o comportamento energético dos nêutrons no moderador, feito neste trabalho, e no combustível. 


\section{ANEXO A - O REATOR NUCLEAR IPEN/MB-01}

\section{Introdução}

O IPEN/MB-01 é um reator nuclear genuinamente brasileiro, concebido por pesquisadores e Engenheiros do Instituto de Pesquisas Energéticas e Nucleares (IPENCNEN/SP) e da antiga COPESP (Coordenadoria para Projetos Especiais), atual CTMSP (Centro Tecnológico da Marinha em São Paulo), financiado e construído pela Marinha do Brasil, atingiu sua primeira criticalidade às 15 horas e 35 minutos do dia 9 de Novembro de 1988, sendo oficialmente entregue para operação ao IPENCNEN/SP em 28 de Novembro deste mesmo ano.

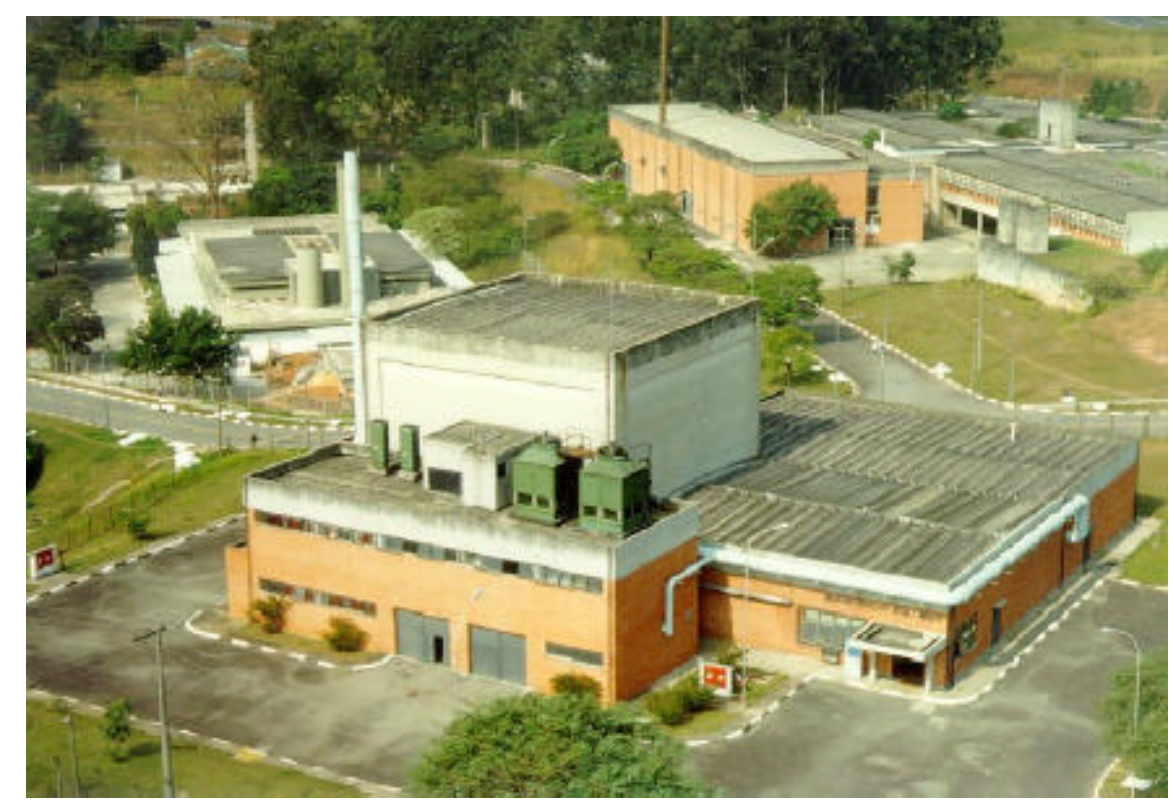

Figura A.1 Vista aérea do Reator Nuclear IPEN/MB-01.

O projeto do Reator IPEN/MB-01 foi iniciado em 1983 e suas obras foram concluídas em Julho de 1988. No mesmo mês iniciaram-se os testes dos seus vários sistemas. Cumpridas as exigências legais exigidas para o seu licenciamento, foi concedida 
em 19 de Outubro de 1988, pelas resoluções CNEN 23 e 25 a autorização para a sua operação inicial.

O Reator IPEN/MB-01 é uma instalação nuclear que permite a simulação de todas as características nucleares de um reator de grande porte em escala reduzida, sem que haja a necessidade de construir-se um complexo sistema de remoção de calor. Esse tipo de reator é conhecido mundialmente como Reator de Potência Zero ou Unidade Crítica, sendo no nosso caso, projetado para operar a uma potência máxima de 100 watts. Esses reatores

representam uma ferramenta básica, que permitem aos pesquisadores estudarem não apenas por cálculos teóricos, mas também com medidas experimentais, o desempenho e as características do núcleo de um reator de potência ou de propulsão naval, antes da sua efetiva instalação, simulando as condições de projeto na própria instalação.

A filosofia que norteou o projeto do reator IPEN/MB-01, foi no sentido de se projetar e testar um núcleo típico para uso em propulsão naval, ou seja, que o controle de reatividade se desse a partir da inserção ou retirada de barras de controle, contrariando o modelo de muitas unidades críticas em que o controle se dá pelo nível d'água no tanque moderador. Esse controle de reatividade por barras de controle é típico de reatores navais, em que se necessitam de rápidas variações de potência, a fim de se empreenderem manobras de fuga ou de perseguição.

\section{Descrição do Reator}

O primeiro núcleo do Reator IPEN/MB-01 possui a forma de paralelepípedo com dimensões ativas de 39 x 42 x 54,6 cm, sendo constituído de um arranjo de 28 x 26 varetas combustíveis e 48 tubos guias, destinados a inserção das varetas de controle/segurança, responsáveis pelo controle da reação em cadeia e desligamento do reator. Nesta configuração, dita retangular, temos um total de 680 varetas e um excesso de reatividade de aproximadamente $2415 \mathrm{pcm}$.

No entanto o núcleo do Reator Nuclear IPEN/MB-01 possibilita a montagem de diferentes arranjos críticos, ou seja, configurações de núcleos, uma vez que foi projetado para que apresentasse a versatilidade e a flexibilidade necessárias para tais finalidades. 
Para tal, a placa matriz que sustenta o núcleo do reator possui 900 furos espaçados entre si por $15 \mathrm{~mm}$, em um arranjo de 30x30. Nesta placa matriz foram montados os arranjos críticos retangulares, quadrado e cilindrizado.

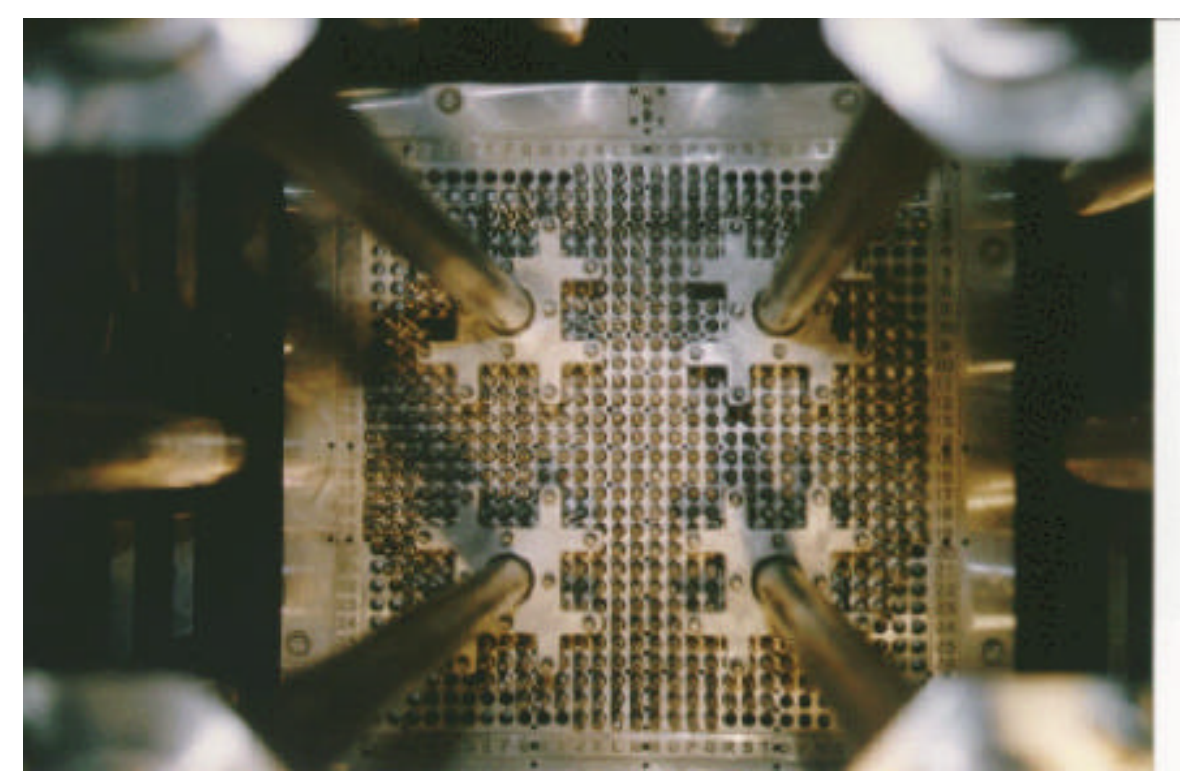

Figura A.2 Vista do Núcleo: Configuração Retangular do Reator IPEN/MB-01.

As varetas combustíveis do reator são constituídas de tubos de aço inox AISI-304, contendo em seu interior um total de 52 pastilhas combustíveis de $\mathrm{UO}_{2}$ enriquecidas a 4,3 \%. A altura ativa da coluna de pastilhas é de $54,6 \mathrm{~cm}$, sendo que cada pastilha possui uma altura de $1,05 \mathrm{~cm}$ e diâmetro de $0,849 \mathrm{~cm}$. As extremidades não ativas das varetas são preenchidas com pastilhas de $\mathrm{Al}_{2} \mathrm{O}_{3}$. Os 48 tubos guias para as varetas absorvedoras de nêutrons (barras de controle e segurança) estão dispostos em 4 grupos, contendo cada um deles 12 varetas absorvedoras, sendo dois grupos de barras de segurança e 2 grupos de controle, dispostos cada um deles em um quadrante do núcleo do reator. Cada conjunto de 12 varetas absorvedoras é unido através de um corpo central, denominado aranha, o qual é ligado a uma haste de acionamento, que por sua vez é conectada a mecanismos acionados por magnetos energizados.

Para efeito de possível modelagem em cálculos neutrônicos, apresentam-se as Tabelas A.1, A.2, A.3 e A.4 dadas a seguir, em que são apresentadas detalhadamente as composições, bem como as geometrias das varetas combustíveis e de controle do Reator IPEN/MB-01.O diagrama esquemático da vareta combustível e de controle, podem ser vistos na Figura A.3 a seguir. 

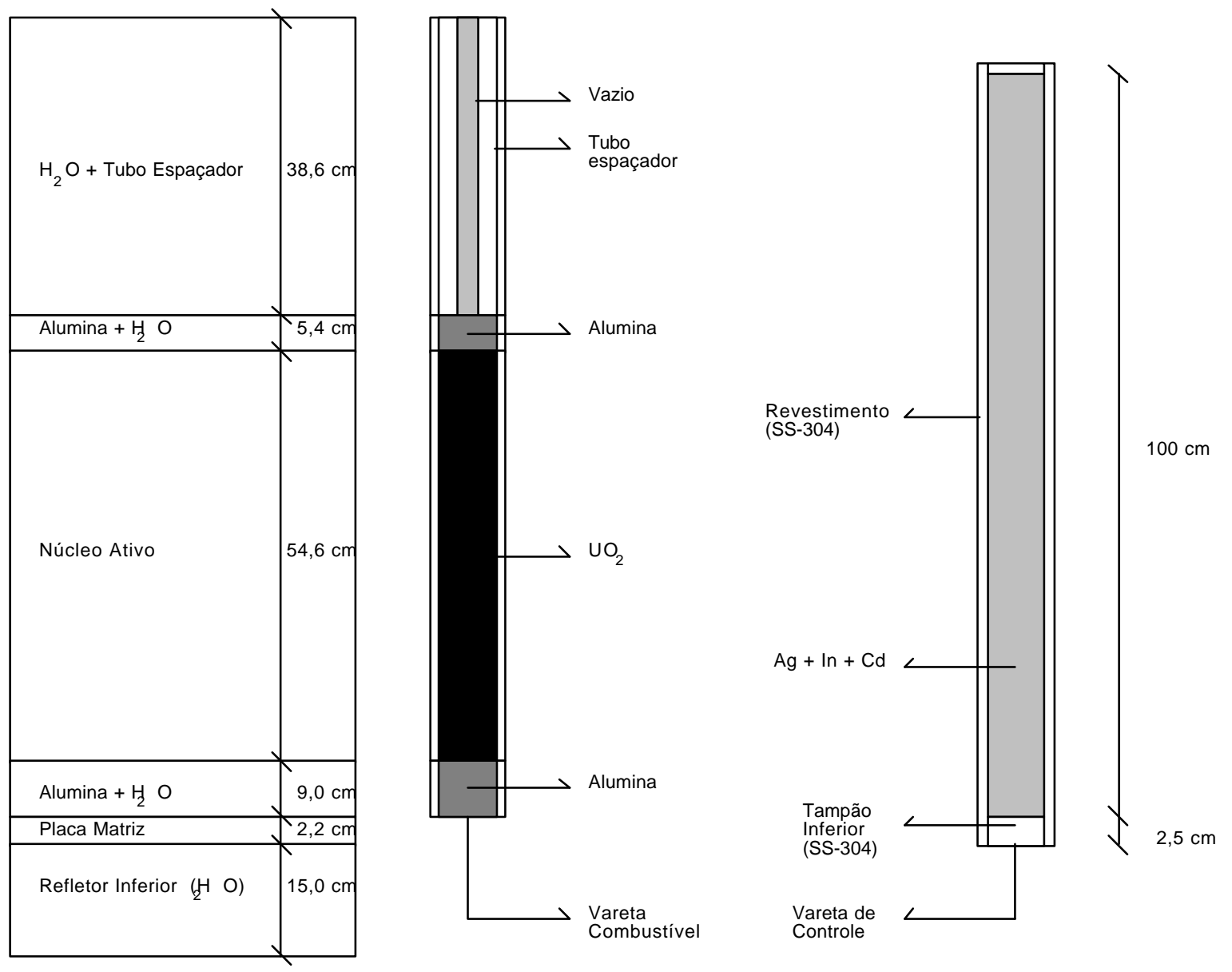

Figura A.3 Diagrama Esquemático das Varetas Combustíveis e de Controle.

A reatividade integral de cada barra de controle/segurança é suficiente para desligar o reator, ou seja, é de aproximadamente 3200 pcm. As barras de segurança apresentam as mesmas características geométricas das barras de controle, diferenciando das mesmas pelo material absorvedor de nêutrons utilizado, no caso $\mathrm{B}_{4} \mathrm{C}$ e pelo fato de que durante a operação normal do reator, serem mantidas totalmente retiradas do núcleo ativo do mesmo, com o objetivo de desligá-lo com grande margem de segurança. As barras de controle são as responsáveis, por manterem constante a população de nêutrons, quando o mesmo atinge um certo nível fixo de potência.

Quando inseridas no núcleo, as barras de controle, mantém o nível de população neutrônica, através da absorção dos mesmos em seu material estrutural composto de uma liga de Ag-In-Cd, encapsulada num revestimento de aço inox austenítico. 


\begin{tabular}{|l|l|}
\hline Material Absorvedor & Ag-In-Cd \\
\hline Diâmetro do Absorvedor & $0,832 \mathrm{~cm}$ \\
\hline Diâmetro Externo do Revestimento & $0,980 \mathrm{~cm}$ \\
\hline Espessura do Revestimento & $0,060 \mathrm{~cm}$ \\
\hline Diâmetro Externo do Tubo Guia & $1,200 \mathrm{~cm}$ \\
\hline Espessura do Tubo Guia & $0,035 \mathrm{~cm}$ \\
\hline
\end{tabular}

Tabela A.2 Dados geométricos da Vareta Combustível do Reator IPEN/MB-01.

\begin{tabular}{|c|c|}
\hline \multicolumn{2}{|l|}{ Região Ativa } \\
\hline Combustível & $\mathrm{UO}_{2}$ \\
\hline Diâmetro da Pastilha & $0,849 \mathrm{~cm}$ \\
\hline Diâmetro Externo do Revestimento & $0,980 \mathrm{~cm}$ \\
\hline Espessura do Revestimento & $0,060 \mathrm{~cm}$ \\
\hline Passo da Rede & $1,500 \mathrm{~cm}$ \\
\hline \multicolumn{2}{|l|}{ Região de Alumina } \\
\hline Diâmetro da Pastilha & $0,949 \mathrm{~cm}$ \\
\hline Diâmetro Externo do Revestimento & $0,980 \mathrm{~cm}$ \\
\hline Espessura do Revestimento & $0,060 \mathrm{~cm}$ \\
\hline \multicolumn{2}{|l|}{ Região do Tubo Espaçador } \\
\hline Diâmetro Interno & $0,730 \mathrm{~cm}$ \\
\hline Diâmetro Externo & $0,849 \mathrm{~cm}$ \\
\hline
\end{tabular}


Tabela A.3 Composição isotópica da Vareta Combustível.

\begin{tabular}{|c|c|}
\hline Pastilha Combustível & Concentração (atomos/barn-cm) \\
\hline${ }^{235} \mathrm{U}$ & $1,0034 \mathrm{E}-03$ \\
\hline${ }^{238} \mathrm{U}$ & $2,17938 \mathrm{E}-02$ \\
\hline${ }^{16} \mathrm{O}$ & $4,55138 \mathrm{E}-02$ \\
\hline Revestimento, Tubo Guia & Concentração (atomos/barn-cm) \\
\hline $\mathrm{Fe}$ & $5,67582 \mathrm{E}-02$ \\
\hline $\mathrm{Ni}$ & $8,64435 \mathrm{E}-03$ \\
\hline $\mathrm{Cr}$ & $1,72649 \mathrm{E}-02$ \\
\hline${ }^{55} \mathrm{Mn}$ & $1,59898 \mathrm{E}-03$ \\
\hline $\mathrm{Si}$ & $3,34513 \mathrm{E}-04$ \\
\hline Pastilha de Alumina & Concentração (atomos/barn-cm) \\
\hline $\mathrm{Al}$ & $4,30049 \mathrm{E}-02$ \\
\hline${ }^{16} \mathrm{O}$ & $6,45074 \mathrm{E}-02$ \\
\hline
\end{tabular}

Tabela A.4 Composição isotópica da Vareta de Controle.

\begin{tabular}{|c|c|}
\hline Absorvedor & Concentração (atomos/barn-cm) \\
\hline${ }^{107} \mathrm{Ag}$ & $2,35462 \mathrm{E}-02$ \\
\hline${ }^{109} \mathrm{Ag}$ & $2,18835 \mathrm{E}-02$ \\
\hline${ }^{113} \mathrm{In}$ & $3,42506 \mathrm{E}-04$ \\
\hline${ }^{115} \mathrm{In}$ & $7,6599 \mathrm{E}-03$ \\
\hline $\mathrm{Cd}$ & $2,72492 \mathrm{E}-03$ \\
\hline Revestimento, Tubo guia, Tampão \\
Inferior & Concentração (atomos/barn-cm) \\
\hline $\mathrm{Fe}$ & $5,67582 \mathrm{E}-02$ \\
\hline $\mathrm{Ni}$ & $8,64435 \mathrm{E}-03$ \\
\hline $\mathrm{Cr}$ & $1,72649 \mathrm{E}-02$ \\
\hline${ }^{55} \mathrm{Mn}$ & $1,59898 \mathrm{E}-03$ \\
\hline $\mathrm{Si}$ & $3,34513 \mathrm{E}-04$ \\
\hline
\end{tabular}


Todo o núcleo do reator, bem como os mecanismos de acionamento de barras, as guias para as aranhas e o amortecedor de queda de barras, é apoiado por uma estrutura suporte, fixada na parte superior por uma plataforma metálica, e na parte inferior mantida suspensa no interior do tanque moderador, o qual contém água tratada e desmineralizada, utilizada como elemento moderador da energia dos nêutrons.

Além das barras de controle e segurança, o sistema de controle de reatividade inclui um sistema de esvaziamento rápido do tanque moderador que provoca o desligamento do reator por perda do fluído moderador. No desligamento por barras, dito de primeiro nível, as 4 barras caem por gravidade no núcleo, a partir do sinal de corte de energia dos magnetos enquanto no desligamento de segundo nível, além de todas as 4 barras caírem , são abertas duas válvulas tipo borboletas de abertura rápida de $50,8 \mathrm{~cm}$ de diâmetro, situadas na parte inferior do tanque moderador, causando a retirada de toda água em aproximadamente 4 segundos. A água como sabemos, é um elemento fundamental para a moderação da energia dos nêutrons rápidos de fissão, termalizando-os e com isso tornando a probabilidade de fissão dos núcleos de Urânio-235, muito maiores para os nêutrons de baixa energia, os chamados nêutrons térmicos. A água drenada caindo por gravidade é estocada no primeiro subsolo do reator, mais propriamente no Tanque de estocagem, onde ficará armazenada até ser novamente bombeada para o tanque moderador numa futura operação do reator, ou mesmo para tratamento da mesma, através de filtragem e controle de seu nível de condutividade em um vaso trocador de leito de resina mista ou mesmo para o controle de sua temperatura em trocadores de calor aquecedores ou resfriadores.

A instrumentação nuclear utilizada no controle e segurança do reator é constituída de 10 canais nucleares, divididos por função em 2 canais de partida (detetores BF3), 2 de potência (Câmaras de Ionização Compensadas-CIC), 2 canais lineares (Câmaras de Ionização Não Compensadas-CINC), 3 canais de segurança na faixa de potência (2 CINC e 1 detetor B-10) e 1 canal de segurança na faixa de partida (Detetor BF3). Estes canais nucleares estão situados ao redor do núcleo, dentro do tanque moderador em diversas cotas axiais, posicionados dentro de tubos de alumínio estanques á água.

A instrumentação nuclear é responsável pelo processamento dos sinais gerados nos detetores nucleares. Após o processamento, estes sinais são enviados para os indicadores de potência e de período na mesa de controle do reator e para os comparadores de sinais 
que fazem a logica de proteção do reator. Existem vários valores de limiar operacionais ("set points") que constituem a rede de intertravamento dos canais nucleares. Assim, só para citar alguns exemplos, temos:

- Contagem mínima de 2 cps nos canais de partida e seu respectivo canal de segurança, para habilitar a malha de partida;

- Desligamento de sobrepotência (110 mwatts) de primeiro nível dos canais de partida;

- Desligamento por sobrepotência (120 mwatts) de segundo dos canais de partida;

- Desligamento por sobrepotência de primeiro nível (110 watts) para os canais de potência.

- Desligamento por sobrepotência de segundo nível (120 watts) para os canais de potência.

Para se atingir a contagem mínima nos canais lineares e liberar a partida do reator é necessária a utilização de uma fonte de nêutrons de Am-Be de atividade de $1 \mathrm{Ci}$ e intensidade de 2,5.10 ${ }^{6}$ nêutrons/s. Esta fonte fica armazenada no segundo subsolo do prédio do reator e durante a partida do mesmo é levada através de um pequeno carrinho preso a um cabo de aço a se posicionar na base inferior do tanque moderador, onde pode então sensibilizar os canais nucleares citados, evitando-se assim que a partida do reator se dê as cegas, ou seja numa faixa operacional em que os detetores dos canais de partida e de segurança não estejam aptos a monitorar a taxa de crescimento da população de nêutrons, quando do início de sua operação (partida do reator).

Outros intertravamentos se fazem presentes nos canais nucleares. Talvez o mais importante seja aquele referente ao período de crescimento da população neutrônica quando da supercriticalidade do reator, quando a população de nêutrons cresce exponencialmente. Assim, para períodos menores ou iguais a 17 segundos ocorrem o desligamento involuntário ("Scram") de primeiro nível, com a correspondente queda das 4 barras . Para períodos menores ou iguais a 14 segundos ocorre o "Scram" de segundo nível, ou seja a abertura das válvulas para escoamento da água do tanque moderador.

Existem vários outros intertravamentos que impedem que o reator seja ligado numa condição insegura (Intertravamentos de Segurança), a até intertravamentos que provocam o desligamento do reator (Operacionais). Podemos citar vários que vão desde a abertura da porta de acesso da Célula Crítica (Saguão onde está o núcleo do reator), até condições 
inadequadas da água moderadora, que vai desde a baixa temperatura da mesma (menor de 17 graus) até um nível inadequado de condutividade.

Todos os sistemas citados (núcleo, tanque moderador, detetores dos canais nucleares, controle das barras, etc) estão situados dentro de um prédio estanque, mantido à pressão negativa, situada na faixa de -50 a $-200 \mathrm{~Pa}$ (caso haja uma perda de estanqueidade o ar de fora é que entra), denominado de célula crítica, construído com paredes de concreto, com funções de confinamento e blindagem. Um dos intertravamentos de segurança é impedir a partida do reator caso a pressão negativa no interior da célula crítica não atinja a valores operacionais pré-estabelecidos em projeto, ou mesmo provocar o seu desligamento automático, caso a pressão negativa diminua em sua magnitude.

Dentre os inúmeros experimentos realizados nestes 18 anos de operação podemos destacar alguns deles, como teses de doutorado (medidas de índices espectrais, determinação da fração de nêutrons atrasados pela técnica de análise de ruído), mestrado ( mapeamento de fluxo com Câmaras de fissão miniatura, determinação de densidade de potência através da varredura gama de varetas combustíveis, etc), cursos de graduação fornecidos a USP (IPN0025- Física de Reatores: Experimentos no Reator Nuclear IPEN/MB-01), cursos de pós-graduação ( Medidas de Parâmetros Nucleares), cursos de formação de operadores de centrais nucleares oferecidos a Eletronuclear, experimentos de arranjos críticos considerados padrões de comparação internacional junto a OECD/NEA, experimentos de interesse a validação de metodologia de cálculo (ex: Determinação da massa crítica na curva 1/M), calibração dos canais de potência do reator através do mapeamento do fluxo de nêutrons por folhas de ativação de ouro hiper-puras, através da técnica de análise de ruído, calibração das barras de controle, coeficientes de reatividade isotérmico e de vazio, determinação do Buckling do reator, estudos da reatividade de configurações utilizando veneno queimável e este trabalho que vem se somar a tantos outros e que objetivou a determinação experimental da distribuição energética de nêutrons na região central do núcleo do reator IPEN/MB-01. 


\section{ANEXO B - CONJUNTO DE DADOS DE ENTRADA DO CÓDIGO SANDBP (INPUT).}

Neste anexo é mostrado o conjunto de dados de entrada do código SANDBP que foi utilizado para obtenção do espectro de energia dos nêutrons no Núcleo do Reator IPEN/MB-01; Durante o trabalho foram utilizados diversos conjuntos de dados de entrada.

\section{Input do Código SANDBP}

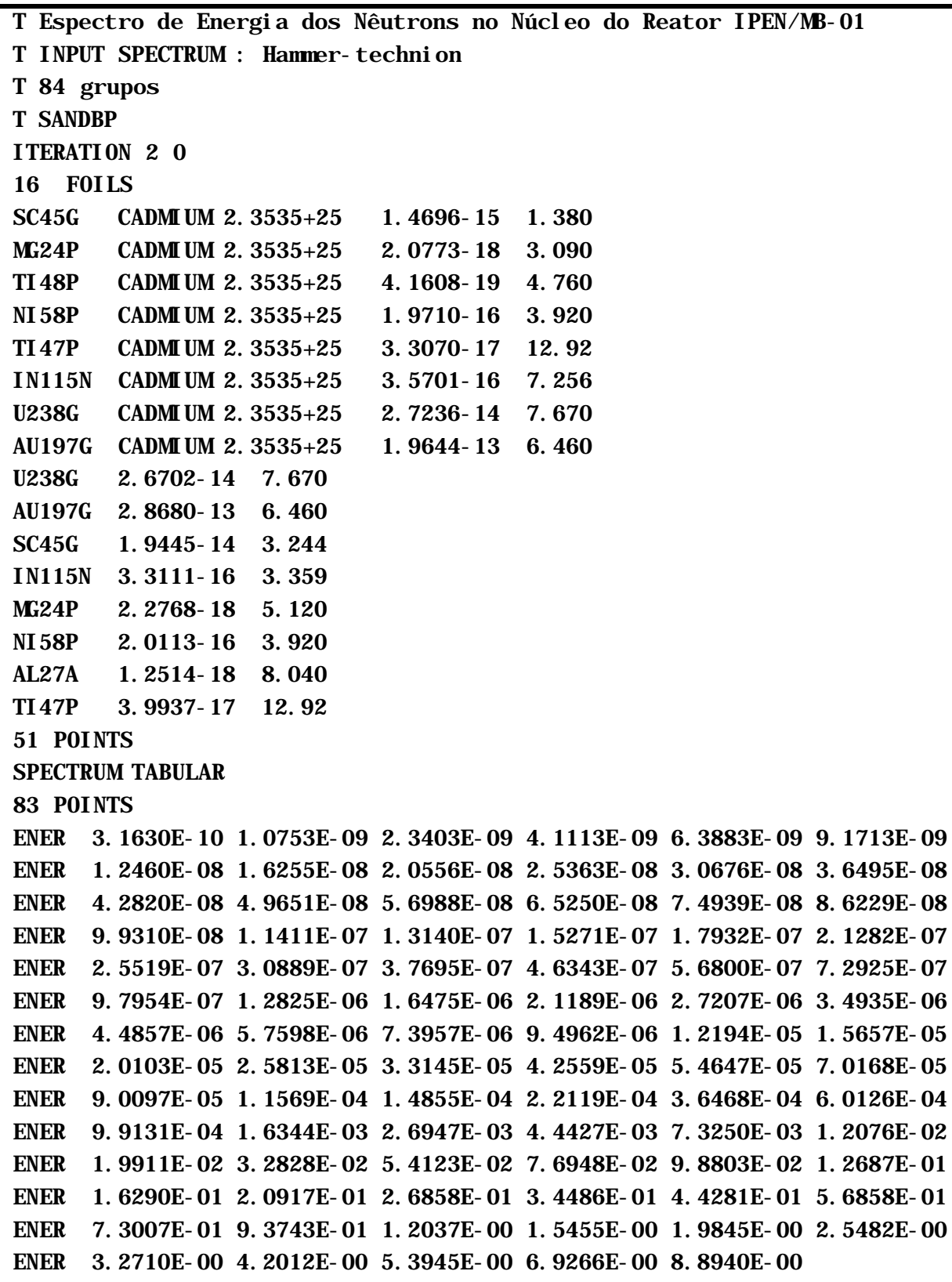


$\begin{array}{llllllll}\text { FLUX 4. 3636E+05 } & 1.9397 \mathrm{E}+06 & 4.2155 \mathrm{E}+06 & 7.0194 \mathrm{E}+06 & 1.0100 \mathrm{E}+07 & 1.3174 \mathrm{E}+07\end{array}$

$\begin{array}{llllllll}\text { FLUX } & 1.5991 \mathrm{E}+07 & 1.8325 \mathrm{E}+07 & 2.0049 \mathrm{E}+07 & 2.1071 \mathrm{E}+07 & 2.1358 \mathrm{E}+07 & 2.0986 \mathrm{E}+07\end{array}$

$\begin{array}{llllllll}\text { FLUX 2. } & 2039 \mathrm{E}+07 & 1.8635 \mathrm{E}+07 & 1.6903 \mathrm{E}+07 & 1.4881 \mathrm{E}+07 & 1.2625 \mathrm{E}+07 & 1.0305 \mathrm{E}+07\end{array}$

$\begin{array}{llllllll}\text { FLUX 8. } 0901 \mathrm{E}+06 & 6.1655 \mathrm{E}+06 & 4.5848 \mathrm{E}+06 & 3.3241 \mathrm{E}+06 & 2.3894 \mathrm{E}+06 & 1.7399 \mathrm{E}+06\end{array}$

FLUX 1. 3248E+06 $1.0379 \mathrm{E}+06 \quad 8.4207 \mathrm{E}+05 \quad 6.7637 \mathrm{E}+05 \quad 5.4621 \mathrm{E}+05 \quad 4.3254 \mathrm{E}+05$

FLUX 3. $1205 \mathrm{E}+05$ 2. $3193 \mathrm{E}+05 \quad$ 1. $8103 \mathrm{E}+05$ 1. $3883 \mathrm{E}+05$ 1. $1.0754 \mathrm{E}+05 \quad 8.2660 \mathrm{E}+04$

$\begin{array}{llllllll}\text { FLUX } & 6.4592 \mathrm{E}+04 & 4.9360 \mathrm{E}+04 & 3.3981 \mathrm{E}+04 & 3.1152 \mathrm{E}+04 & 2.4571 \mathrm{E}+04 & 1.9360 \mathrm{E}+04\end{array}$

$\begin{array}{llllllll}\text { FLUX 1. 4123E+04 } & 1.2074 \mathrm{E}+04 & 8.9860 \mathrm{E}+03 & 7.5721 \mathrm{E}+03 & 5.8878 \mathrm{E}+03 & 4.5773 \mathrm{E}+03\end{array}$

$\begin{array}{llllllll}\text { FLUX } & 3.6173 \mathrm{E}+03 & 2.8058 \mathrm{E}+03 & 2.2440 \mathrm{E}+03 & 1.5187 \mathrm{E}+03 & 9.3775 \mathrm{E}+02 & 5.7456 \mathrm{E}+02\end{array}$

$\begin{array}{lllllllll}\text { FLUX } & 3.5312 \mathrm{E}+02 & 2.1777 \mathrm{E}+02 & 1.3435 \mathrm{E}+02 & 8.2925 \mathrm{E}+01 & 5.1309 \mathrm{E}+01 & 3.2351 \mathrm{E}+01\end{array}$

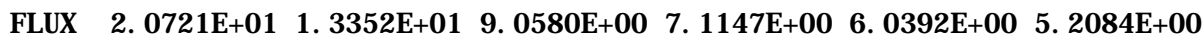

$\begin{array}{llllllll}\text { FLUX } & 4.5260 \mathrm{E}+00 & 3.9963 \mathrm{E}+00 & 3.6356 \mathrm{E}+00 & 3.2164 \mathrm{E}+00 & 2.1938 \mathrm{E}+00 & 2.5268 \mathrm{E}+00\end{array}$

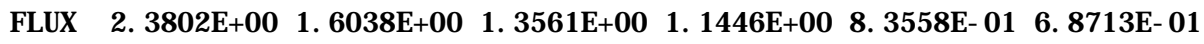

$\begin{array}{lllllll}\text { FLUX } & 3.9485 E-01 & 2.2192 E-01 & 1.0888 E-01 & 4.0965 E-02 & 1.3190 E-02\end{array}$

LI M T 3

DEV ATI ON 31

DI SCARD 10

LOW END THERMAL

HI GH END FI SSI ON

NORM 1- 10

PLOT CARDS TABLE DOSE

SMDOTH 2

MONTE- CARLO 0

NORM 1- 10

PLOT CARDS TABLE I NPUTSP

SMDOTH 2

MONTE- CARLO 0 


\section{ANEXO C - SAÍDA DO CÓDIGO SANDBP}

Neste anexo é exibida a saída do código SANDBP utilizada para o conjunto de entradas descrito no Anexo B. Para tal, foi utilizada a saída auxiliar CARDS do código, que fornece após cada iteração de ajuste uma tabela contendo os desvios entre os valores medidos e calculados de cada iteração, além da faixa energética de atuação de cada detector no espectro, e após encontrada a solução do espectro neste caso após 3 iterações, é exibido os valores absolutos das atividades de saturação por núcleo alvo e seus respectivos desvios. Está saída fornece ainda o espectro representado nas estruturas de grupo Abgajan (26 grupos) e Sabine-3 (27 e 19 grupos). Para este trabalho foram utilizadas outras saídas, como a saída DOSE que fornece os valores absolutos de fluxo integral acima e abaixo de determinadas energias, e as saídas PLOT, INPUTSP, INTSPEC, DFDE, que são saídas gráficas e não são mostradas neste anexo.

\section{Saída do Código SANDBP}

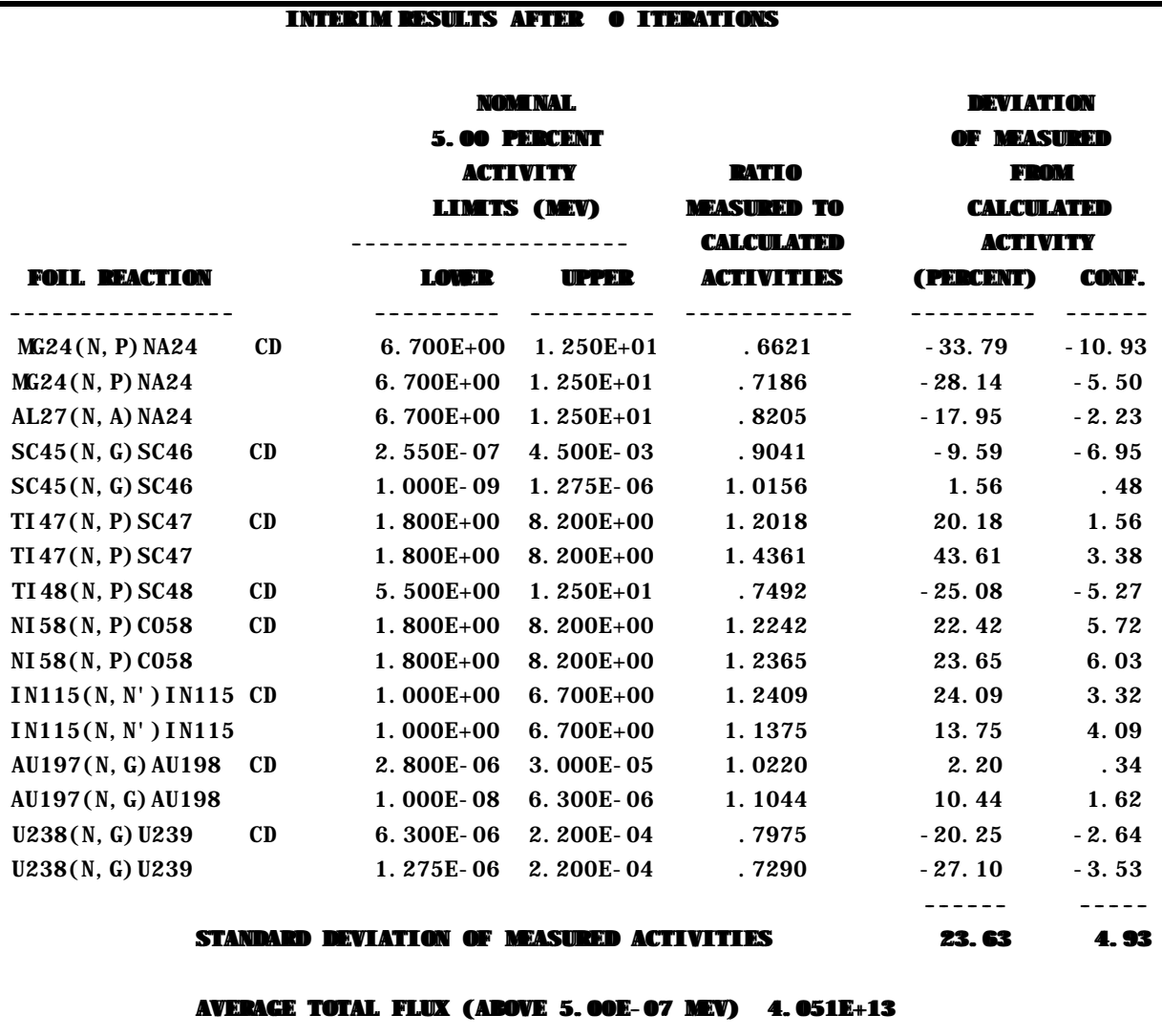




\begin{tabular}{|c|c|c|c|c|c|c|}
\hline \multirow[b]{2}{*}{ FOI L REACTI ON } & & \multicolumn{2}{|c|}{$\begin{array}{l}\text { WOMHI NAL } \\
\text { 5. OO PERCE WT } \\
\text { ACTI VI TY } \\
\text { LI MUIS US U MEV\| }\end{array}$} & \multicolumn{3}{|c|}{$\begin{array}{l}\text { DEVI ATI ON } \\
\text { OF MEASURED } \\
\text { FROM } \\
\text { CALCELATED } \\
\text { ACTI VITY }\end{array}$} \\
\hline & & LOWE R & UPPE R & ACTI VIITIES & 《 PE RCE MT ) & COMf. \\
\hline (n) & & 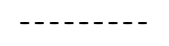 & (n. & . & (n. & - . \\
\hline MG24( N, P) NA24 & $\mathrm{CD}$ & 6. $700 \mathrm{E}+00$ & 1. $250 \mathrm{E}+01$ & 8314 & - 16. 86 & -5.46 \\
\hline MG24( N, P) NA24 & & 6. $700 \mathrm{E}+00$ & 1. $250 \mathrm{E}+01$ & .9022 & -9.78 & -1.91 \\
\hline AL27( N, A) NA24 & & 6. $700 E+00$ & 1. $250 \mathrm{E}+01$ & 1. 0388 & 3. 88 & .48 \\
\hline SC45( N, G) SC46 & $\mathrm{CD}$ & 2. $550 \mathrm{E}-07$ & 4. $500 \mathrm{E}-03$ & .9574 & -4.26 & -3.08 \\
\hline $\mathrm{SC} 45(\mathrm{~N}, \mathrm{G}) \mathrm{SC} 46$ & & 1. $000 \mathrm{E}-09$ & 1. $275 \mathrm{E}-06$ & .9940 & -.60 & -.18 \\
\hline TI 47( N, P) SC47 & $\mathrm{CD}$ & 1. $800 E+00$ & 8. $200 E+00$ & 1. 0559 & 5.59 & .43 \\
\hline TI 47( N, P) SC47 & & 1. $800 E+00$ & 8. $200 \mathrm{E}+00$ & 1. 2617 & 26. 17 & 2.03 \\
\hline TI 48( N, P) SC48 & $\mathrm{CD}$ & 5. $500 E+00$ & 1. $250 \mathrm{E}+01$ & .9323 & -6.77 & -1.42 \\
\hline NI 58( N, P) CO58 & $\mathrm{CD}$ & 1. $800 \mathrm{E}+00$ & 8. $200 E+00$ & 1. 0830 & 8. 30 & 2. 12 \\
\hline $\mathrm{Nl} 58(\mathrm{~N}, \mathrm{P}) \mathrm{CO58}$ & & 1. $800 E+00$ & 8. $200 E+00$ & 1. 0938 & 9. 38 & 2. 39 \\
\hline I N115( N, N') । N115 & $C D$ & 1. $000 E+00$ & 5. $500 \mathrm{E}+00$ & 1. 0384 & 3. 84 & .53 \\
\hline I N115( N, N') I N115 & & 1. $000 E+00$ & 5. $500 \mathrm{E}+00$ & .9518 & -4.82 & -1.43 \\
\hline AU197( N, G) AU198 & $C D$ & 2. $800 \mathrm{E}-06$ & 3. $000 \mathrm{E}-05$ & 1. 0761 & 7. 61 & 1. 18 \\
\hline AU197( N, G) AU198 & & 1. $000 \mathrm{E}-08$ & 6. $300 \mathrm{E}-06$ & 1. 1394 & 13. 94 & 2. 16 \\
\hline U238( N, G) U239 & $\mathrm{CD}$ & 6. $300 \mathrm{E}-06$ & 2. $200 \mathrm{E}-04$ & .8609 & -13.91 & -1.81 \\
\hline U238( N, G) U239 & & 2. $550 \mathrm{E}-07$ & 2. $200 \mathrm{E}-04$ & .7830 & -21.70 & -2.83 \\
\hline $\begin{array}{r}\text { STA } \\
\text { AVER }\end{array}$ & $\begin{array}{l}\text { MDARD } \\
\text { IGE T }\end{array}$ & $\begin{array}{l}\text { II ATI ONI OF M } \\
\text { FLUX | ABOVE }\end{array}$ & $\begin{array}{l}\text { EASURED ACT } \\
5.00 E-07\end{array}$ & $\begin{array}{l}\text { I V\| TI ES } \\
\text { AEV) } \quad 4.180 E+13\end{array}$ & 12.35 & 2.30 \\
\hline
\end{tabular}

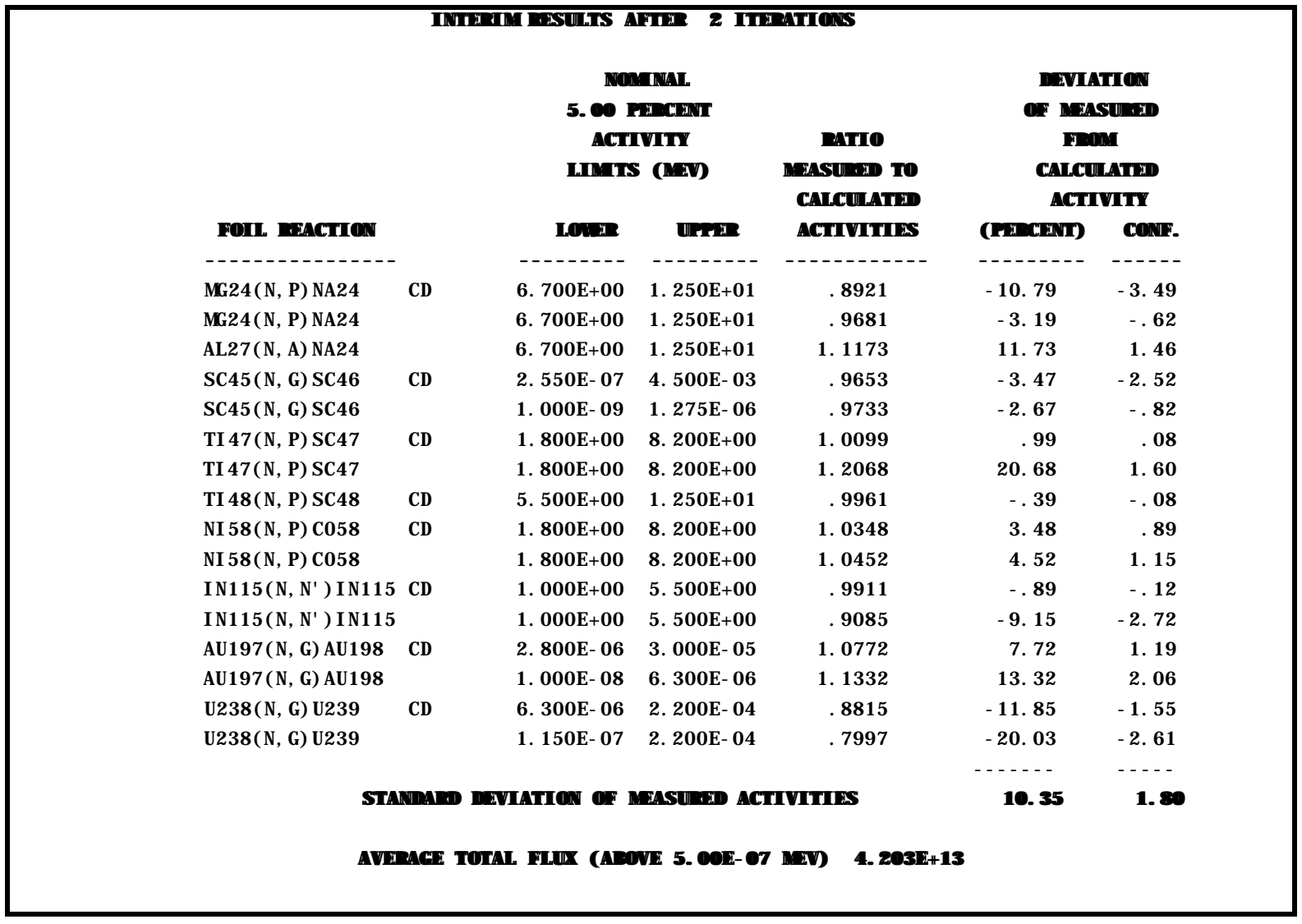




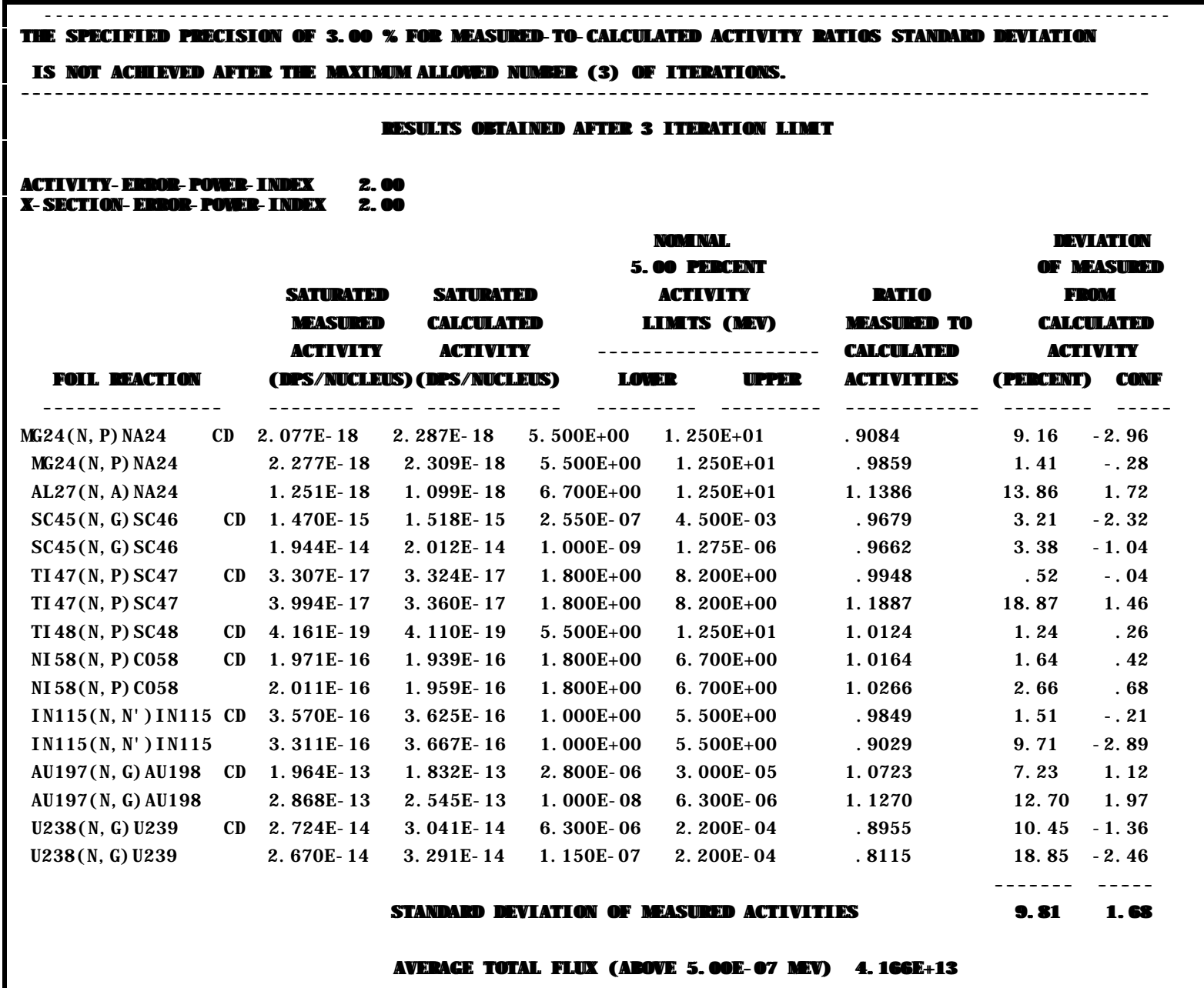

A seguir são exibidas tabelas que permitem avaliar o efeito das incertezas das atividades de saturação por núcleo alvo e das seções de choque, que modificam o espectro através da função peso do termo de correção da equação (4.1). Essas incertezas são introduzidas no input do código na linha ITERATION 1 n, onde 1 refere-se a incerteza da atividade de saturação por núcleo alvo e $n$ da seção de choque. Podem assumir os valores 0,1 e 2, no qual 0 indica que não é introduzido peso nas incertezas. 
Tabela C.1 - Efeito da Função Peso nos Valores de Atividade e Seção de Choque na Modificação do Espectro - Dados de Entrada (Input)

\begin{tabular}{|c|c|c|c|}
\hline \multirow{2}{*}{$\begin{array}{c}\text { Reação } \\
\text { Nuclear }\end{array}$} & \multirow{2}{*}{$\begin{array}{c}\text { 90\% Faixa de Resposta do } \\
\text { Detector } \\
(\mathrm{MeV})\end{array}$} & \multicolumn{2}{|c|}{$\begin{array}{c}\text { Desvio do Valor Medido e } \\
\text { Calculado }\end{array}$} \\
\hline & & $\%$ & Conf \\
\hline $\mathrm{Mg} 24^{*}$ & $6,7 \cdot 10^{0}{ }^{\prime} \quad 1,3 \cdot 10^{+1}$ & $-9,16$ & $-2,96$ \\
\hline $\mathrm{Mg} 24 \mathrm{P}$ & $6,7.10^{0}$ & $-1,41$ & $-0,28$ \\
\hline Al27A* & $6,7 \cdot 10^{0}$ & 13,86 & 1,72 \\
\hline Sc45G* & $-4,5.10^{-3}$ & $-3,21$ & $-2,32$ \\
\hline Sc45G & $1,0.10^{-9} \ldots 1,3 \cdot 10^{-6}$ & $-3,38$ & $-1,04$ \\
\hline Ti47P* & $1,8.10^{0}$ & $-0,52$ & $-0,04$ \\
\hline Ti47P & $1,8.10^{\circ} \_8,2.10^{0}$ & 18,87 & 1,46 \\
\hline Ti48P* & $5,5.10^{0}$ & 1,24 & 0,26 \\
\hline Ni58P* & $8,2.10^{0}$ & 1,64 & 0,42 \\
\hline Ni58P & $8,2.10^{0}$ & 2,66 & 0,68 \\
\hline In $115 \mathrm{~N}$ & $6,7.10^{+1}$ & $-1,51$ & $-0,21$ \\
\hline In115N & $1,0.10^{0}$ & $-9,71$ & $-2,89$ \\
\hline Au197G* & $2,8.10^{-6}$ & 7,23 & 1,12 \\
\hline Au197G & $6,3 \cdot 10^{-6}$ & 12,70 & 1,97 \\
\hline
\end{tabular}




\begin{tabular}{|c|cc|c|c|}
\hline Th232G* & $1,4 \cdot 10^{-5}$ & $4,5 \cdot 10^{-3}$ & $-10,45$ & $-1,36$ \\
\hline U238G & $1,3 \cdot 10^{-6}$ & $2,2 \cdot 10^{-4}$ & $-18,85$ & $-2,46$ \\
\hline
\end{tabular}

Tabela C.2 - Efeito da Função Peso nos Valores de Atividade e Seção de Choque, na Modificação do Espectro - Após 3 iterações

\begin{tabular}{|c|c|c|c|c|c|c|c|c|}
\hline \multirow{3}{*}{$\begin{array}{l}\text { Reação } \\
\text { Nuclear }\end{array}$} & \multicolumn{8}{|c|}{ Desvio dos Valores Medidos e Calculados } \\
\hline & \multicolumn{2}{|c|}{$\operatorname{Peso}(0,0)$} & \multicolumn{2}{|c|}{$\operatorname{Peso}(2,0)$} & \multicolumn{2}{|c|}{$\operatorname{Peso}(0,2)$} & \multicolumn{2}{|c|}{$\operatorname{Peso}(2,2)$} \\
\hline & $\%$ & Conf & $\%$ & Conf & $\%$ & Conf & $\%$ & Conf \\
\hline $\operatorname{Mg} 24 *$ & $-11,31$ & $-3,66$ & $-9,16$ & $-2,96$ & $-11,58$ & $-3,75$ & $-8,08$ & $-2,62$ \\
\hline $\mathrm{Mg} 24 \mathrm{P}$ & $-3,75$ & $-0,73$ & $-1,41$ & $-0,28$ & $-4,05$ & $-0,78$ & $-0,25$ & $-0,05$ \\
\hline Al27A* & 11,27 & 1,40 & 13,86 & 1,72 & 10,52 & 1,31 & 15,49 & 1,43 \\
\hline Sc45G* & $-3,70$ & $-2,68$ & $-3,21$ & $-2,32$ & $-10,02$ & $-7,26$ & $-11,66$ & $-8,45$ \\
\hline $\mathrm{Sc} 45 \mathrm{G}$ & $-2,42$ & $-0,75$ & $-3,38$ & $-1,04$ & $-12,04$ & $-3,71$ & $-13,29$ & $-4,10$ \\
\hline Ti47P* & $-1,41$ & $-0,11$ & $-0,52$ & $-0,04$ & 3,00 & 0,23 & 2,66 & 0,21 \\
\hline Ti47P & 17,81 & 1,38 & 18,87 & 1,46 & 23,07 & 1,79 & 22,68 & 1,76 \\
\hline Ti48P* & $-1,12$ & $-0,24$ & 1,24 & 0,26 & $-1,15$ & $-0,24$ & 2,44 & 0,51 \\
\hline Ni58P* & 0,89 & 0,23 & 1,64 & 0,42 & 5,29 & 1,35 & 4,47 & 1,14 \\
\hline Ni58P & 1,90 & 0,49 & 2,66 & 0,68 & 6,34 & 1,62 & 5,51 & 1,41 \\
\hline In115N* & $-2,82$ & $-0,39$ & $-1,51$ & $-0,21$ & 2,15 & 0,30 & 2,81 & 0,39 \\
\hline In115N & $-10,92$ & $-3,25$ & $-9,71$ & $-2,89$ & $-6,36$ & $-1,89$ & $-5,75$ & $-1,71$ \\
\hline $\mathrm{Au} 197 \mathrm{G}^{*}$ & 4,69 & 0,73 & 7,23 & 1,12 & 1,65 & 0,26 & $-1,26$ & $-0,20$ \\
\hline $\mathrm{Au} 197 \mathrm{G}$ & 11,07 & 1,71 & 12,70 & 1,97 & 5,59 & 0,86 & 2,99 & 0,46 \\
\hline $\mathrm{U} 238 \mathrm{G} *$ & $-0,18$ & $-0,02$ & $-10,45$ & $-1,36$ & $-1,05$ & $-0,14$ & $-4,47$ & $-0,58$ \\
\hline U238G & $-10,01$ & $-1,30$ & $-18,85$ & $-2,46$ & $-11,35$ & $-1,48$ & $-14,29$ & $-1,86$ \\
\hline Desvio & 8,11 & 1,67 & $\mathbf{9 , 8 1}$ & 1,68 & 9,40 & 2,55 & 9,90 & 2,74 \\
\hline
\end{tabular}


Tabela C.3 - Resultados das Modificações do Espectro Usando Diferentes Fatores de Peso para a Atividade e Seção de Choque.

\begin{tabular}{|c|c|c|c|c|c|c|c|c|}
\hline \multirow{3}{*}{ Fator Peso } & \multicolumn{8}{|c|}{ Desvio Médio dos Valores de Atividade Medidos e Calculados Após: } \\
\hline & \multicolumn{2}{|c|}{ O Iterações } & \multicolumn{2}{|c|}{1 Iterações } & \multicolumn{2}{|c|}{2 Iterações } & \multicolumn{2}{|c|}{3 Iterações } \\
\hline & $\%$ & Conf & $\%$ & Conf & $\%$ & Conf & $\%$ & Conf \\
\hline$(0,0)$ & 23,63 & 4,93 & 11,60 & 2,34 & 8,85 & 1,80 & 8,11 & 1,67 \\
\hline$(2,0)$ & 23,63 & 4,93 & 12,35 & 2,30 & 10,35 & 1,80 & 9,81 & 1,68 \\
\hline$(0,2)$ & 23,63 & 4,93 & 12,39 & 2,82 & 9,95 & 2,51 & 9,40 & 2,55 \\
\hline$(2,2)$ & 23,63 & 4,93 & 12,89 & 3,03 & 10,55 & 2,74 & 9,90 & 2,74 \\
\hline
\end{tabular}

\section{ANEXO D - FLUXO DE NÊUTRONS DO REATOR IPEN/MB-01 E SUAS INCERTEZAS}

Neste anexo será exibido o espectro de energia dos nêutrons obtido através da análise das incertezas pelo método de Monte Carlo, contida no código SANDBP.

Os valores absolutos das atividades de saturação por núcleo alvo calculadas pelo código SANDBP e os valores absolutos de fluxo integral, bem como o desvio padrão destas medidas são apresentados nas tabelas abaixo:

Tabela D.1 - Valores Absolutos de das Atividades de Saturação por Núcleo Alvo Calculadas e Suas Incertezas

\begin{tabular}{|c|c|c|}
\hline Reação & $\begin{array}{c}\text { Atividade Calculada } \\
\text { (Bq/ Núcleo Alvo })\end{array}$ & $\begin{array}{c}\text { Desvio Padrão da } \\
\text { Atividade Calculada } \\
\text { (Bq/Núcleo Alvo })\end{array}$ \\
\hline $\mathrm{Mg} 24^{*}$ & $2,272.10^{-18}$ & $3,351.10^{-20}$ \\
\hline $\mathrm{Mg} 24 \mathrm{P}$ & $2,299.10^{-18}$ & $4,282.10^{-20}$ \\
\hline $\mathrm{Al} 27 \mathrm{~A}^{*}$ & $1,094.10^{-18}$ & $1,804.10^{-20}$ \\
\hline $\mathrm{Sc}^{-15} \mathrm{G}^{*}$ & $1,506.10^{-15}$ & $2,652.10^{-17}$ \\
\hline $\mathrm{Sc} 45 \mathrm{G}$ & $2,007.10^{-14}$ & $4,695.10^{-16}$ \\
\hline $\mathrm{Ti} 47 \mathrm{P}^{*}$ & $3,305.10^{-17}$ & $9,718.10^{-19}$ \\
\hline
\end{tabular}




\begin{tabular}{|c|c|c|}
\hline Ti47P & $3,369 \cdot 10^{-17}$ & $1,086 \cdot 10^{-18}$ \\
\hline Ti48P* & $4,100.10^{-19}$ & $1,012.10^{-20}$ \\
\hline Ni58P* & $1,933.10^{-16}$ & $4,171 \cdot 10^{-18}$ \\
\hline Ni58P & $1,948.10^{-16}$ & $5,220.10^{-18}$ \\
\hline In115N* & $3,620.10^{-16}$ & $9.570 .10^{-18}$ \\
\hline In115N & $3,642.10^{-16}$ & $8.033 .10^{-18}$ \\
\hline Au197G* & $1,853.10^{-13}$ & $1.421 .10^{-14}$ \\
\hline $\mathrm{Au} 197 \mathrm{G}$ & $2,573.10^{-13}$ & $1.259 .10^{-14}$ \\
\hline $\mathrm{U} 238 \mathrm{G} *$ & $3,020.10^{-14}$ & $1.712 .10^{-15}$ \\
\hline U238G & $3,274.10^{-14}$ & $1.753 .10^{-15}$ \\
\hline
\end{tabular}

* Folhas com cobertura de cádmio

Tabela D.2 - Valores Absolutos de Fluxo Integral e suas Respectivas Incertezas

\begin{tabular}{|c|c|c|}
\hline Energia (MeV) & $\begin{array}{c}\text { Fluxo Integral de } \\
\text { nêutrons }\left(\mathbf{n}^{\text {(cm }} \mathbf{~}^{\mathbf{s}} \text { ) }\right.\end{array}$ & $\begin{array}{c}\text { Desvio Padrão do Fluxo } \\
\text { Integral }\end{array}$ \\
\hline$>1.10^{-10}$ & $5,1312.10^{9}$ & $1,6092.10^{8}$ \\
\hline$<0,20.10^{-6}$ & $8,3502.10^{8}$ & $4,9141.10^{7}$ \\
\hline$<0,56.10^{-6}$ & $9,8087.10^{8}$ & $4,9744.10^{7}$ \\
\hline$>0,1$ & $2,4771.10^{9}$ & $6,7100.10^{7}$ \\
\hline$>0,4$ & $2,0110.10^{9}$ & $6,1730.10^{7}$ \\
\hline$>0,5$ & $1,9040.10^{9}$ & $6,3896.10^{7}$ \\
\hline$>1,0$ & $1,3899.10^{9}$ & $4,4776.10^{7}$ \\
\hline
\end{tabular}

O Gráfico abaixo mostra o espectro de energia dos nêutrons no núcleo do Reator IPEN/MB-01 em 51 pontos de energia obtido através do código SANDBP, utilizando a análise de incertezas de Monte Carlo. 
Variação Energética do Fluxo de Nêutrons do Reator IPEN/MB-01 e suas Incertezas em 51 Pontos de Energia

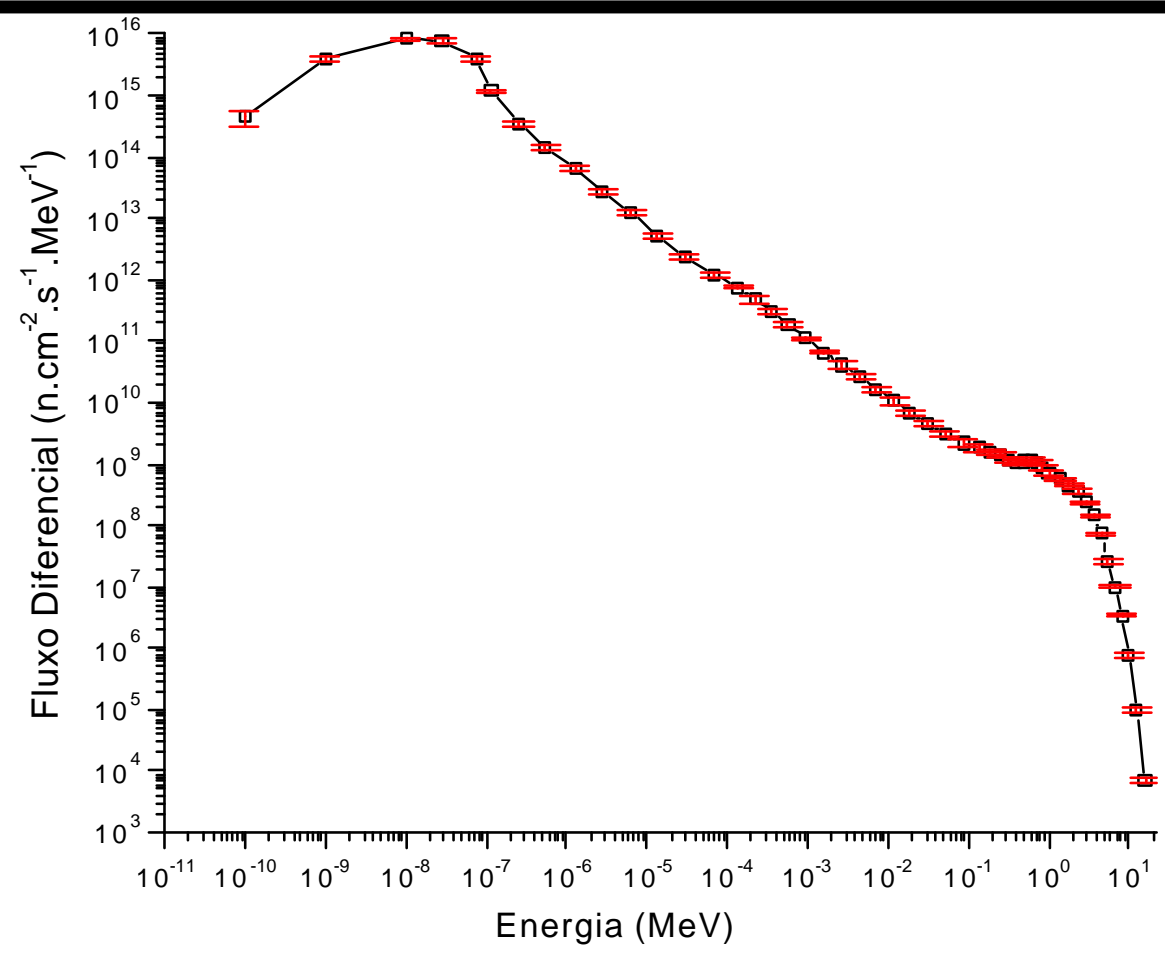

Figura D.1 - Variação Energética do Fluxo Diferencial de Nêutrons em 51 Pontos de Energia.

\section{ANEXO E - CURVAS DE SEÇÃO DE CHOQUE DOS MATERIAIS UTILIZADOS COMO DETECTORES DE ATIVAÇÃO}

Neste anexo serão exibidas as curvas de seção de choque dos materiais utilizados como detectores de ativação. 

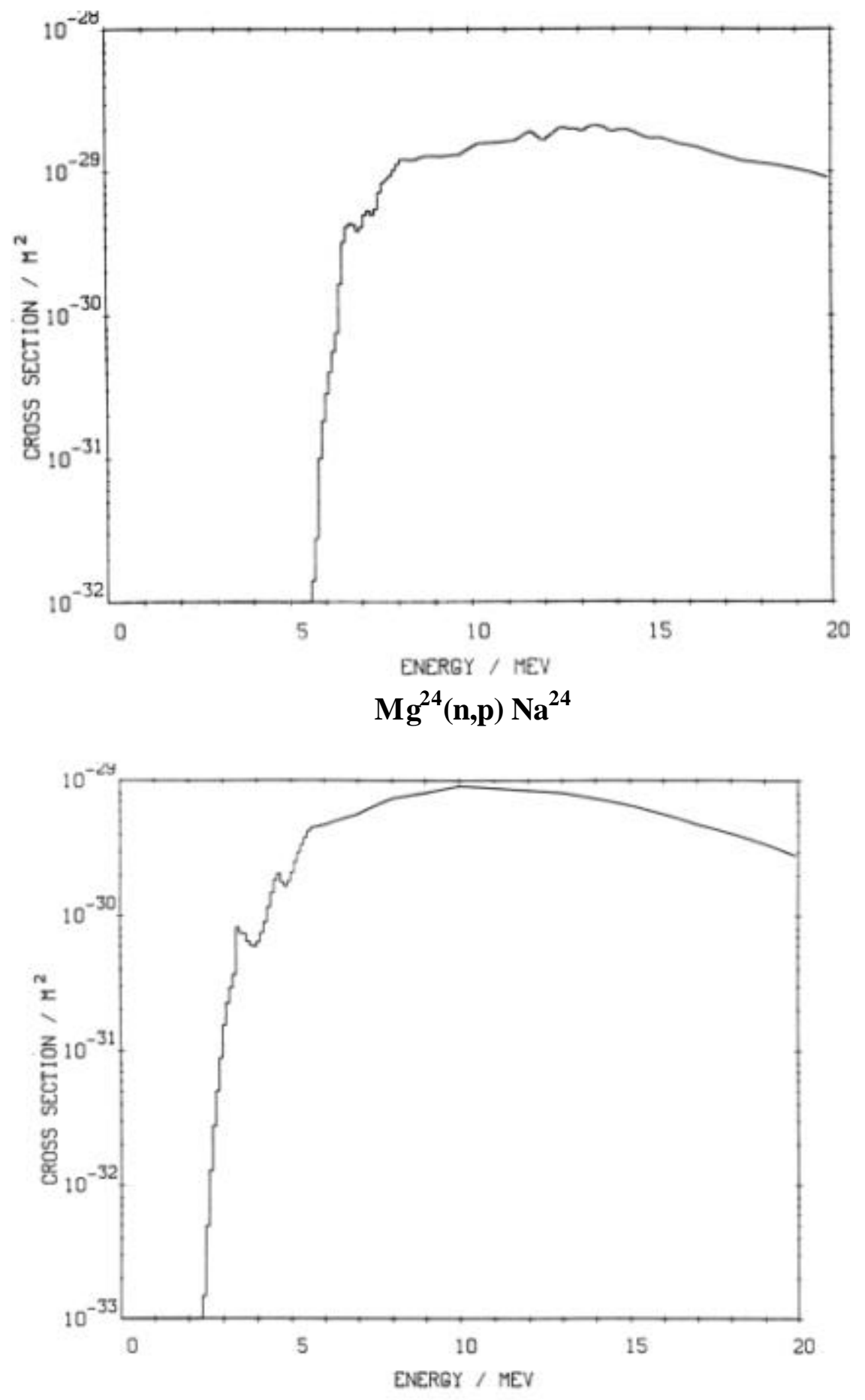

$\mathrm{Al}^{27}(\mathrm{n}, \boldsymbol{\alpha}) \mathrm{Na}^{24}$ 


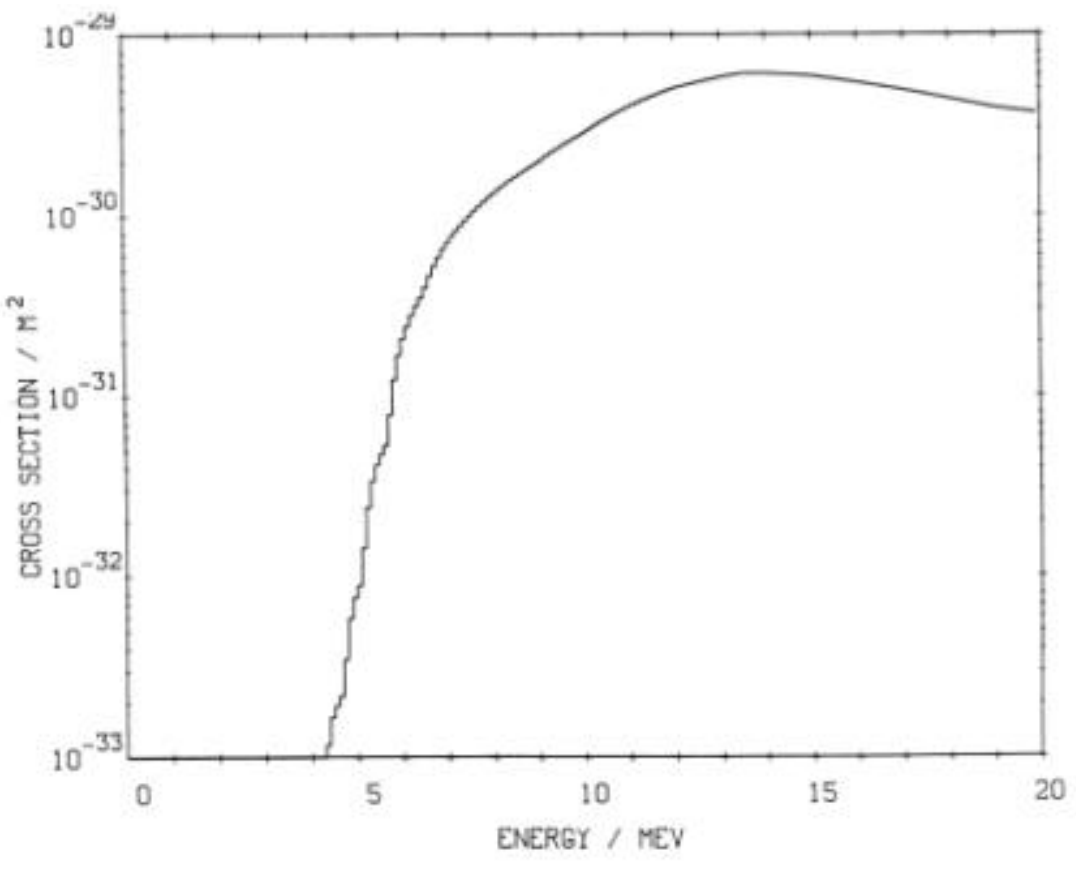

$$
\mathrm{Ti}^{48}(\mathrm{n}, \mathrm{p}) \mathrm{Sc}^{48}
$$

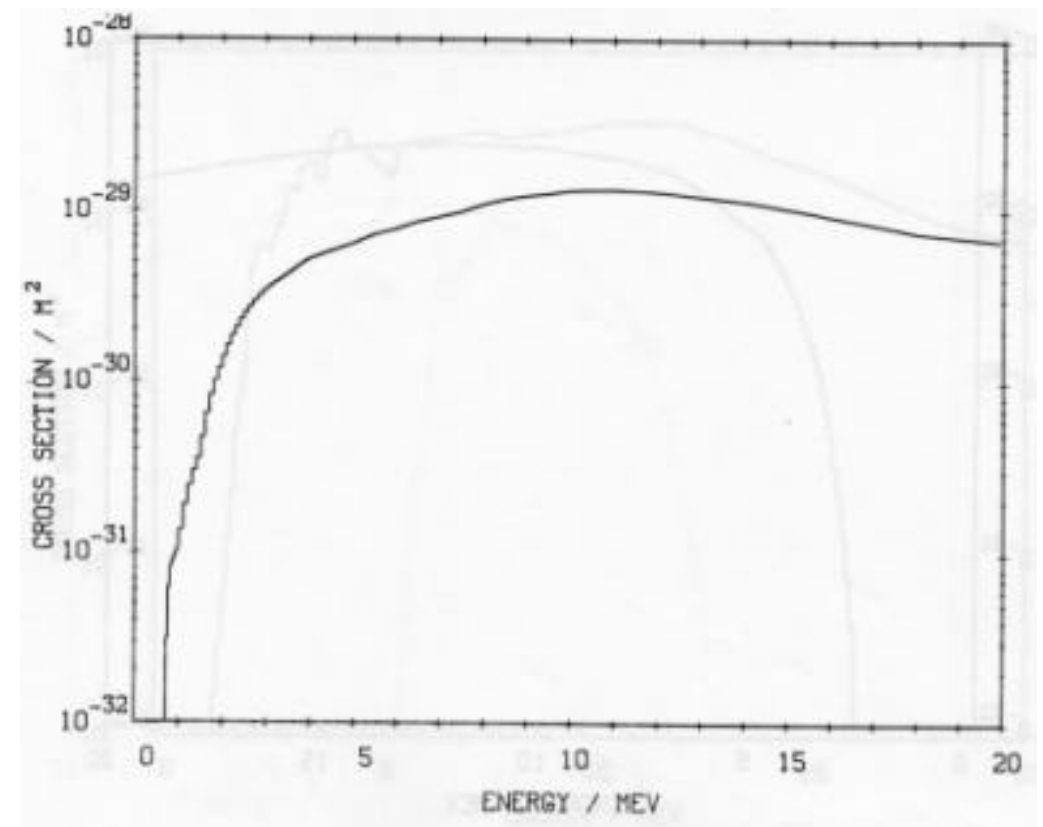

- Fis. 14 conss section arve raR ne ronction TI47P $2228 \quad 3 \quad 103$

$$
\mathrm{Ti}^{\mathbf{4 7}}(\mathrm{n}, \mathrm{p}) \mathrm{Sc}^{47}
$$




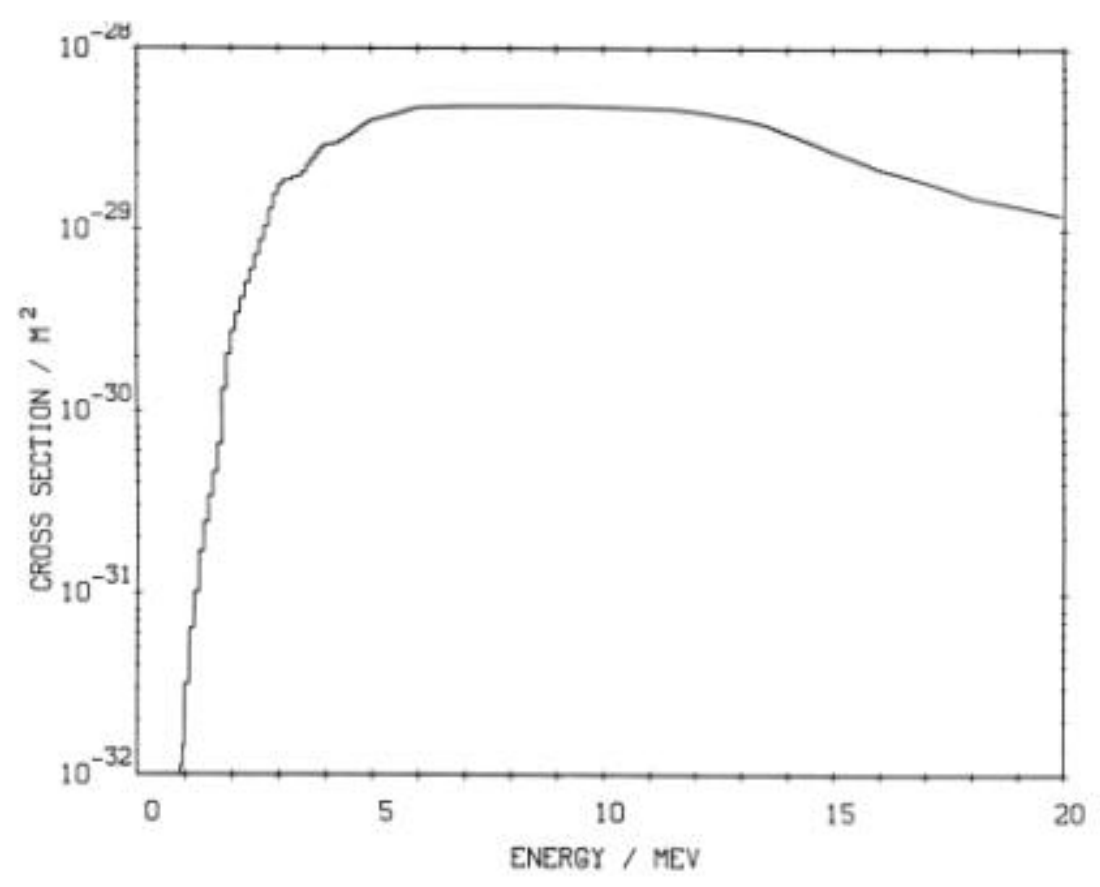

$\mathrm{Ni}^{58}(\mathrm{n}, \mathrm{p}) \mathrm{Co}^{58}$

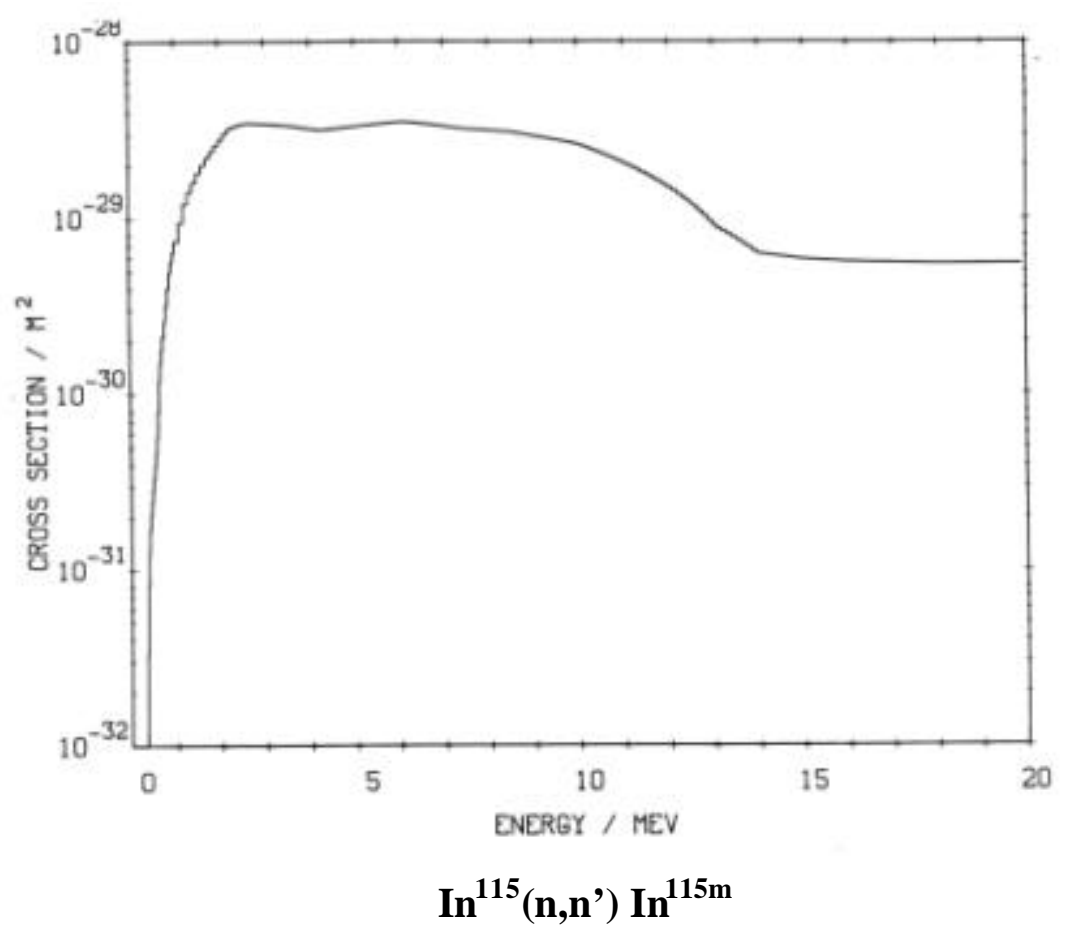

Detectores Térmicos e Epitérmicos 


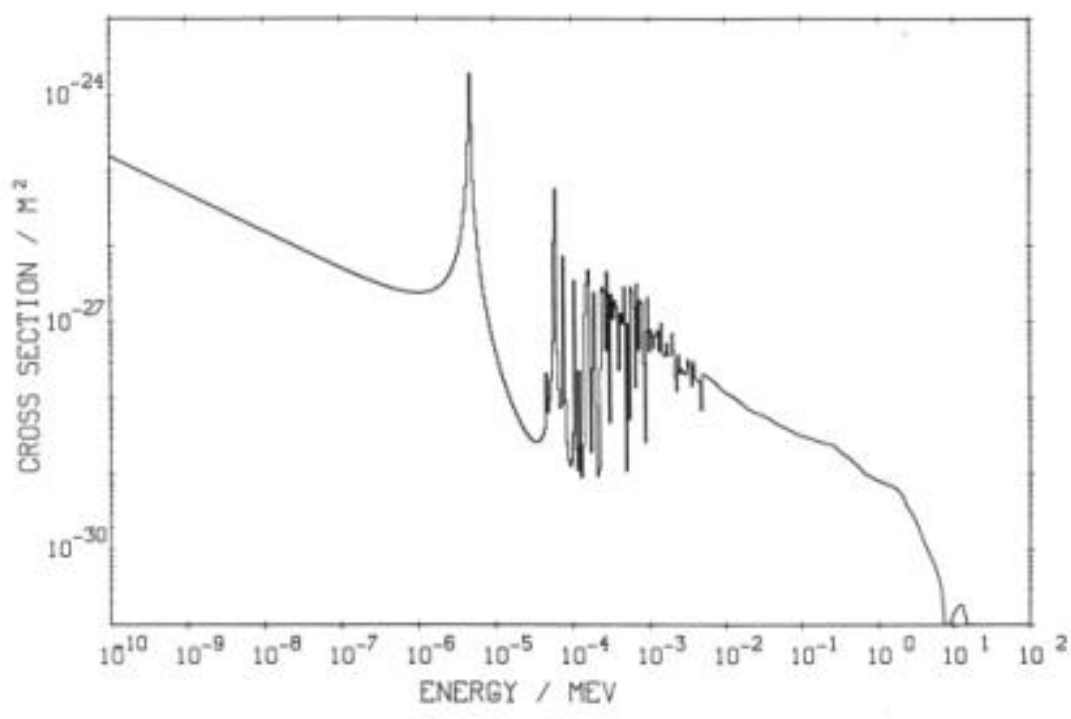

$$
\mathbf{A u}^{197}(\mathrm{n}, \boldsymbol{\gamma}) \mathbf{A u}^{198}
$$

(Detector Térmico e Epitérmico)

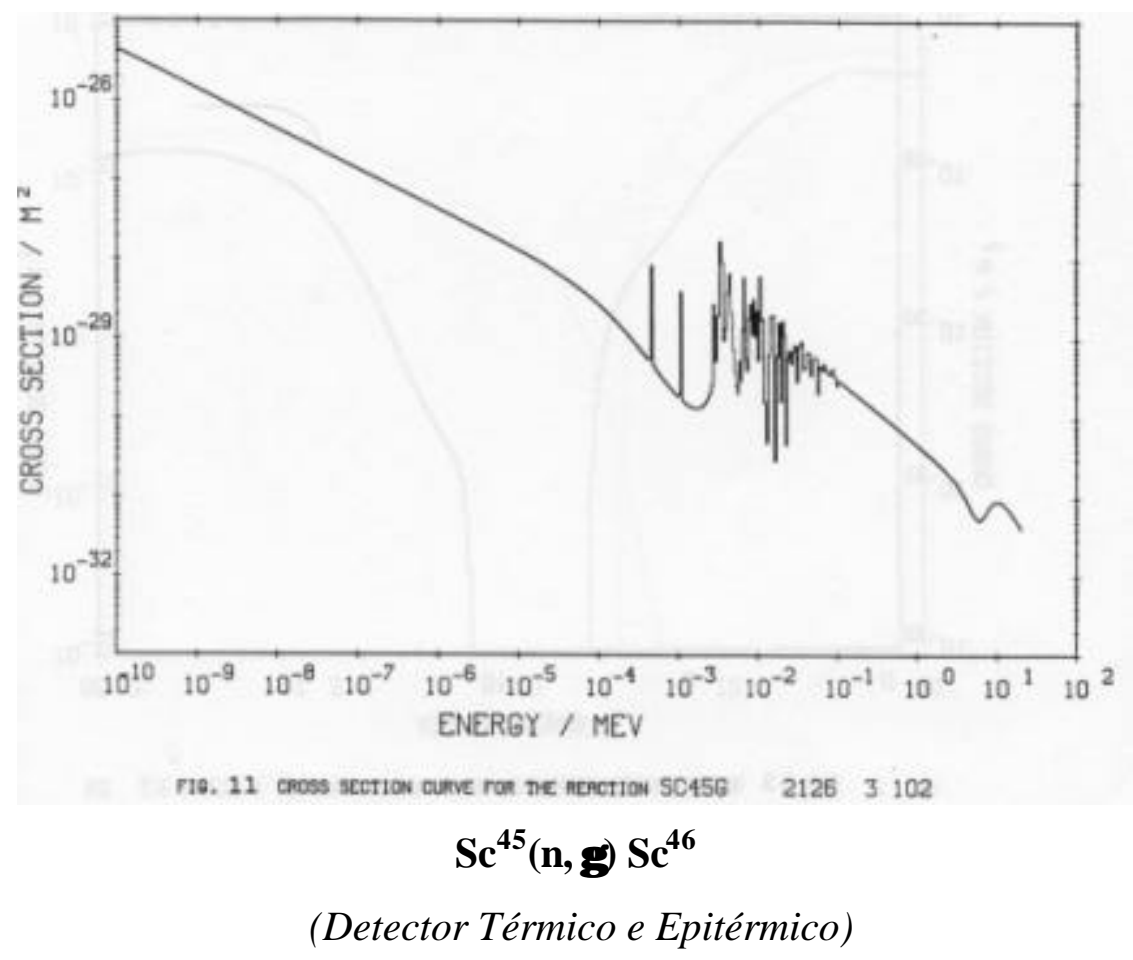




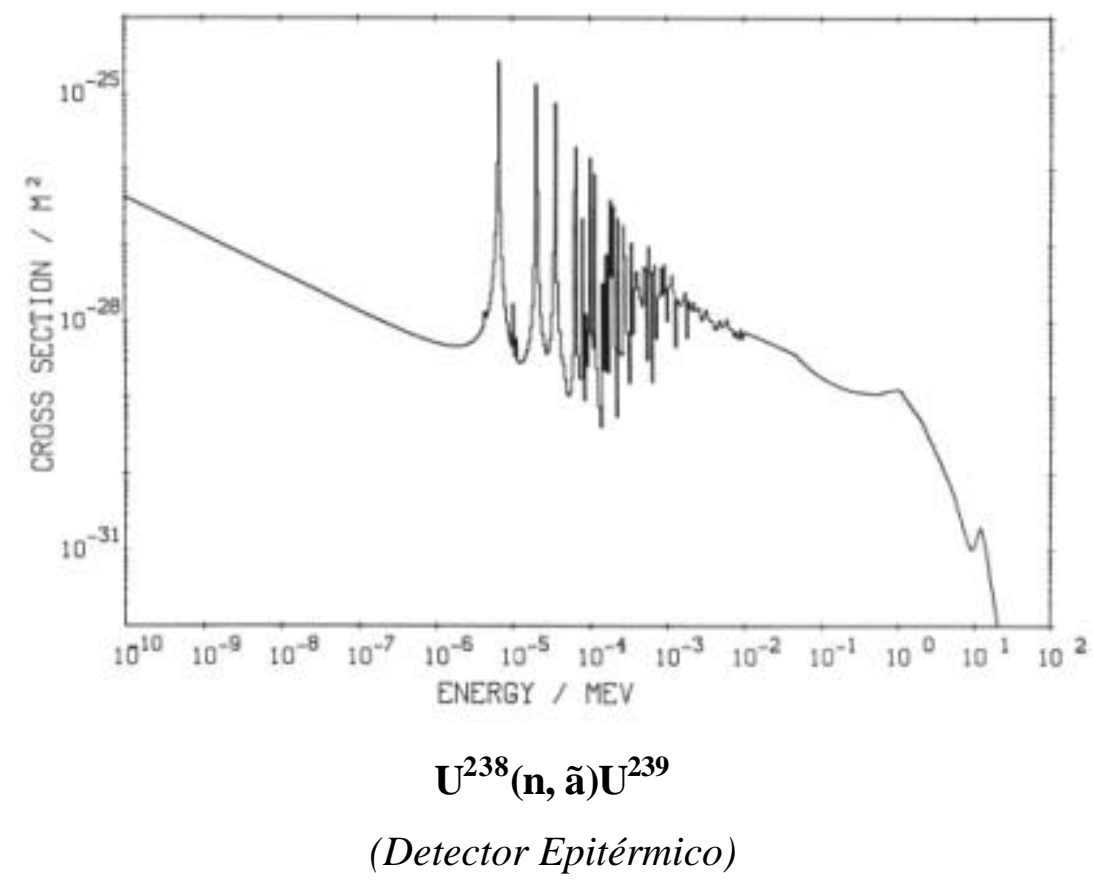

\section{ANEXO F - CÁLCULO DO FATOR DE AUTO-BLINDAGEM PARA A FOLHA DE OURO NUA E COBERTA COM CÁDMIO PELO MÉTODO DE MONTE- CARLO}

Neste anexo são exibidos os dados de entrada utilizados para a obtenção do fator de auto-blindagem pelo método de Monte-Carlo, para as folhas de ouro nua e coberta com cádmio; o cálculo foi realizado utilizando-se o código MCNP-4C. É exibida ainda neste anexo, tabela contendo os fatores de auto-blindagem obtidos pelo método de Monte-Carlo para os detectores térmicos e intermediários.

Entrada Utilizada para folha de ouro nua

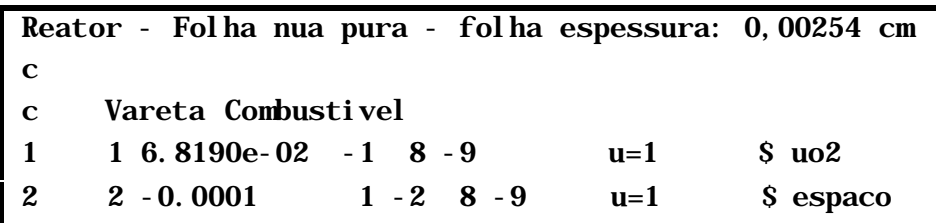




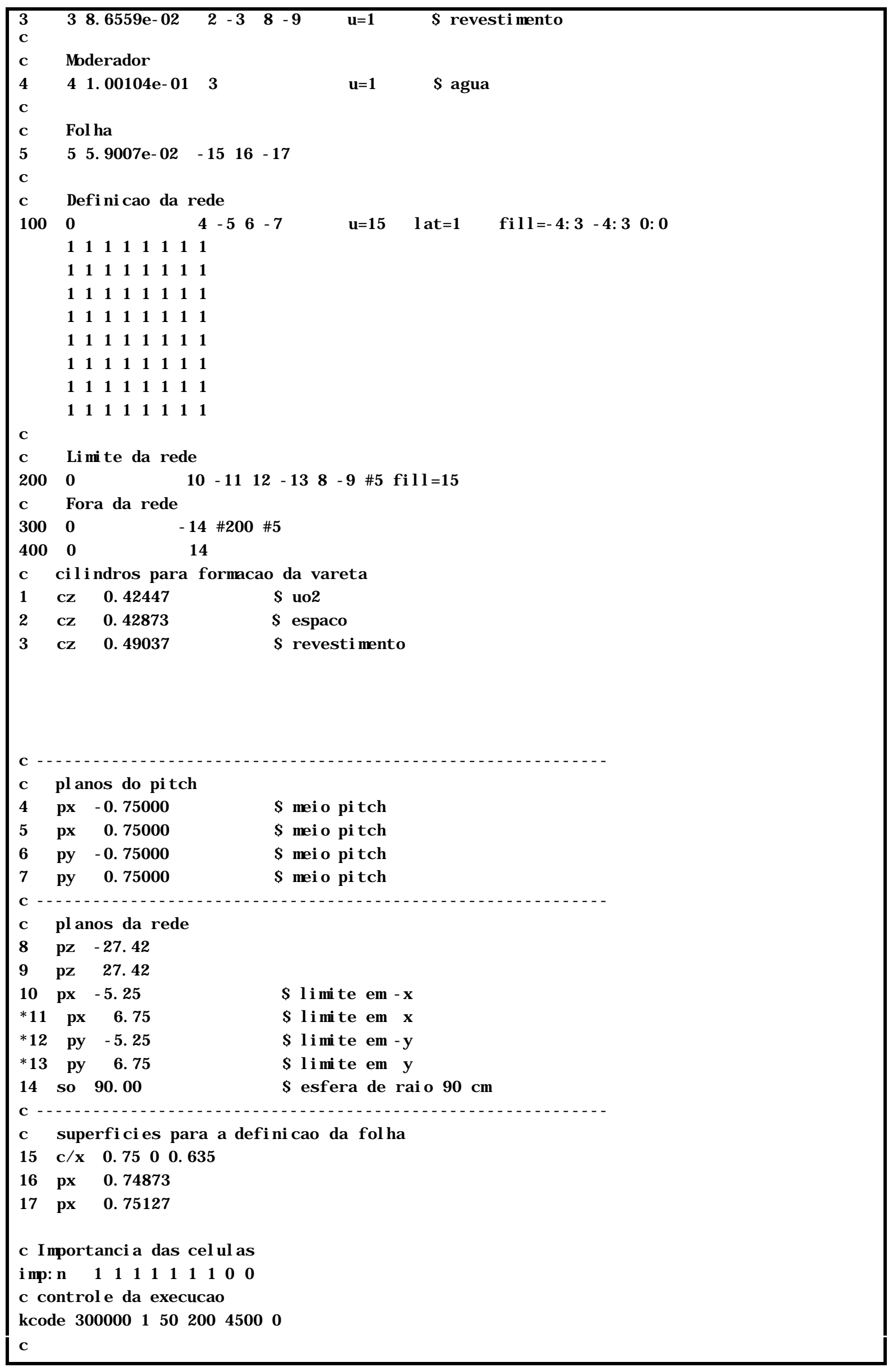


ksrc $0 \begin{array}{lll}0 & 0 & 0\end{array}$

c defi ni cao do material

c uo2

m1 92235.60c 9. 99240e- 04 92238.60c 2. 16940e- 02

8016. 60c 4. 54890e- 02 92234. 60c 7. 84620e- 06

c oxi geni o

m2 8016.60c 0.0001

c met al do encami samento

mB 26056. 60c 5. 90020e- 02 28060. 60c 8. 16250e- 03

24052. 60c 1. 68240e- 02 25055. 60c 1. 46450e- 03

14000. 60c 6. 79340e- 04 15031. 60c 4. 00400e- 05

6000. 60c 1.12390e- 04 16000. 60c $1.56170 \mathrm{e}-05$

27059. 60c 1. 74020e- 04 42000. 60c 8. 45200e- 05

C agua

$\mathrm{m} 41001.60 \mathrm{c}$ 6. 67360e- 02 8016. 60c 3. 33680e- 02

mt 4 I utr. 01t

c ouro

n5 79197. 60c 5. 9007e- 02

C

$c$ defini cao de tally

f $4: \mathrm{n} \quad 5$

f m4: n 5. 9007e- 025102

\section{Entrada utilizada para a folha de ouro coberta com cádmio}

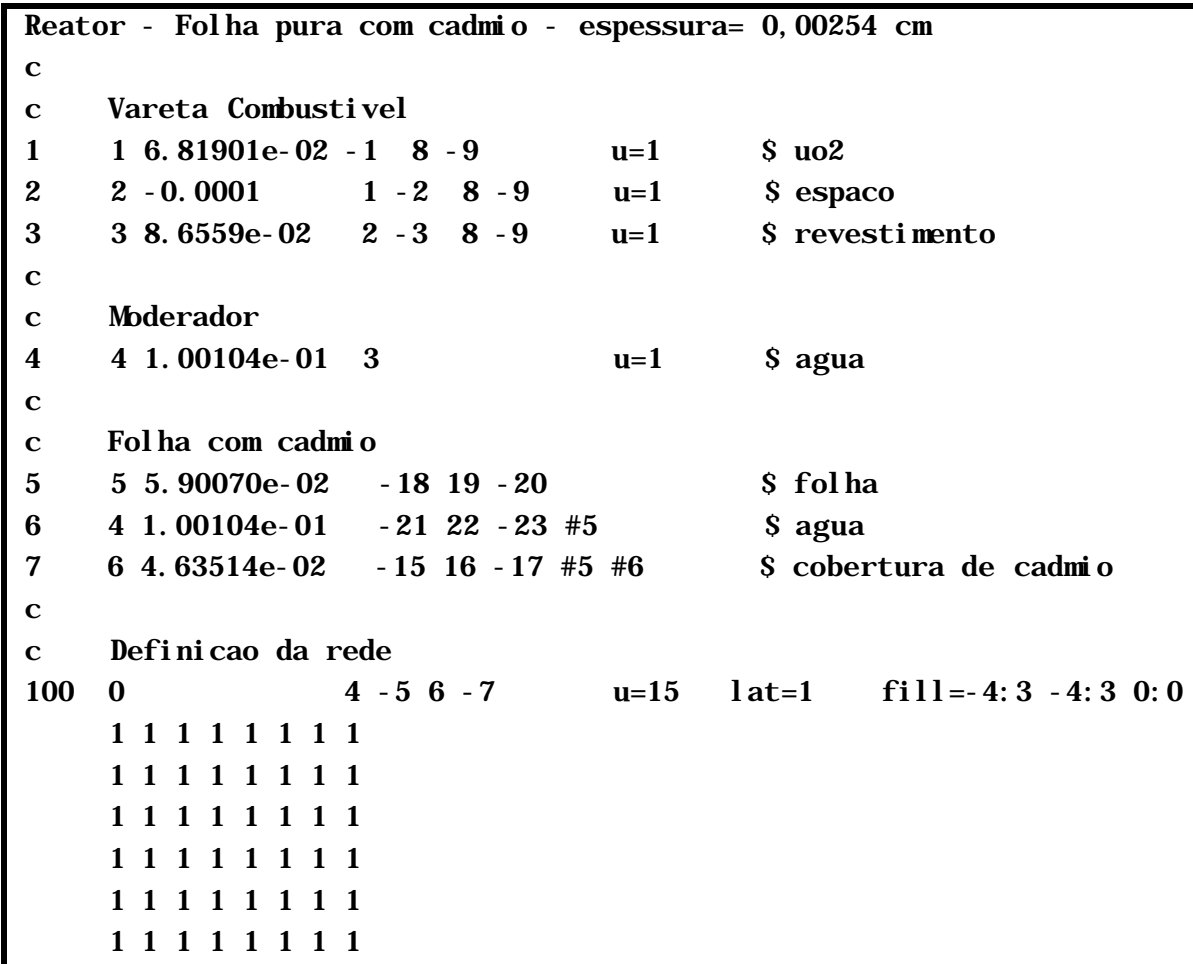




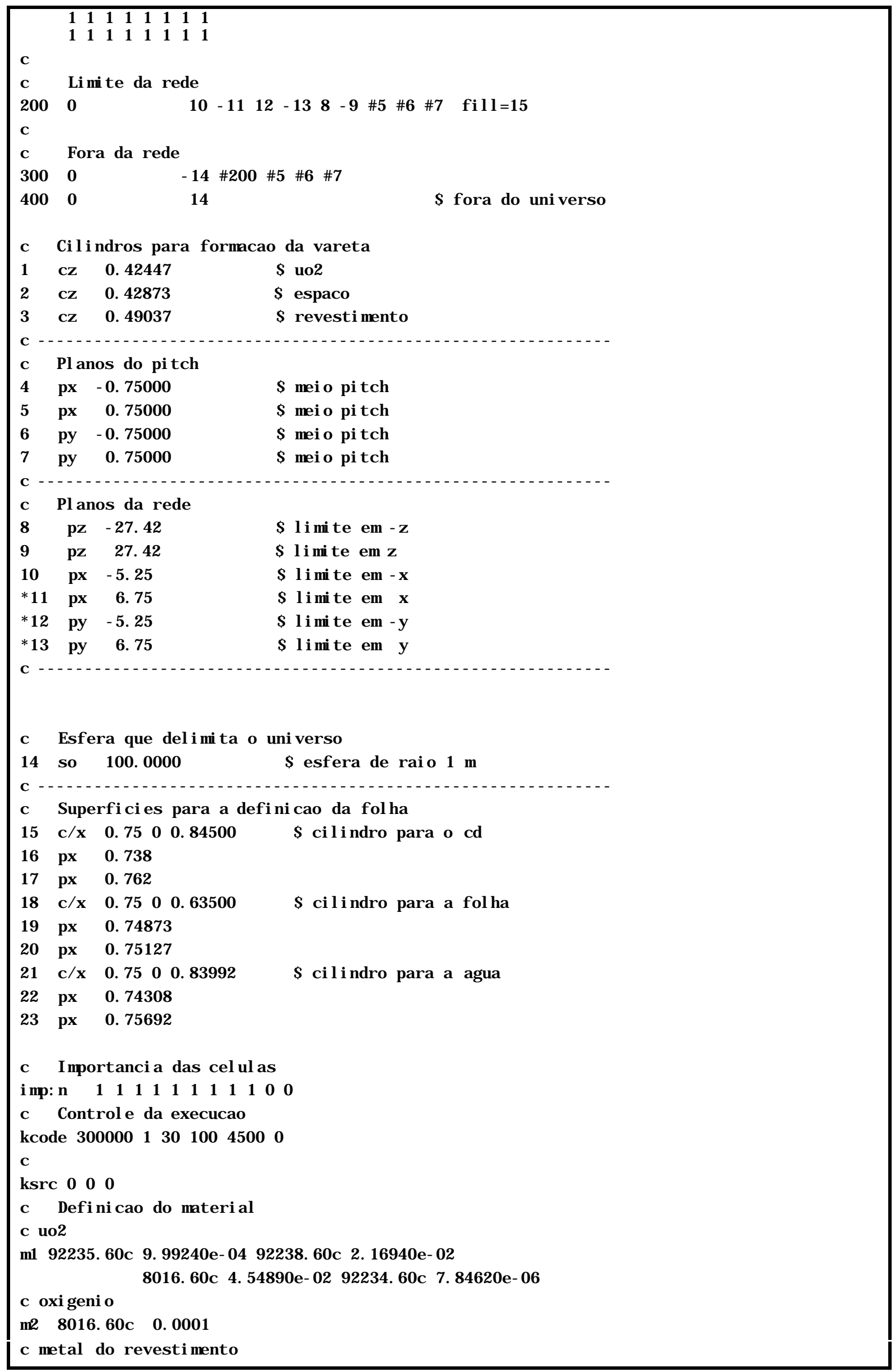




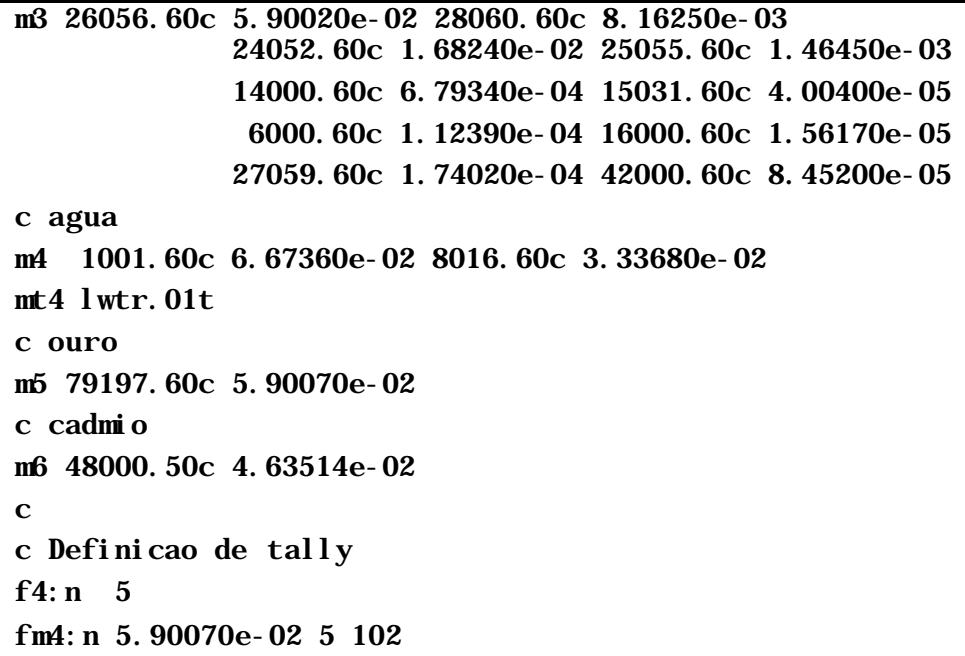

Tabela F1 - Fator de Auto-Blindagem dos detectores térmicos e intermediários

\begin{tabular}{|c|c|}
\hline Detector & Fator de Auto-Blindagem \\
\hline $\mathrm{Au}$ & 0,5160 \\
\hline $\mathrm{Au} \mathrm{c} / \mathrm{cd}$ & 0,3643 \\
\hline $\mathrm{Sc}$ & 0,9654 \\
\hline $\mathrm{Sc} \mathrm{c} / \mathrm{cd}$ & 0,9836 \\
\hline $\mathrm{U}$ & 0,3776 \\
\hline $\mathrm{Uc} / \mathrm{cd}$ & 0,4089 \\
\hline
\end{tabular}

Para os detectores de limiar que apresentam valores de seção de choque diminutos em relação aos detectores térmicos e intermediários (ordem de milibarns), as correções de auto-blindagem se mostraram desprezíveis (valores unitários), razão pela qual não são apresentadas na tabela F1. 
Referências

1. BENSCH, F. Flux depression and the absolute measurement of the thermal neutron flux density. Atomkernergie, 25 (4):257-63, 1975.

2. BERG, S. Modification of SAND II. Richland, WA, Battelle Pacific Northwest Labs., 1968. p.1-29. (BNWL-855).

3. BITELLI, U. d'U. Medida e Cálculo da Distribuição Espacial e Energéticas de Nêutrons no Núcleo do Reator IEA-R1. São Paulo : 1988. Dissertação(Mestrado)- Instituto de Pesquisas Energéticas e Nucleares.

4. BITELLI, U. d’U. Medida de Parâmetros Integrais no Reator IPEN/MB-01. São Paulo : 2001. Tese (Doutorado)- Instituto de Pesquisas Energéticas e Nucleares.

5. BITELl, T. Física e Dosimetria das Radiações. $2^{\mathrm{a}}$ Edição, Ed. Atheneu, 2006. 
6. BONDARENKO, I.I. Group Constants for Nuclear Reactor Calculation. Consultants Bureau, New York, NY, 1964.

7. BRENNER, D.J. Monte carlo self-shielding corrections for use with neutron spectrum unfolding codes. Los Alamos, N.M., Los Alamos National Laboratory, 1981. p-175-7. (Technical notes).

8. BRIEMEISTER, J.F; A General Monte Carlo N-Particle Transport Code (Version -4C), Los Alamos National Laboratory, LA-13709-M, 2000.

9. DERCKX, R. Unfolding methods in neutron spectra measurements by foil activation technique. Nucl. Instrum. Methods, 105:1-4, 1972.

10. DUDERSTADT, J.J.; HAMILTON, L.J. Nuclear Reactor Analysis. New York, John Wiley \& Sons, 1976.

11. FISCHER, A., TORI, L.: The RFSP programme for unfolding neutron spectra from activation data. Report INDC (HUN) - 8/U. International atomic energy agency, Vienna, 1972.

12. FISCHER, A.: RFSP-JL. A computer programme for unfolding neutron spectra from activation data. Report F J, December, 1977.

13. GLOWER, D.D. Experimental reactor analysis and radiation measurements. New York, McGraw-Hill, 1965.

14. GONÇALVES, I.F.; MARTINHO, E.; SALGADO, J. Monte Carlo Calculation of ephitermal nêutron resonance self-shielding factors in foils of different materials. Instituto Tecnológico e Nuclear. Agosto, 2001.

15. GONÇALVES, I.M.C.; Sobre a determinação de Espectros de Neutrões Rapidos num Reactor Nuclear. Aplicação ao Reactor Português de Investigação. Instituto de Ciências e Engenhaia Nucleares. Outubro, 1986. 
16. GRADER, R.J. Measuring reactor neutron spectra with threshold detectors. Rev. Sci. Instrum., 32(8):933,1961 .

17. GREER, C. L., WLKER, J.V: A procedure for the computation of neutron flux from foil activation data. Spectra code, contribution to radiation measurements in nuclear power, proceedings of international conference at Berkeley nuclear laboratories, page 270 (Institute of Physics and Physical Society, London, 1966).

18. GREER, C. L., HALBLEIB, J. A., WALKER, J. V.: A technique for unfolding neutron spectra from activation measurements, Report SC-RR-67-746-1967.

19. INTERNATIONAL ATOMIC ENERGY AGENCY. Neutron cross section for reactor dosimetry. Proceedings of a meeting on..., held in Vienna, 15-19 Nov., 1976. Vienna, 1978. v.1.

20. INTERNATIONAL ATOMIC ENERGY AGENCY . Nêutron Fluence Measuremt Vienna, 1970. (Technical Reports Series $\left.n^{\circ} 107\right)$.

21. KAM, F. B. K, STALLMANN, F. W.: Crystal Ball. A computer code for determining neutron spectra from activation measurements. Report ORNL/TM - 4601. OAK Ridge National Laboratory, June, 1974.

22. KNOLL, G.F. Radiation detection and measurements. New York, Wiley, 1979.

23. KONDO, I. \& SAKURAI,K. Experimental evaluation of reactor neutron spectrum in irradiation field. J. Nucl. Sci Technol., 18(6):461-72, 1981.

24. LAMARSH, J.R. Introduction to nuclear theory. Reading, Mass., AddisonWesley, 1966.

25. LUX, I. Calculation of Foil-Activation-Correction by Monte Carlo Method. Central Research Institute for Physics, Budapest, Hungary, KFKI-1981-38, 1981.

26. MARTINHO, E., GONÇALVES, I.F., SALGADO, J. Universal Curve of Epithermal Neutron Resonance Self-Shielding Factors in Foils, Wires and Spheres. Instituto Tecnológico e Nuclear, Sacavém 2686-953, Portugal, Outubro 2002. 
27. McELROY. W.N, et al. : SAND . Neutron Flux Spectra Determinations by multiple foil Activation $n$ iterative e method. Oak Ridge National Laboratory, RSIC Computer Code Collection CCC - 112. May 1969.

28. McELROY, W.N.; BERG, S.; CROCKETT, T.; HAWKINS, R.G. SAND II - $\boldsymbol{a}$ computer-automated interactive method for neutron flux-spectra determination by foil activation. Kirtland AFB, NM, Air Force Weapons Laboratory, Sept. 1967. (AFWL-TR-67-41).

29. MESTNIK F, J. A distribuição especial do fluxo de nêutrons no núcleo do reator IEA-R1, obtida por meio da ativação de folhas. São Paulo, 1978. (Dissertação de mestrado, Escola Politécnica, Universidade de São Paulo). (IEA-DT-132).

30. MO, S.C \& OTT, K.O. Resonance self shielding corrections for detector foils in fast neutron spectra. Nucl. Sci. Eng., 95:214-24, 1987.

31. NORDHEIN, L.W. KUNIUR, G.F. A program of Research and Calculation of Resonance Absorption. San Diego, CA, Gulf General Atomic CO., 1961.

32. PEREY, F. G.: Least - Square dosimetry unfolding: The program STAY'SL, Report ORNL/TM-6062, ENDF-254, OAK Ridge, March, 1986.

33. PROFIO, A.E. Experimental reactor physics. New York, Wiley, 1976.

34. RANT, J. Measurements of neutron flux distributions by activation detectors. In: INTERNATIONAL ATOMIC ENERGY AGENCY. Aplication of small computers to research reactor operation: international training course, Ljubljana, 126 Jun., 1987.

35. REUS, U.; WESTMEIER, W. Atomic Data and Nuclear Data Tables. Marburg, Academic Press, 1983.

36. RITCHIE, R.H. \& ELDRIDGE, H.B. Thermal neutron flux depression by absorbing foils. Nucl. Sci. Eng., 8:300-11, 1960. 
37. SUICH, J.E, HONECK, H.C. The Hammer System Heterogeneous Analysis of MiltGrroup Methods of Exponential and Reactor. Aiken, S.C., Du Pont de Nemours, Savannah River Laboratory, 1967.

38. SZONDI. E.J, ZSOLNAY. E.M : Documentation of the Code SANDBP. Report, Institute of Nuclear Techniques, Technical University of Budapest, Budapest, September 1992.

39. TASHANI, H. A. Neutron Spectrum Calculation and Measurement at the Nuclear Reactor of Budapest Technical University. Budapeste 1997. Doctor of Philosophy Dissertation.

40. TSOULFANIDIS, N.; EDWARDS, D.R.; KAO, L.; YIN, F. Neutron fluence and threshold foil reaction rates in PWRs. Trans. Am. Nucl. Soc., 45:592-3, 1983.

41. WATT, B.E. Energy Spectrum of neutrons from thermal fission of U235, Phys. Rev. 87 (1952). 1037.

42. WESCOTT, C.M. Effective cross section values for well-moderated thermal reactor spectra. Chalk River, Ontario, Atomic Energy of Canada, Sept. 1960.

43. ZIJP, W. L, ZSOLNAY, E. M, SZONDI, E. J, NOLTHINUS, H. J, VERHAAG, G. C. H, GULLEN, D. E, ERTEK, G.: Final report on the real-80 exercise, ECN-128. Petten, February, 1982.

44. ZIJP, W. L, ZSOLNAY, E. M, SZONDI, E. J, NOLTHINUS, H. J.: Final report on the real-84 exercise, ECN-212. Petten, November, 1988.

45. ZIJP, W. L., NOLTHENIUS, H. J. Neutron self shielding of activation detectors used in spectrum unfolding. Petten, Reactor Centrum Nederland, Aug. 1975. (RCN-231).

46. ZIJP, W. L., BAARD, J. Nuclear data Guide for Reactor Neutron Metrology. ECN - 70, 1979. 
47. ZIJP, W. L. Comparison of Neutron Spectrum Unfolding Codes. International Atomic Energy Agency . IAEA - R - 1811 -F , 1979.

48. ZIJP, W. L. Review of activation methods for the determination of neutron flux density spectra.. Report, RCN-241, Petten, 1976.

49. ZSOLNAY, E.M. Neutron Flux and Spectrum Measurement by Activation Method. , Institute of Nuclear Techniques, Technical University of Budapest, Budapest, 1993.

50. ZSOLNAY, E.M. On the Quality of Uncertainty Information in the Internacional Reactor Dosimetry File IRDF 90. Netherlands Energy Research Foundation ECN, June, 1992.

51. ZSOLNAY, E.M, SZONDI, E. J.; Neutron spectrum determination by multiple foil activation method Periodica Polytechnica, Electrical Engineering 26 (1982).

52. ZSOLNAY, E.M, NOLTHENIUS, H.J., GREENWOOD, L.R., SZONDI, E. J. Reference data file for neutron spectrum adjustment and related radiation damage calculation. Proceedings of the 7th ASTM-EURATOM Symposium on Reactor Dosimetry. Strasbourg, France, 27-31 Aug., 1990. EUR 14356 EN. Eds: G. Tsotridis et al.Kluwer Academic Press (1992).

53. ZSOLNAY, E.M, SZONDI, E. J: Experiences with neutron spectrum unfolding codes in different neutron spectra. Report BME-TER-RES-3/82. Nuclear reactor of the technical University, Budapest, April 1982, ISBN 9634211666. 
